\title{
WestVirginiaUniversity
}

THE RESEARCH REPOSITORY @ WVU

Graduate Theses, Dissertations, and Problem Reports

2001

\section{Effects of baffles on damping lateral fluid sloshing oscillations in tanker trucks}

Rohit Kumar Tanugula

West Virginia University

Follow this and additional works at: https://researchrepository.wvu.edu/etd

\section{Recommended Citation}

Tanugula, Rohit Kumar, "Effects of baffles on damping lateral fluid sloshing oscillations in tanker trucks" (2001). Graduate Theses, Dissertations, and Problem Reports. 1211.

https://researchrepository.wvu.edu/etd/1211

This Thesis is protected by copyright and/or related rights. It has been brought to you by the The Research Repository @ WVU with permission from the rights-holder(s). You are free to use this Thesis in any way that is permitted by the copyright and related rights legislation that applies to your use. For other uses you must obtain permission from the rights-holder(s) directly, unless additional rights are indicated by a Creative Commons license in the record and/ or on the work itself. This Thesis has been accepted for inclusion in WVU Graduate Theses, Dissertations, and Problem Reports collection by an authorized administrator of The Research Repository @ WVU. For more information, please contact researchrepository@mail.wvu.edu. 
Effects of Baffles on Damping Lateral Fluid Sloshing Oscillations in Tanker Trucks

By

Rohit Tanugula

\author{
A THESIS \\ Submitted to the College of Engineering and Mineral Resources \\ at \\ West Virginia University \\ in partial fulfillment of the requirements \\ for the degree of \\ Master of Science \\ in \\ Mechanical Engineering \\ Mridul Gautam, Ph.D, Chair \\ Victor H. Mucino, Ph.D. \\ Kenneth H. Means, Ph.D. \\ Department of Mechanical and Aerospace Engineering \\ Morgantown, West Virginia \\ 2001
}




\section{Abstract \\ Effects of Baffles on Damping Lateral Fluid Sloshing Oscillations in Tanker Trucks Rohit Tanugula}

Partially filled heavy-duty tanker trucks maneuvering constant radius turns or lane change maneuvers have lower rollover threshold than other vehicles because of the high center of gravity and dynamic sloshing of the liquid payload. Payloads are often toxic and hence, rollover accidents can be catastrophic. Therefore, attempts to increase the threshold velocity and predicting its value under various on-road maneuvers are of paramount importance.

This research was aimed at studying the effects of baffles on reducing the lateral sloshing and thereby, improving the stability of heavy-duty trucks. Various configurations (horizontal, vertical, horizontal and vertical, and diagonal) of baffles were considered for this purpose. The objective of this project was to determine the best configuration of baffles for various shapes of tank-containers, percent fill levels, and different kinds of maneuvers.

Damping effects of the kinematic viscosity of the fluid inside the tank were also studied in this research. This was done to determine the trammel pendulum parameters for pendulum-fluid dynamic effect matching. Trammel pendulums can also be used to study and simulate the lateral fluid sloshing effects in partially filled tankers.

Kinematic viscosity of the fluid was found to have no significant damping effect on the lateral fluid sloshing. Conclusions were drawn regarding the effects of various baffles. Damping provided at the free surface of the fluid was found to have the best effect. Horizontal and diagonal baffles were found to be the best for constant radius turns with a maximum reduction of $60 \%$ in the moments. In a TOP lane change maneuver, 
diagonal baffles were found more effective for lower fill levels up to $50 \%$. For fill levels higher than $50 \%$ vertical baffles were found to be the best configuration. 


\section{ACKNOWLEDGEMENTS}

I would like to thank my advisor, Dr. Mridul Gautam, for his continuous support, advice, and guidance throughout the last two years. He was always there whenever I needed him. Working under his supervision has been a wonderful experience.

I would also like to thank Dr. Victor Mucino for his support and guidance anytime I needed. His continued interest and invaluable suggestions have been a constant inspiration and source of encouragement. Thanks are also due to Dr. Kenneth Means for participating on my committee.

I would also like to credit Sandeep Mehta, Eric Saunders, Mohamed Salem and Leo Marbun for their support and inspiration. Sandeep has taught me many things and has been of great help. Whenever I was stuck with a problem he was there to help me out with various options and countless hours of discussions.

I would also like to thank my parents, brothers and friends for the love and motivation they have provided throughout my studies. 


\section{TABLE OF CONTENTS}

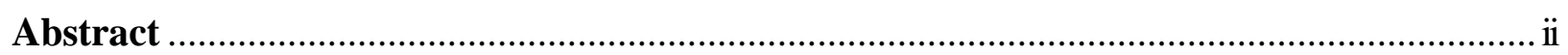

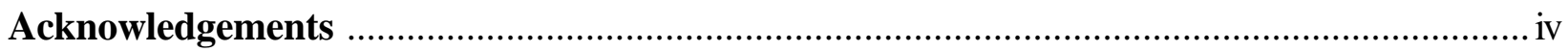

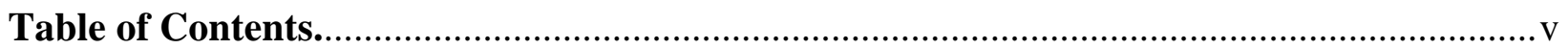

List of Figures................................................................................................................ vii

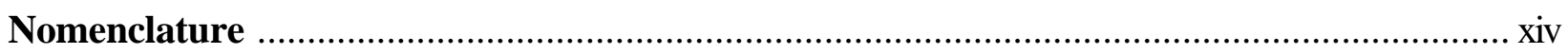

Chapter 1. Introduction and Problem Statement .............................................................

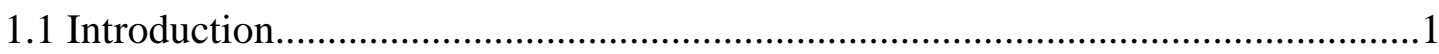

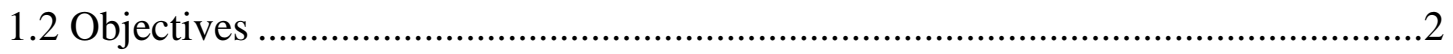

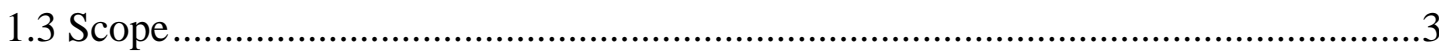

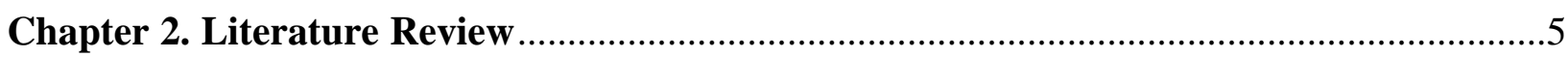

Chapter 3. Model Description and Verification...................................................................17

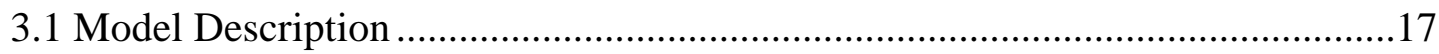

3.2 Building the Case File .....................................................................................18

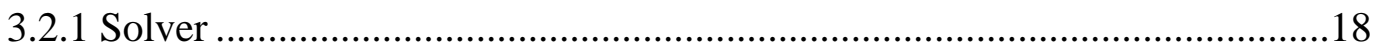

3.2.2 Physical Model.........................................................................................

3.2.3 Volume of Fluid Model ........................................................................20

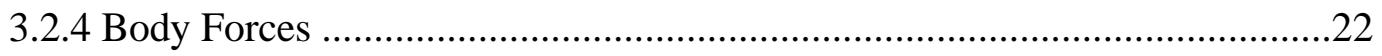

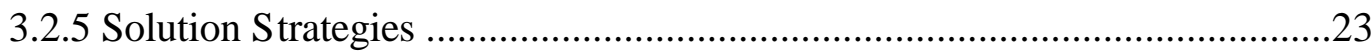

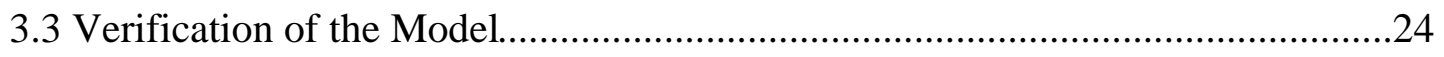

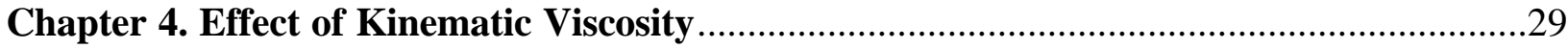

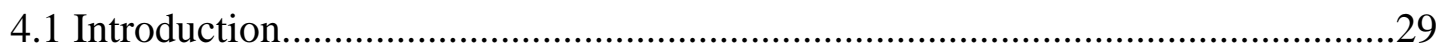

4.2 Pendulum-Fluid Dynamic Effect Matching ............................................................30 


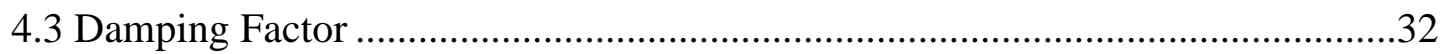

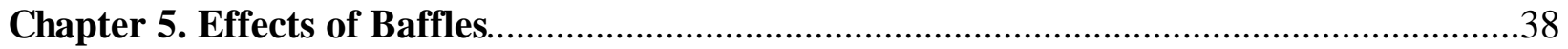

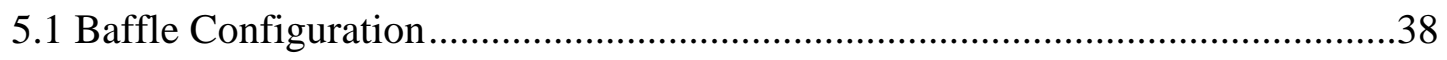

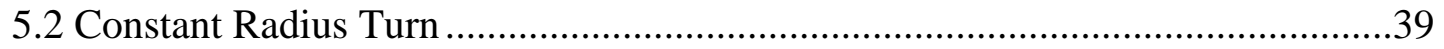

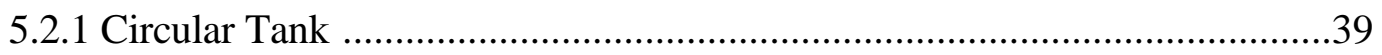

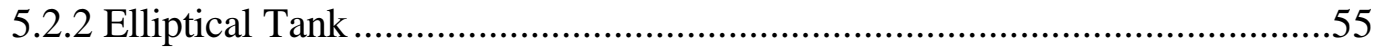

5.3 TOP Lane Change Maneuver......................................................................65

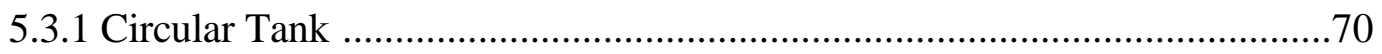

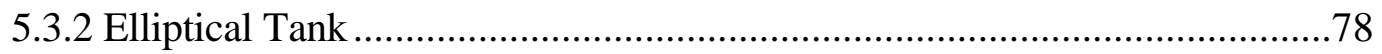

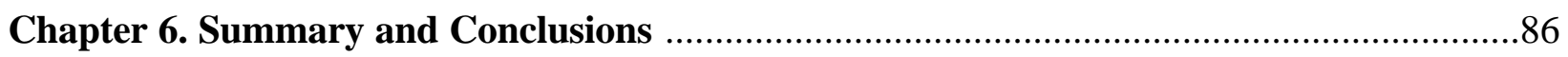

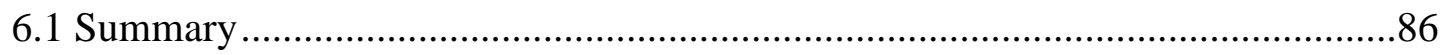

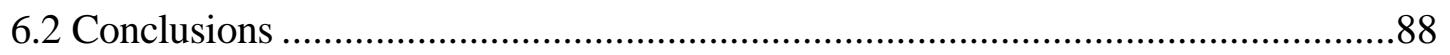

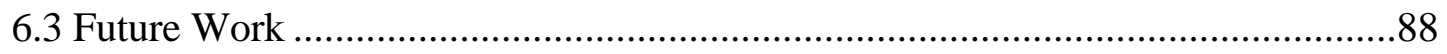

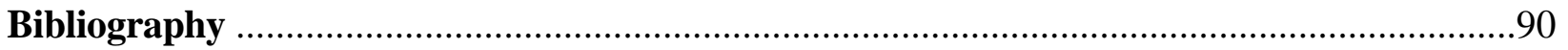

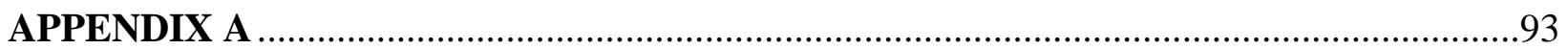

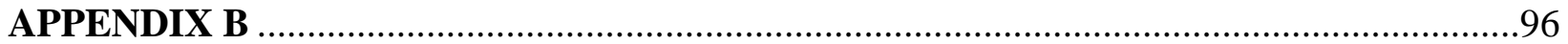




\section{LIST OF FIGURES}

Figure 1. Variation of damping factor with kinematic viscosity of water for a fixed ring baffle mounted in 30 - inch diameter tank [3] f................................................................

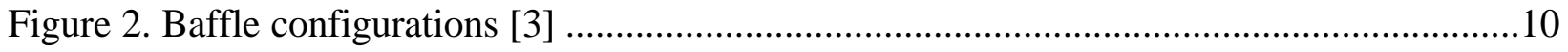

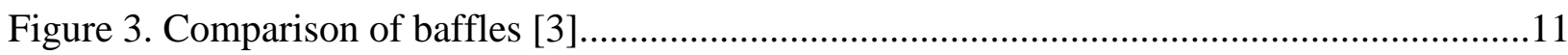

Figure 4. Overturning limits for steady state cornering [13] .............................................12

Figure 5. Ratio of steady state overturning moment of an un-compartmented to

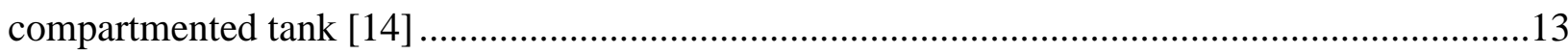

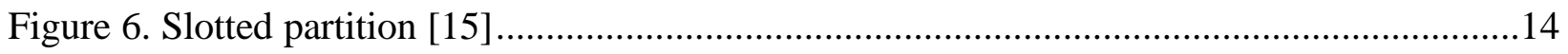

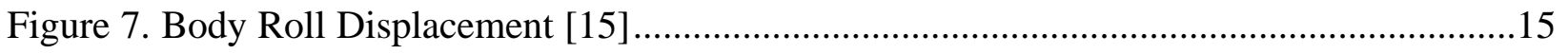

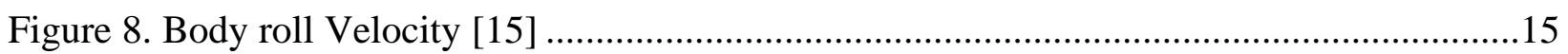

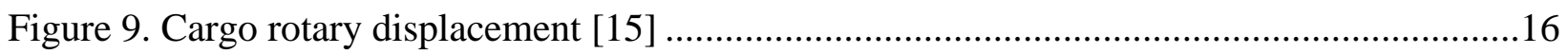

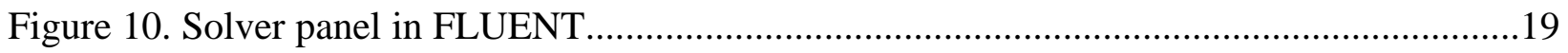

Figure 11. Multiphase model panel in FLUENT .............................................................21

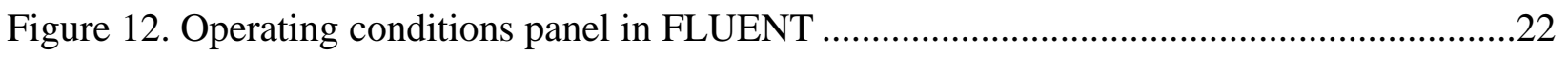

Figure 13. Volume fraction of water in a circular tank with $30 \%$ fill level............................23

Figure 14. Solution controls panel in FLUENT ...........................................................24

Figure 15. Horizontal force coefficients for different models ..............................................27

Figure 16. Moment coefficients for different models .......................................................27

Figure 17. Frequency parameter "K" for different models .................................................28

Figure 18. Center of gravity of fluid volume contour drawn by an elliptical trammel

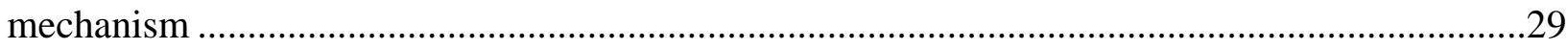


Figure 19. Damping factor (horizontal force) in a circular tank with lateral acceleration of $0.3 \mathrm{~g}$ .33

Figure 20. Damping factor (moment) in a circular tank with lateral acceleration of $0.3 \mathrm{~g}$. 34

Figure 21. Damping factor (horizontal force) in an elliptical tank with lateral acceleration of $0.3 \mathrm{~g}$ .34

Figure 22. Comparison of damping factor for different fluids with those of same fluid with only the kinematic viscosity changed .36

Figure 23. Configuration of various baffles considered ..................................................38

Figure 24. Moments and forces on the tank walls 40 Figure 25. Peak moments for circular tank with horizontal baffles and lateral acceleration of $0.3 \mathrm{~g}$ .41

Figure 26. $\mathrm{C}_{\mathrm{m}}$ for circular tank with horizontal baffles and lateral acceleration of $0.3 \mathrm{~g}$ 41

Figure $27 . \mathrm{C}_{\mathrm{f}}$ for circular tank with horizontal baffles and lateral acceleration of $0.3 \mathrm{~g}$.

Figure 28. Percentage ratios of peak moments in a circular tank with horizontal baffles to peak moments in a circular tank without any baffles.

Figure 29. Volume fraction of fluid in a $30 \%$ fill tank with horizontal baffles of $1 / \mathrm{a}$ ratio $=0.5$ at 0.8 seconds

Figure 30. Volume fraction of fluid in a $70 \%$ fill tank with horizontal baffles of $1 / \mathrm{a}$ ratio $=0.5$ at 0.8 seconds

Figure 31. Peak moments for circular tank with vertical baffles and lateral acceleration of $0.3 \mathrm{~g}$ .45

Figure $32 . \mathrm{C}_{\mathrm{m}}$ for circular tank with vertical baffles and lateral acceleration of $0.3 \mathrm{~g}$. .45 
Figure $33 . \mathrm{C}_{\mathrm{f}}$ for circular tank with vertical baffles and lateral acceleration of $0.3 \mathrm{~g}$.

Figure 34. Percentage ratios of peak moments in a circular tank with vertical baffles to peak moments in a circular tank without any baffles

Figure 35. Peak moments for circular tank with lateral acceleration of $0.3 \mathrm{~g}$ and

horizontal-vertical baffles

Figure $36 . \mathrm{C}_{\mathrm{m}}$ for circular tank with lateral acceleration of $0.3 \mathrm{~g}$ and horizontal-vertical

baffles

Figure $37 . \mathrm{C}_{\mathrm{f}}$ for circular tank with lateral acceleration of $0.3 \mathrm{~g}$ and horizontal-vertical

baffles

Figure 38. Peak moments for circular tank with diagonal baffles and lateral acceleration

of $0.3 \mathrm{~g}$ .50

Figure $39 . \mathrm{C}_{\mathrm{m}}$ for circular tank with lateral acceleration=0.3g and diagonal baffles .50

Figure $40 . \mathrm{C}_{\mathrm{f}}$ for circular tank with lateral acceleration of $0.3 \mathrm{~g}$ and diagonal baffles .51

Figure 41. Percentage ratios of peak moments in a circular tank with horizontal-vertical baffles to peak moments in a circular tank without any baffles .51

Figure 42. Percentage ratios of peak moments in a circular tank with diagonal baffles to

peak moments in a circular tank without any baffles .52

Figure 43. Peak moments for various baffles with $1 / \mathrm{a}=0.75$ in a circular tank through

constant radius turn. .53

Figure 44. Moment with time for various baffles in a circular tank .53

Figure 45. Peak moments for elliptical tank with lateral acceleration of $0.3 \mathrm{~g}$ and

horizontal baffles .56 
Figure $46 . \mathrm{C}_{\mathrm{m}}$ for elliptical tank with lateral acceleration of $0.3 \mathrm{~g}$ and horizontal baffles .56

Figure 47. $\mathrm{C}_{\mathrm{f}}$ for circular tank with lateral acceleration of $0.3 \mathrm{~g}$ and horizontal baffles .57

Figure 48. Percentage ratios of peak moments in an elliptical tank with horizontal baffles to peak moments without any baffles .57 Figure 49. Peak moments for elliptical tank with lateral acceleration of $0.3 \mathrm{~g}$ and vertical baffles. .58

Figure $50 . \mathrm{C}_{\mathrm{m}}$ for elliptical tank with lateral acceleration of $0.3 \mathrm{~g}$ and vertical baffles .58

Figure $51 . \mathrm{C}_{\mathrm{f}}$ for elliptical tank with lateral acceleration of $0.3 \mathrm{~g}$ and vertical baffles

Figure 52. Percentage ratios of peak moments in an elliptical tank with vertical baffles to peak moments without any baffles

Figure 53. Peak moments for elliptical tank with lateral acceleration of $0.3 \mathrm{~g}$ and horizontal-vertical baffles 61

Figure $54 . \mathrm{C}_{\mathrm{m}}$ for elliptical tank with lateral acceleration of $0.3 \mathrm{~g}$ and horizontal-vertical baffles .61

Figure $55 . \mathrm{C}_{\mathrm{f}}$ for elliptical tank with lateral acceleration of $0.3 \mathrm{~g}$ and horizontal-vertical baffles 62

Figure 56. Percentage ratios of peak moments in an elliptical tank with horizontal-vertical baffles to peak moments without any baffles 62

Figure 57. Peak moments for elliptical tank with lateral acceleration of $0.3 \mathrm{~g}$ and diagonal baffles. .63

Figure 58. $\mathrm{C}_{\mathrm{m}}$ for elliptical tank with lateral acceleration of $0.3 \mathrm{~g}$ and diagonal baffles .63 
Figure $59 . \mathrm{C}_{\mathrm{f}}$ for elliptical tank with lateral acceleration of $0.3 \mathrm{~g}$ and diagonal baffles

Figure 60. Peak moments for various baffles with $1 / \mathrm{a}=0.75$ in an elliptical tank through constant radius turn .65

Figure 61. Path of the test vehicle during the TOP Lane Change Test.....................................66

Figure 62. Path of the test vehicle during the NATO Lane Change Test .................................66

Figure 63. Calculated lateral acceleration for the trailer under study, at 50\% full

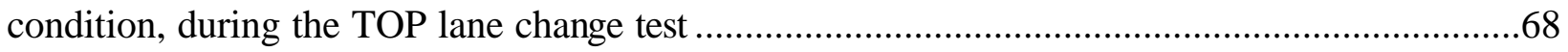

Figure 64. Comparison of actual data and reduced data ...................................................69

Figure 65. Peak moments for circular tank with horizontal baffles in a TOP lane change

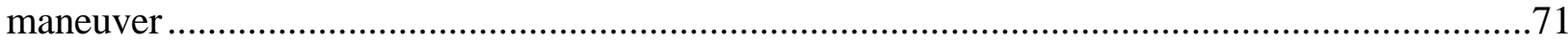

Figure $66 . \mathrm{C}_{\mathrm{m}}$ for circular tank with horizontal baffles in a TOP lane change maneuver...........71

Figure $67 . \mathrm{C}_{\mathrm{f}}$ for circular tank with horizontal baffles in a TOP lane change maneuver ............72

Figure 68. Peak moments for circular tank with vertical baffles in a TOP lane change

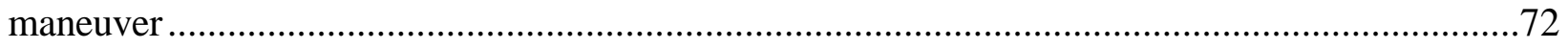

Figure $69 . \mathrm{C}_{\mathrm{m}}$ for circular tank with vertical baffles in a TOP lane change maneuver ..............73

Figure $70 . \mathrm{C}_{\mathrm{f}}$ for circular tank with vertical baffles in a TOP Lane change maneuver...............73

Figure 71. Peak moments for circular tank with horizontal-vertical baffles in a TOP lane

change maneuver

Figure 72. $\mathrm{C}_{\mathrm{m}}$ for circular tank with horizontal-vertical baffles in a TOP lane change

maneuver 
Figure $73 . \mathrm{C}_{\mathrm{f}}$ for circular tank with horizontal-vertical baffles in a TOP lane change

maneuver .75

Figure 74. Peak moments for circular tank with diagonal baffles in a TOP lane change

maneuver .76

Figure $75 . C_{m}$ for circular tank with diagonal baffles in a TOP lane change maneuver. .77

Figure $76 . \mathrm{C}_{\mathrm{f}}$ for circular tank with diagonal baffles in a TOP lane change maneuver. .77

Figure 77. Peak moments for elliptical tank with horizontal baffles in a TOP lane change maneuver .79

Figure $78 . \mathrm{C}_{\mathrm{m}}$ for elliptical tank with horizontal baffles in a TOP lane change maneuver 80

Figure $79 . \mathrm{C}_{\mathrm{f}}$ for elliptical tank with horizontal baffles in a TOP lane change maneuver .80 Figure 80. Peak moments for elliptical tank with vertical baffles in a TOP lane change maneuver .81

Figure $81 . \mathrm{C}_{\mathrm{m}}$ for elliptical tank with vertical baffles in a TOP lane change maneuver........ .81

Figure $82 . \mathrm{C}_{\mathrm{f}}$ for elliptical tank with vertical baffles in a TOP lane change maneuver .82

Figure 83. Peak moments for elliptical tank with horizontal-vertical baffles in a TOP lane change maneuver. .82

Figure $84 . \mathrm{C}_{\mathrm{m}}$ for elliptical tank with horizontal-vertical baffles in a TOP lane change

maneuver .83

Figure $85 . \mathrm{C}_{\mathrm{f}}$ for elliptical tank with horizontal-vertical baffles in a TOP lane change maneuver .83 
Figure 86. Peak moments for elliptical tank with diagonal baffles in a TOP lane change maneuver

Figure $87 . \mathrm{C}_{\mathrm{m}}$ for elliptical tank with diagonal baffles in a TOP lane change maneuver .85

Figure $88 . \mathrm{C}_{\mathrm{f}}$ for elliptical tank with diagonal baffles in a TOP lane change maneuver .85

Figure 89. Percentage ratios of peak moments in an elliptical tank with diagonal baffles

to peak moments without any baffles .93

Figure 90. Moment with time for various baffles $(1 / \mathrm{a}=0.75)$ in an elliptical tank with $30 \%$

fill level

Figure 91. Percentage ratios of peak moments in a circular tank with horizontal baffles to peak moments without any baffles in TOP lane

Figure 92. Percentage ratios of peak moments in a circular tank with vertical baffles to peak moments without any baffles in TOP lane

Figure 93. Percentage ratios of peak moments in a circular tank with horizontal-vertical baffles to peak moments without any baffles in TOP lane .95 Figure 94. Percentage ratios of peak moments in a circular tank with diagonal baffles to peak moments without any baffles in TOP lane 


\section{NOMENCLATURE}
a $=$ Smaller radius of the tank
a = Lateral acceleration
$\mathrm{b} \quad=$ Larger radius of the tank
$\mathrm{C}_{\mathrm{m}} \quad=$ Dynamic coefficient of Moment
$\mathrm{C}_{\mathrm{f}} \quad=$ Dynamic coefficient of Force
D $\quad=$ Diameter of the tank
g = Acceleration due to gravity
1 = Length of the baffle
1/a = Ratio of length of the baffle to smaller radius of the tank
$\mathrm{M}=$ Moment
$\mathrm{R}=$ Radius of the tank
$\delta, \xi=$ Damping factor
$\mathrm{v} \quad=$ Kinematic viscosity
$\mathrm{V} \quad=$ Velocity of the tank 


\section{CHAPTER 1 \\ INTRODUCTION AND PROBLEM STATEMENT}

\subsection{Introduction}

Heavy-duty tanker trucks carrying liquid cargo have poor overturning and skidding stability because of the high center of gravity and sloshing of the liquid. The problem of instability is further exacerbated when the trailer is subjected to various dynamic maneuvers, such as lane change, uturns and braking in a turn or cornering. Roll over instability is particularly sensitive to the dynamic sloshing effects in partially filled tanks. Lack of lateral force feedback from the trailer further reduces the overall safety. Thus, these two conditions namely, high center of gravity and the dynamic sloshing of the liquid cargo have placed heavy-duty tanker trucks in a very high-risk category on the roadways.

The geometric design of tank containers (elliptical and cylindrical), the presence of baffles in various configurations, different types of suspensions and tank fill levels have a great influence on the rollover stability of tanker trucks for maneuvers that are typically safe for trucks with fixed load. The consequences of heavy vehicle overturning and skidding accidents are especially critical when they are associated with severe human injuries and considerable economic losses. If the accident causes a toxic load to leak from its container, the environment as a whole may be damaged and threatened. Therefore, the maneuver performance and active safety of heavy vehicles should be as good as for other 
vehicles. But unfortunately this is generally not the case because of certain design characteristics mentioned above.

In conventional tanks where, the length is much greater than the width, the internal liquid motions can be more severe longitudinally than laterally if no transverse walls are present partitioning the tank. The longitudinal acceleration peaks (maximums) might be larger than the lateral peaks because of more length than width. Hence legislation has till now concentrated only on reducing the longitudinal slosh requiring transverse walls in tankers. However, the stability can be more sensitive to lateral sloshing. Wheel load transfer and overturning tendency can be more dominating laterally than longitudinally.

Longitudinal sloshing in it self is more abrupt and easily perceivable to the driver even if it occurs in the trailer. Lateral sloshing is less abrupt and more difficult to perceive when it occurs in the trailer. Hence it is unlikely that the driver can adapt the steering in complex maneuvers to avoid forces generated because of lateral sloshing.

\subsection{Objectives}

This project aims at studying the effect of baffles in reducing the lateral sloshing and thereby improving the stability of heavy-duty tanker trucks. Various configurations (horizontal, vertical, horizontal and vertical and diagonal) of baffles were considered for this purpose. The objective of this project was to determine the best configuration of baffles for various shapes of tank-containers, percent fill level and different kinds of maneuvers. 
Analysis of dynamic sloshing involves identification of flow patterns inside the tankers, followed by finding a solution to complex non-linear governing equations using the appropriate boundary conditions. Computational Fluid Dynamics tools FLUENT and GAMBIT are used for the purpose. Two shapes of tank, circular and elliptical, are modeled with four different fill levels of 30, 50, 70 and 90 percent of the vertical dimension of the tank. These models are solved both in a constant radius turn and a TOP lane change maneuver with four kinds of baffles mentioned earlier.

Once the best baffle configuration was obtained for an elliptical tank, it was proposed hat a full-scale army tanker with these baffles be built and find the effect in a constant radius turn and TOP lane change maneuver.

Another objective of this study was to determine the damping effect of dynamic viscosity of the liquid, inside the tank, on lateral sloshing. This would be helpful in determining the trammel pendulum parameters for pendulum-fluid dynamic effect matching. Trammel pendulums can be used to effectively predict the rollover threshold of partially filled heavy-duty tanker trucks. A torsional damper can be incorporated in the trammel pendulum based on the effect of dynamic viscosity of the liquid on damping the liquid sloshing.

\subsection{Scope}

Computational fluid models were developed using FLUENT and GAMBIT and these models were solved on a personal computer with $256 \mathrm{MB}$ RAM and Pentium 
Processor with $650 \mathrm{MHz}$ speed. Dynamic coefficients of moment and force and Frequency of liquid oscillation were calculated for an elliptical tank undergoing a steady turning with a lateral acceleration of $0.3 \mathrm{~g}$, which is the critical acceleration moving the vehicle towards unstability. These values were verified with those obtained from the numerical solution of the 2-D Navier-stokes, continuity and 1-D free-surface equations [16]. The values were also compared with the values obtained from DYNA models. Once the models were verified they were used extensively to determine the effect of baffles.

Finding the peak values of moment and lateral force was the most important thing in this research. Comparing the maximum values of moment and force in the models with various baffles with the same in the models without baffles gives an idea of the effect of baffles on reducing sloshing. Two maneuvers namely, constant radius turn and TOP Lane change maneuver are considered for this purpose.

This study does not delve into structural aspects of the baffles inside the tank. It is confined to a determination of the best baffle configuration for a particular fill level of a circular/elliptical tank maneuvering a constant radius turn, and a TOP lane change maneuver. 


\section{CHAPTER 2}

\section{LITERATURE REVIEW}

It is common knowledge that any small container filled with liquid must be moved or carried very carefully to avoid spills. The unrestrained free surface of the liquid has an alarming propensity to undergo large excursions for even small motions of the

container. Similar phenomenon surrounds us at almost every turn. Almost any moving vehicle provides an example wherein the response of a contained liquid may be of concern: I) aircraft and rocket fuel tanks; II) cargo, ballast, or fuel tanks of large ships; III) fuel or cargo tanks of automotive vehicles; IV) railroad tank cars, V) heavy duty tanker trucks, etc.

In heavy-duty tanker trucks the response of the vehicle to motions of the contained liquid may reduce the stability of the vehicle greatly. The oscillations, when allowed to continue, can create slosh forces that may have an adverse effect on the stability and structural integrity of the vehicle. In particular, if the liquid masses are excited at a frequency near that of a lower liquid mode, the amplitude of the liquid oscillations, and hence the resultant forces and moments, may be of such magnitude so as to induce unstability to the vehicle. When liquid sloshing occurs in a spherical tank, for example, the maximum slosh forces approach a value equal to approximately one-fourth of the apparent weight of the contained liquid [1]. 
Positive expulsion bags, diaphragms, floating cans and asymmetrical or annular ring baffles are among several methods that have been investigated in efforts to increase the damping of a liquid in a container. In fact, fuel tanks of all the racing cars in the Indianapolis 500 of 1965 contained hollow plastic ball-like devices ("wiffle" balls) intended to prevent fuel sloshing [2].

Another method of increasing the damping applied to the contained liquid is the use of the viscous damping inherent in high viscous liquids. An experimental investigation was conducted at the NASA Lewis Research Center to study the potential slosh-damping effectiveness of viscous liquids having a range of kinematic viscosities from $1.23 \times 10^{-6}$ to $1.183 \times 10^{-2}$ square foot per second [1]. Liquids used for this purpose were water, glycerin and a mixture of these two liquids in varying percentages. The above investigation was conducted utilizing three rigid spherical tanks of 9.5, 20.5 and 32 inches in diameter. Effects of liquid kinematic viscosity, as well as excitation amplitude and frequency on damping ratios were investigated. The following conclusions were drawn from the experiments conducted.

1. The damping ratios obtained for each tank diameter increased with the kinematic viscosity of the contained liquid and were essentially independent of the excitation amplitudes investigated.

2. For a constant value of the kinematic viscosity, the damping ratios decreased as the tank diameter increased. 
The damping ratios obtained in this study [1] over a range of liquid kinematic viscosities from $0.1062 \times 10^{-4}$ to $118.3 \times 10^{-4}$ were in between 0.05 to 0.25 . The damping ratio increased with kinematic viscosity. A general expression was found for the first mode damping ratio in terms of kinematic viscosity and the tank diameter. The expression was given as:

$$
\delta=0.131\left(\frac{v \times 10^{4}}{\sqrt{g D^{3}}}\right)^{0.359}
$$

In another experimental study [3] conducted at the Langley Research Center, Va by NASA it was found that kinematic viscosity of the liquid had no apparent effect on damping for the range of kinematic viscosity considered. Figure 1 illustrates the findings of this study [3].

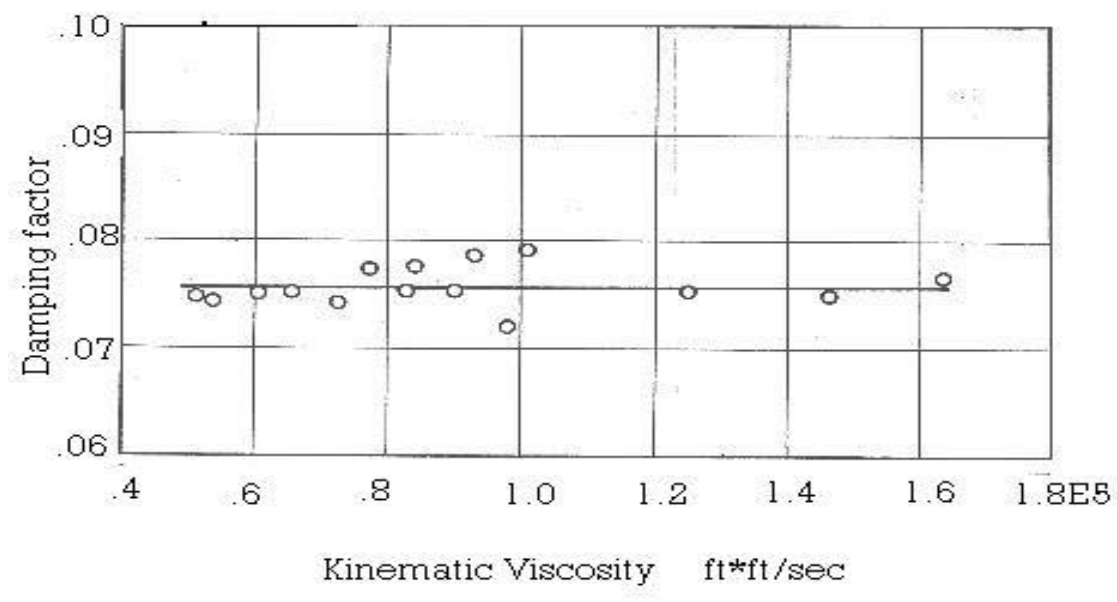

Figure 1. Variation of damping factor with kinematic viscosity of water for a fixed ring baffle mounted in 30-inch diameter tank [3]. 
All the tanks used in the NASA investigation [3] were all spherical in shape, and they were subjected to a sinusoidal motion with the help of a slider-crank mechanism driven through a variable speed transmission by an electric motor. These results can not be used to predict the viscous damping in a heavy duty tanker truck as the trailer carrying the liquid goes through a different maneuver altogether. The excitation is also totally different in this case. The liquid is subjected to a constant lateral acceleration in both constant radius turn and double lane change maneuvers. Hence, this study was aimed at investigating the effect of viscous damping on fluid sloshing in tanks of various shapes maneuvering a constant radius turn, and double lane change maneuver. Details of this study are presented in the first part of this report.

Viscous damping is inherently present in a liquid. This takes place due to the energy dissipation that always occurs during the liquid oscillations. But, the amount of damping offered by this energy dissipation is not large enough. The damping effectiveness of movable and floating devices has been studied by several investigators [4-10]. Although such devices can substantially damp liquid oscillations they also involve significant weight penalties. Positive expulsion bags and diaphragms of elastomeric material were investigated by Stofan $[4,5]$. It was found that significant damping could be obtained but was strongly dependent on diaphragm thickness. Expulsion bags performed similar to the diaphragms.

A significant amount of effort $[3,2]$ has been devoted to investigation of damping of liquid oscillations by various types of fixed baffles. Baffles provide large damping for relatively low weight without other problems. Published literature [19] presents related 
information pertaining to space-flight boosters in which liquid-fueled propulsion systems are used.

The effects of baffle configuration, width, thickness, flexibility and location on the damping and frequency of the fundamental anti-symmetric mode of liquid oscillations are important. Analytical means of predicting damping are not only tedious but also difficult because of the complexity in boundary conditions and the presence of baffles makes it even tougher.

An experimental investigation of the damping characteristics was conducted by Silveira et al. [3] at the NASA Langley Research Center. The types and dimensions of the six different baffles considered by Silveira et al. [3] in a cylindrical tank are shown in Figure 2. The liquid was excited by means of a paddle. When a sufficient amount of liquid amplitude is reached the excitation was stopped. The rate of decay of moments resulting form liquid oscillatıons was then measured using strain gages and load cells.

Experiment results [3] showed that the location of the flat ring and conic section baffles for effective damping was restricted to a narrow region near the surface of the liquid. At this location the damping increased with size of flat ring and conic section baffles. The cruciform baffles offered low damping compared to other baffles of comparable size. The effect orientation was found to be small. Fixed ring baffles appeared to offer highest mean damping factor based on total surface area of the baffles. All the results [3] are summarized in Figure 3. 

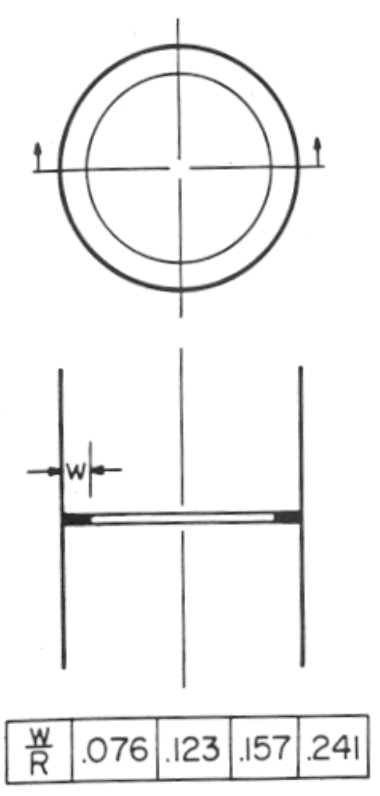
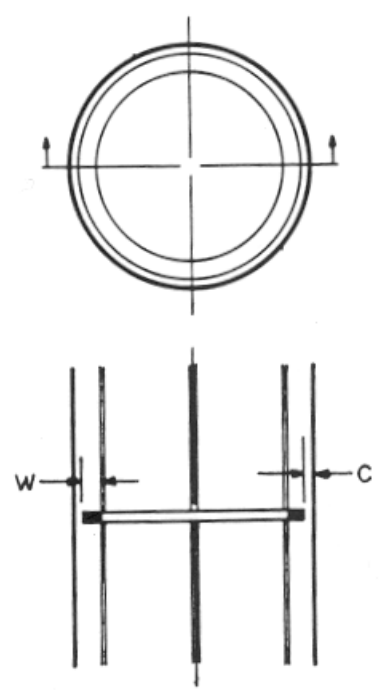

\begin{tabular}{|l|l|l|l|l|l|}
\hline$\frac{W}{R}$ & .084 & .084 & .084 & .126 & .169 \\
\hline$\frac{C}{R}$ & .021 & .042 & .084 & .021 & .021 \\
\hline
\end{tabular}
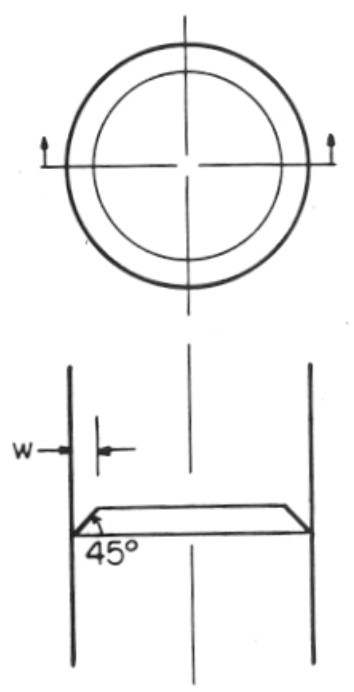

\begin{tabular}{|l|l|l|l|l|}
\hline$\frac{W}{R}$ & .076 & .119 & .157 & 241 \\
\hline
\end{tabular}

(a) Fixed ring. (b) Ring with radial clearance.

(c) Conic section.
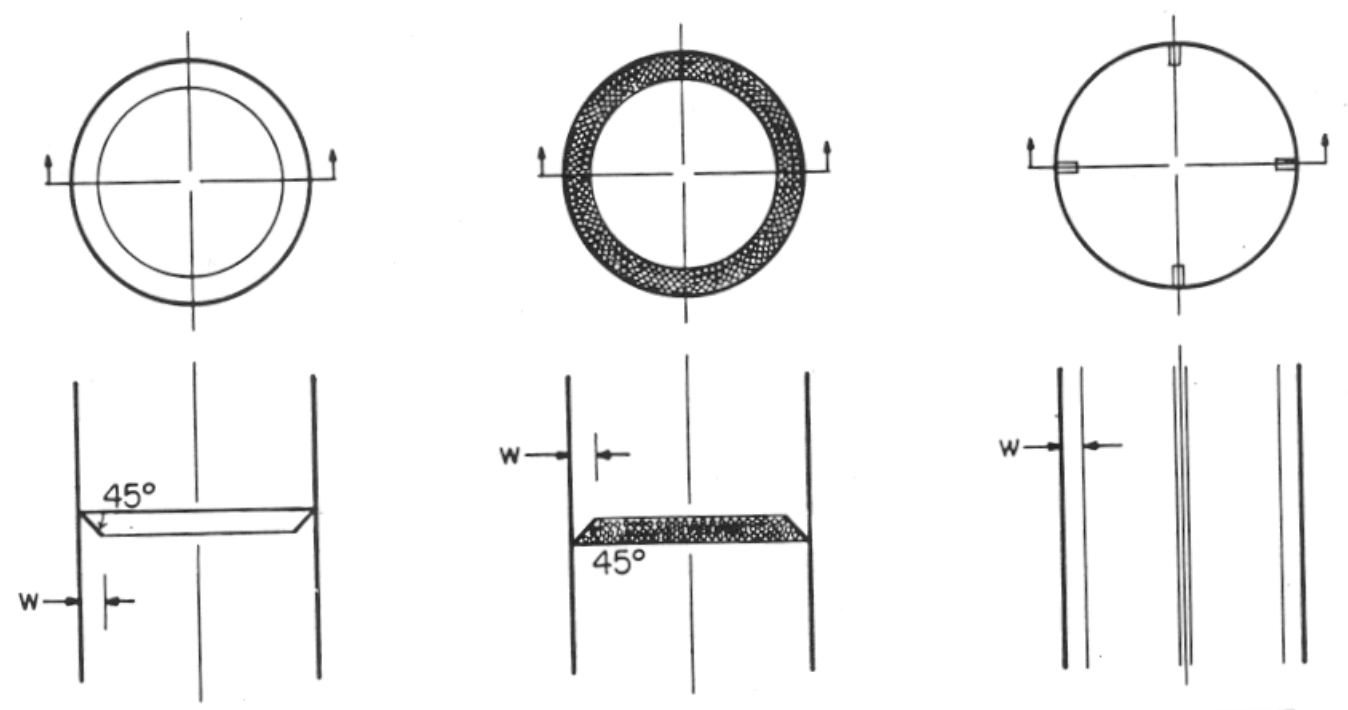

$$
\begin{array}{|l|l|}
\hline \frac{W}{R} & .157 \\
\hline
\end{array}
$$

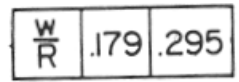

\begin{tabular}{|l|l|l|}
\hline $\mathrm{W}$ & .169 & .337 \\
\hline
\end{tabular}

(e) Perforated conic section.

Figure 2. Baffle configurations [3] 


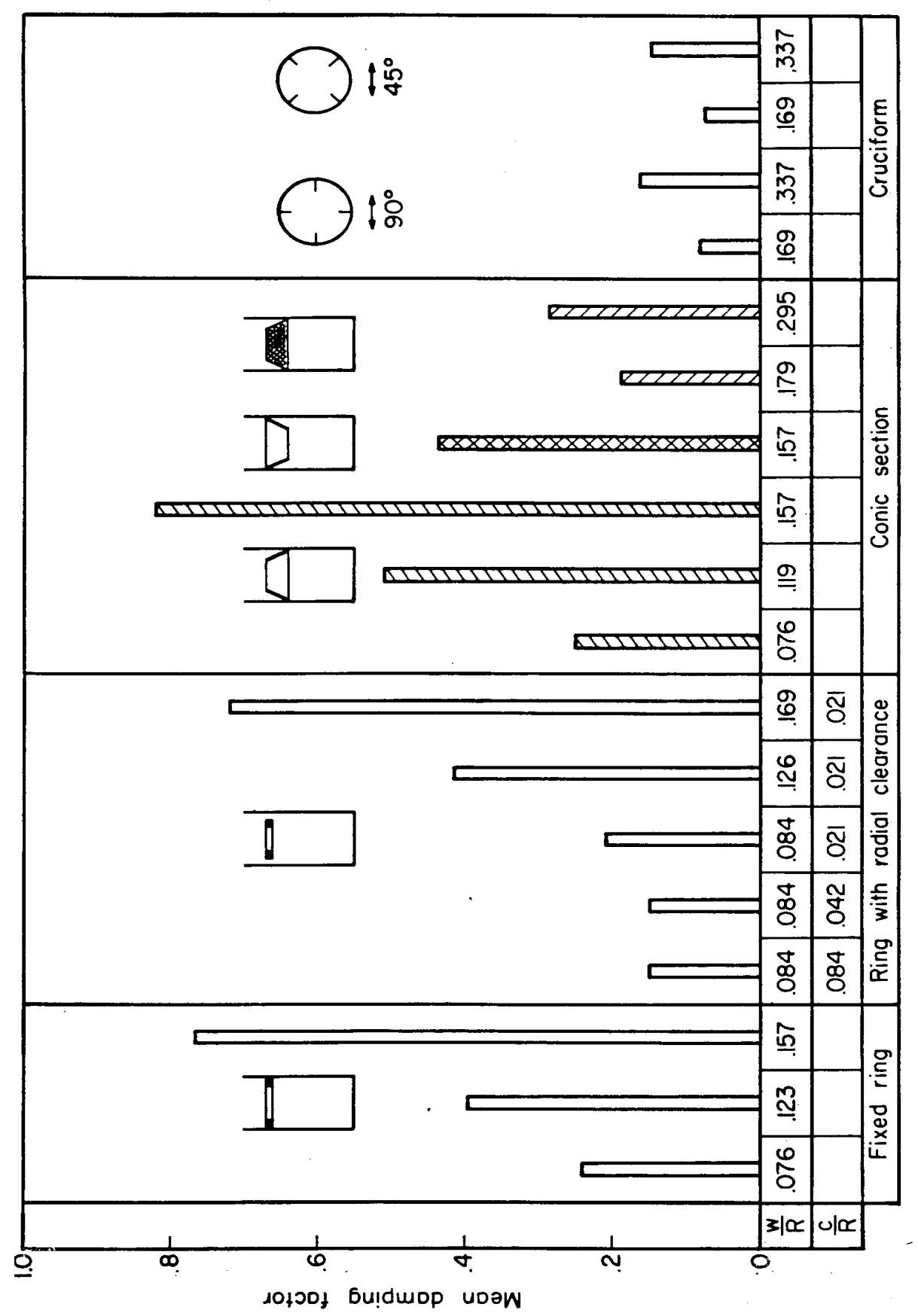

Figure 3. Comparison of baffles [3]

All the investigations reported above were targeted towards space vehicles and the excitation was sinusoidal. There is very sparse information in published literature on baffles in heavy-duty tanker trucks. Bauer [11] illustrated the effects of compartmentation for a rectangular cylinder. Bauer [11] showed that a sub-division of a container by cross 
walls increased the fundamental frequency and reduced the sloshing mass. Hence, enhancing the vehicle stability. Using small-scale models, and a mathematical model, Strandberg [12] studied the influence of large amplitude sloshing on overturning and skidding stability of road tankers. Lidstrom [13] also studied the same topic. For steady state cornering they found that a partly loaded circular tank is more stable than a rectangular tank. Their results are shown in the following Figure 4 Shaded, partly hidden bars represent a rigid load fixed to the tank, and white bars represent unrestrained liquid. Black narrow bars represent $0.5 \mathrm{~Hz}$ harmonic oscillations for circular tank in (a) and for elliptic tank (not rectangular) in (b) and (c). The stabilization effect from cross walls, in (c), was found for harmonic oscillation as well and is also shown in Figure 4 [13].

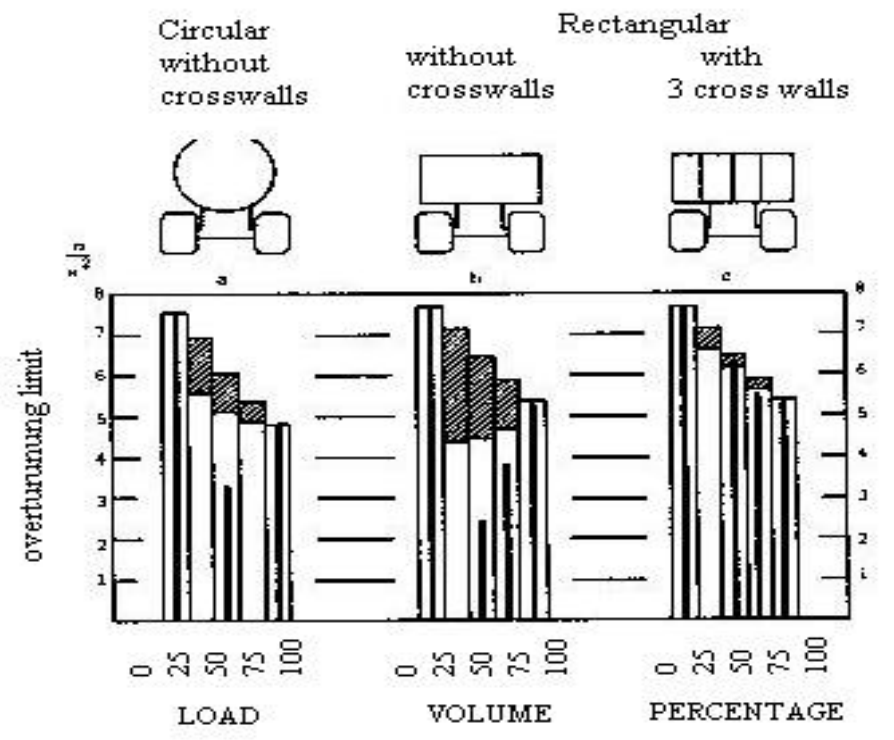

Figure 4. Overturning limits for steady state cornering [13]

Popov et al. [14] discussed the effect of compartments and baffles in rectangular containers for uniform breaking and steady cornering maneuvers. The influence of number of separating walls, size and location of orifices in the separating walls was 
studied. Steady state response of the liquid was obtained analytically whereas the transient solution was obtained by a numerical solution of the incompressible 2-D Navier-Stokes, continuity and the free surface differential equations. Popov et al. [14] found that the influence of compartments in reducing the sloshing amplitudes is significant, while baffles had a somewhat lower effect because of the fluid coupling between compartments. The location of orifice in case of a baffle is found to be unimportant if it was totally immersed in the liquid. If the orifice is partially filled then it also played a significant role in reducing the sloshing similar to compartments. Some of their results are shown in the Figure 5. This would reduce the longitudinal sloshing but not the lateral sloshing.

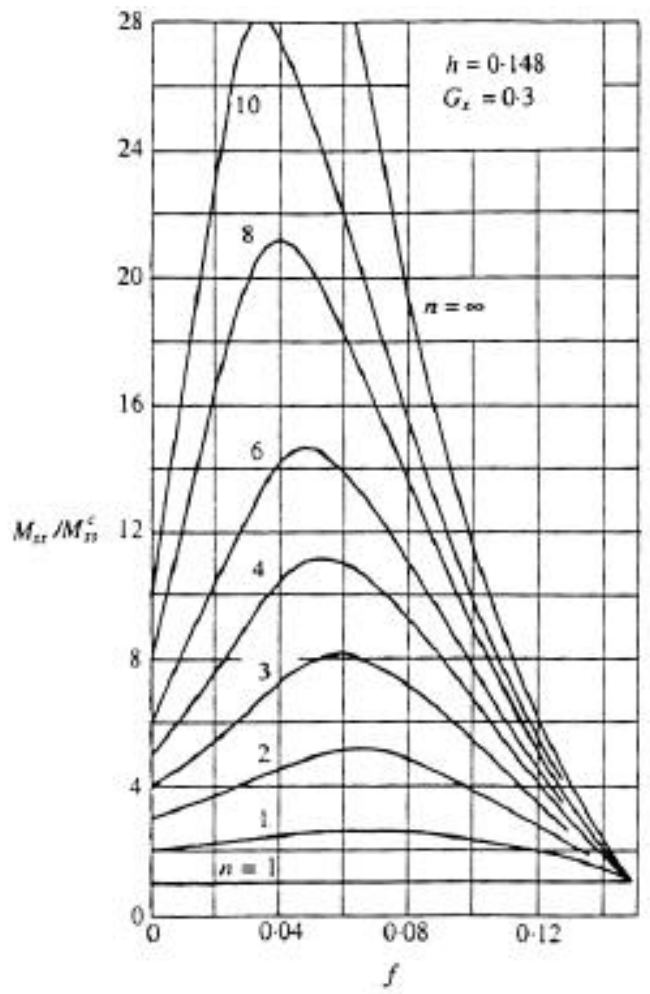

Figure 5. Ratio of steady state overturning moment of an uncompartmented to compartmented tank [14] 
Ibrahim [15] studied the effect of partitions containing rectangular slots and holes of different sizes, anti-slosh damper, to generate additional damping. Figure 6 shows one such anti-slosh damper. He constructed a small-scale experimental model for a cylindrical truck tank, and excited the contained fluid in the lateral direction, to measure the viscous damping and the damped natural frequency of the vehicle. A mathematical model was then developed for a rigid truck having 3 axles and carrying a cylindrical liquid container based on similarity technique for predicting the dynamic behavior of the cylindrical container. Ibrahim [15] concluded that a partition with vertical slots may be used as an energy dissipative unit. Such a slotted partition fit through the longitudinal plane increases the potential to damp the liquid oscillations in the lateral directions with the decrease of slots size. It was also found that the rollover threshold acceleration limit could be increased. Some of his results [15] are shown in Figures 7, 8, and 9.

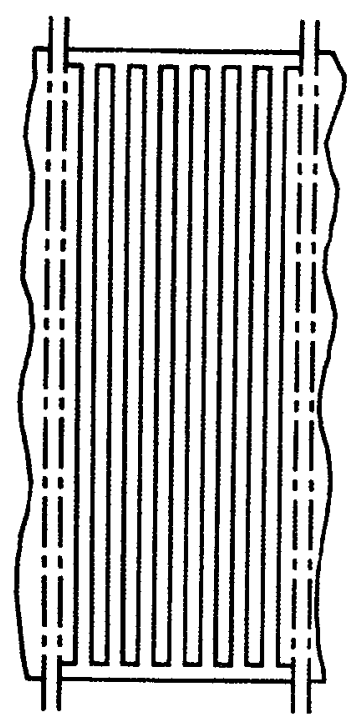

Figure 6. Slotted partition [15] 


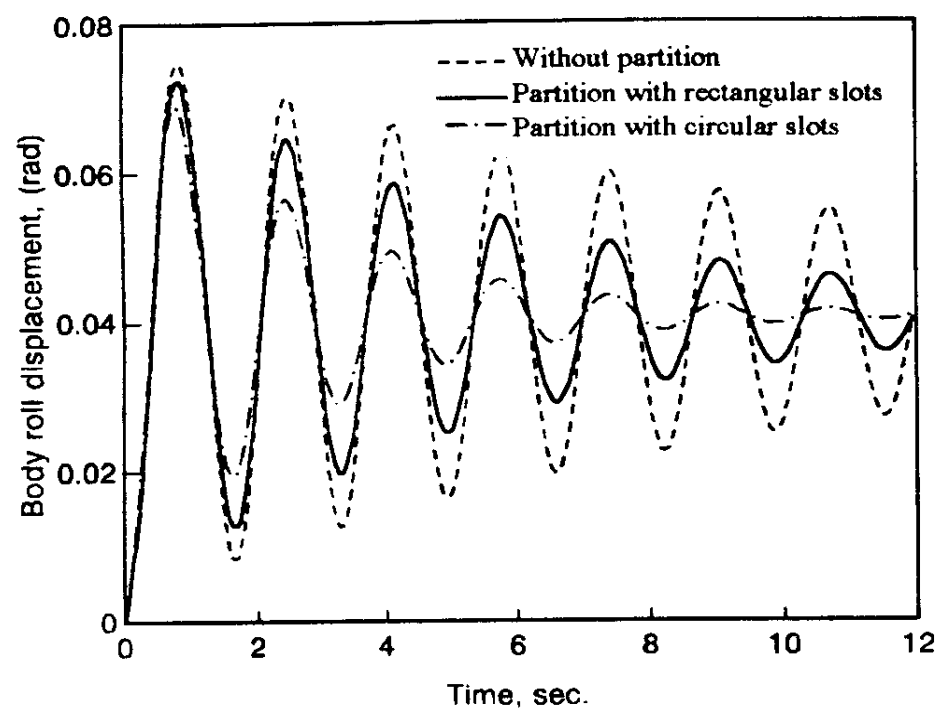

Figure 7. Body Roll Displacement [15]

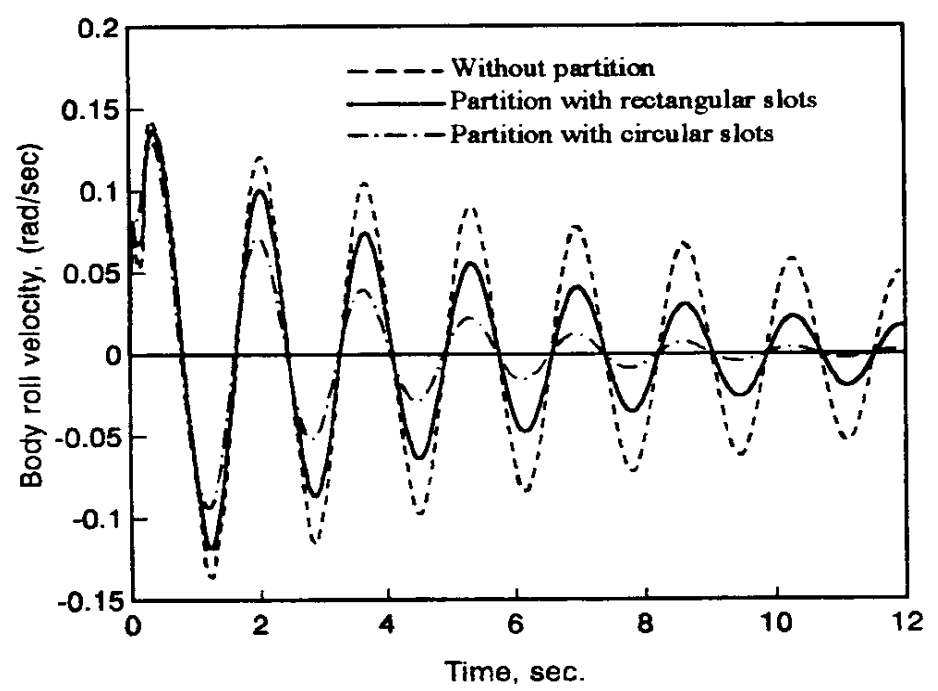

Figure 8. Body roll Velocity [15] 


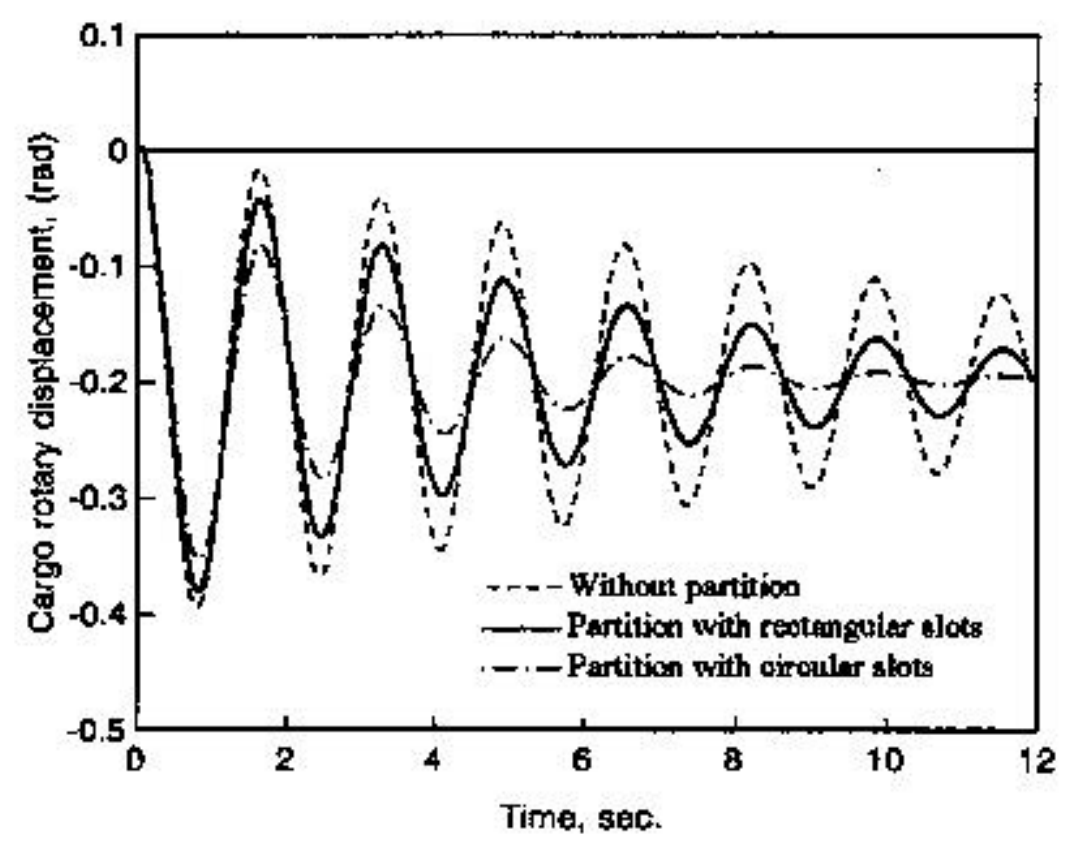

Figure 9. Cargo rotary displacement [15]

A review of published literature shows that very limited amount of work has been reported on reduction of lateral sloshing of liquids in heavy-duty road tanker trucks. This study explores the effect of fixed baffles in reducing the lateral sloshing of liquid in heavy-duty tanker trucks. To the best of this author's knowledge, this is a first attempt at modeling the dynamic sloshing using computational fluid dynamics techniques (FLUENT) in an attempt to optimize baffles. 


\section{CHAPTER 3}

\section{MODEL DESCRIPTION \& VERIFICATION}

\subsection{Model Description}

Fluid models of the elliptical tank with various aspect ratios were modeled using GAMBIT 1.2 and FLUENT 5.4. The tank geometry with various baffles and different aspect ratios was modeled using GAMBIT. These models were then meshed using hexahedral elements and exported to FLUENT for further development.

FLUENT is a general purpose computer program for modeling fluid flow, heat transfer and chemical reaction. It should be noted that structural analysis cannot be performed using FLUENT. The purpose of developing these models was to generate the forces and moments, acting on the tank walls, as a result of liquid sloshing inside. These values of forces and moments could then be used in finite element models to determine the threshold velocity and accelerations for a full tanker-truck combination. Hence the entire truck with tractor and trailer need not be modeled. Modeling just the tanks would be enough for analyses carried out in this project. Both two-dimensional and threedimensional elliptical tanks with different aspect ratios and with/without baffles were modeled using GAMBIT. Geometry of the tank is more important here because the fluid flow within the tank is affected mainly by the geometry. These tank models were then meshed using hexahedral elements. Boundary zones (walls) were then defined and exported to FLUENT. Once the models were exported to FLUENT, the case file was 
built using Volume of Fluid (VOF) Free Surface Model. This VOF model is used when two or more types of fluids are present. VOF model relies on the fact that the fluids do not interpenetrate. The two fluids used in the present study were air and water. The fluids share a single set of momentum equations and the volume fraction of each computational cell is tracked throughout the domain.

\subsection{Building the case file}

\subsubsection{Solver}

A segregated solver was used for the solution algorithm of these unsteady problems as the coupled solver cannot be used with multiphase models. The volume of fluid model, used in this study is a multi phase model. In a segregated solver the governing equations are solved sequentially (i.e. segregated form one another) whereas in a coupled solver they are solved simultaneously. Implicit formulation had to be used, as the explicit formulation is available only for the coupled solver. Absolute velocity formulation was used in this case. The relative velocity formulation is appropriate when most of the fluid in the domain is rotating, as in the case of a large impeller in a mixing tank. A transient analysis was done with the above parameters set. Figure 10 shows the various parameters set in the solver panel of the FLUENT. 

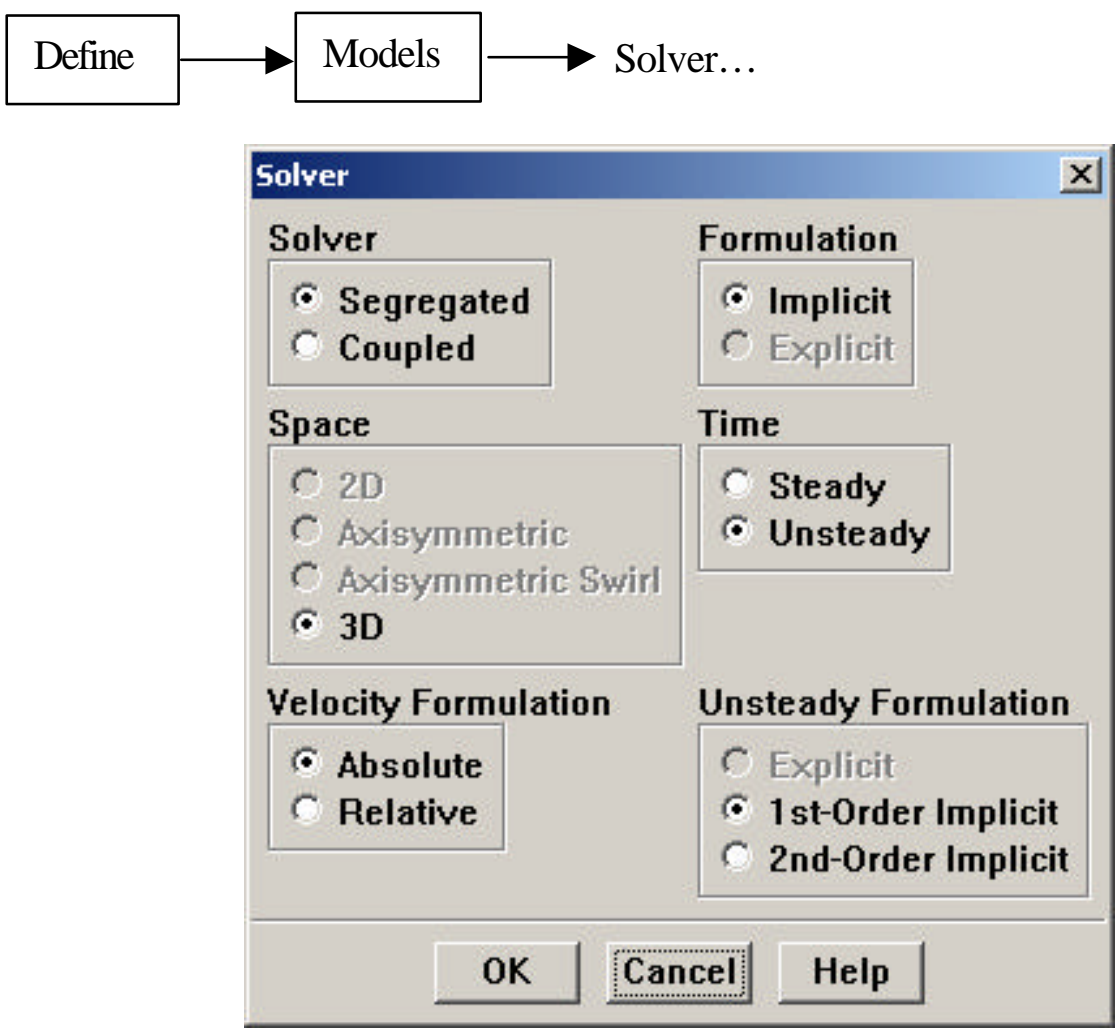

Figure 10. Solver panel in FLUENT

\subsubsection{Physical Model}

The next step in the model development was specifying the turbulence model. The standard $\kappa-\varepsilon$ model was used in this problem. Robustness, economy and good accuracy for a wide range of flow problems were the factors, which influenced the selection of the $\kappa-\varepsilon$ model. Water was then imported form the materials database to be available to be used for the secondary phase. Air was used as the primary phase. 


\subsubsection{Volume of Fluid Model}

VOF free surface model was activated in the multiphase model panel after specifying the turbulence model. A reasonable courant number of 0.5 was used in the analyses. The Courant number is a dimensionless number that compares the time step in a calculation to the characteristic time of transit of a fluid element across a control volume given by the equation:

$$
\frac{\Delta t}{\Delta x_{\text {cell }} / N_{\text {fluid }}}
$$

The total number of phases in the model was specified as 2 with air as the primary phase and water in the secondary phase. Water was defined as the secondary phase mainly for the convenience in setting up the problem. When we initially patch the volume fraction in the bottom half of the tank, where liquid is, it is more convenient to patch a water volume fraction of unity there than to patch volume fraction of air as unity in the rest of the tank.

The next step is to specify the various VOF parameters in multiphase model panel. These parameters influence the prediction of interface between water and air. There are 4 different VOF formulations available in FLUENT for time dependent solutions: i) Implicit ii) Explicit iii) Donor-Acceptor and iv) Geometric Reconstruction. The third scheme, donor-acceptor is used if the mesh contains highly twisted hexahedral cells. The models in this problem do not have any such cells. Hence, it was not a suitable scheme for this problem. Implicit interpolation scheme can be used if the intermediate transient solution is of no interest and only final steady state solution is of importance. 
The most accurate interface-tracking scheme is the Geometric-Reconstruction scheme and is recommended for most VOF calculations. Euler explicit formulation is computationally less expensive than Geometric-Reconstruction but the interface between phases is not as sharp as that predicted with Geometric-Reconstruction scheme. Hence, Geometric-Reconstruction scheme was used in all the models that were developed in this study. Figure 11 shows the multiphase model panel in FLUENT with all the associated parameters set.

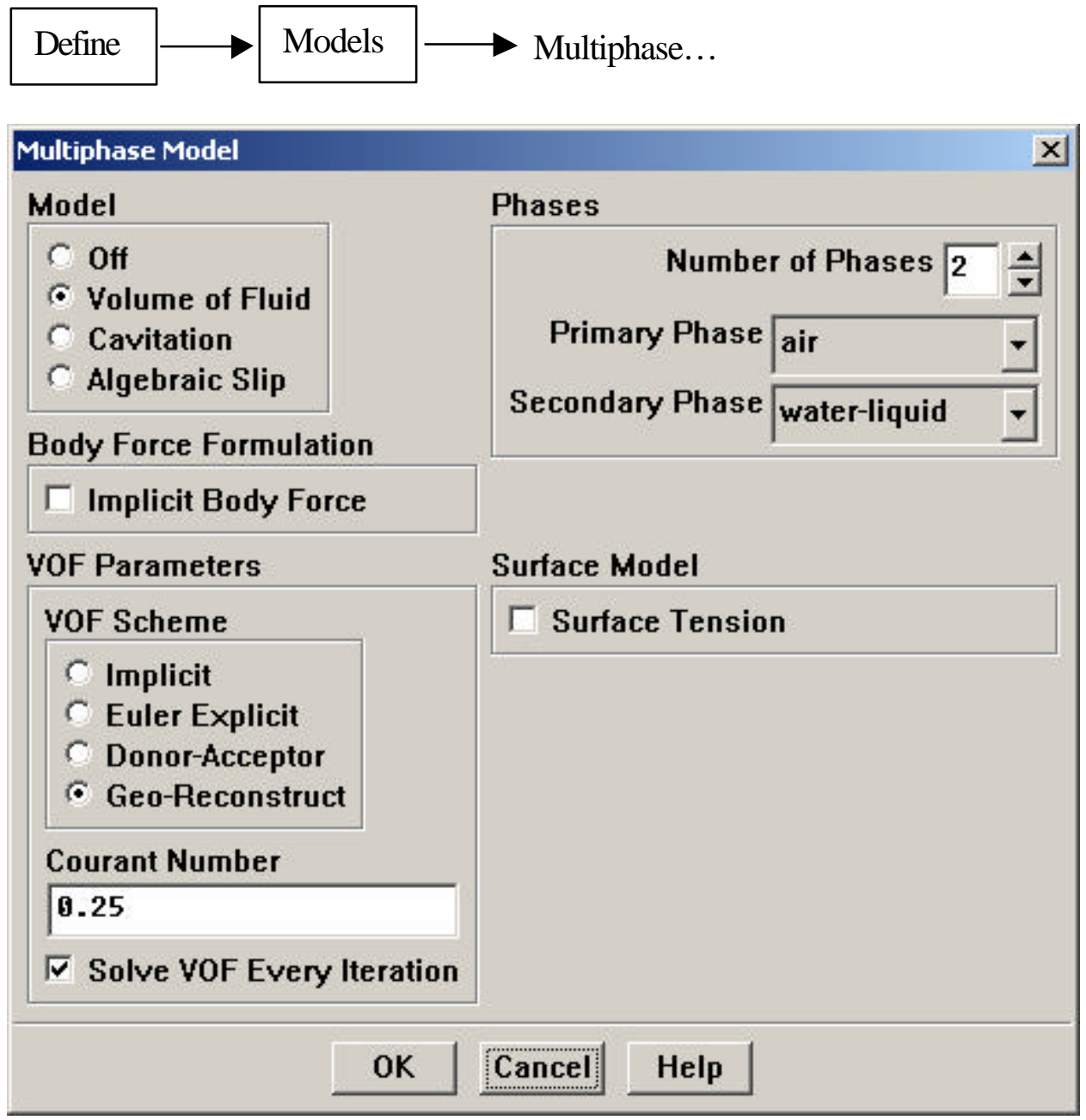

Figure 11. Multiphase model panel in FLUENT 


\subsubsection{Body Forces}

Within a tanker, fluid motion occurs due to both gravitational and external forces. To include body forces Fluent has the option of including 'Geometry' in the operating conditions panel and allows gravitational acceleration to be specified. The operating density option (available within the software package) was used and the operating density was set to 1.225 , which is the density of the lighter phase, air. This excludes the buildup of hydrostatic pressure within the lighter phase, improving the accuracy for momentum balance. The appropriate boundary conditions were then applied and initial volume fraction of water was patched after initializing the problem. Figures 12 shows the operating conditions panel of FLUENT with appropriate values of associated parameters.

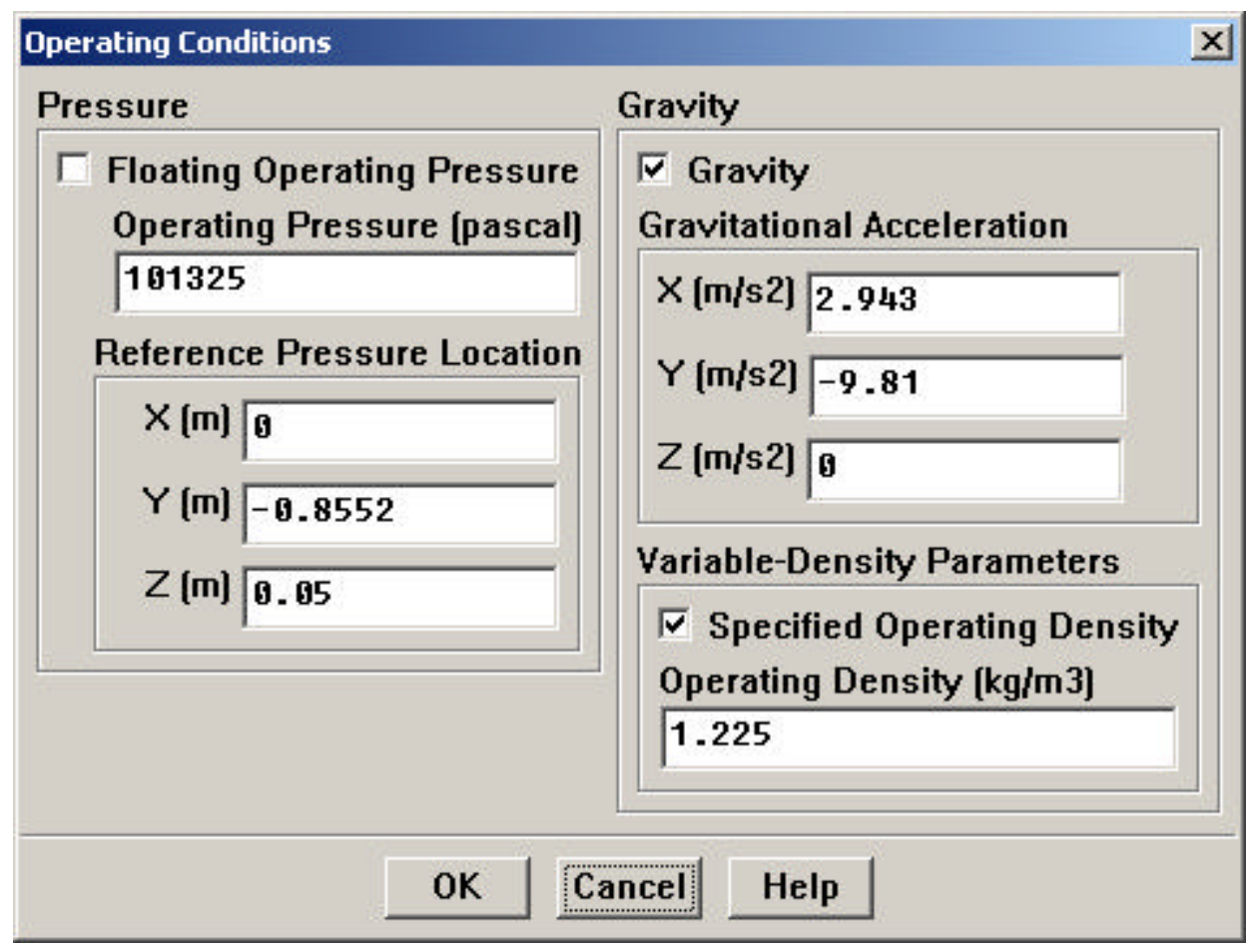

Figure 12. Operating conditions panel in FLUENT 
Figure 13 shows the initial volume fraction of water in a circular tank with $30 \%$ fill level. The bottom portion of the tank is patched with water. A solution is carried out for this model with other solution control parameters set in the solution controls panel explained in the next section.

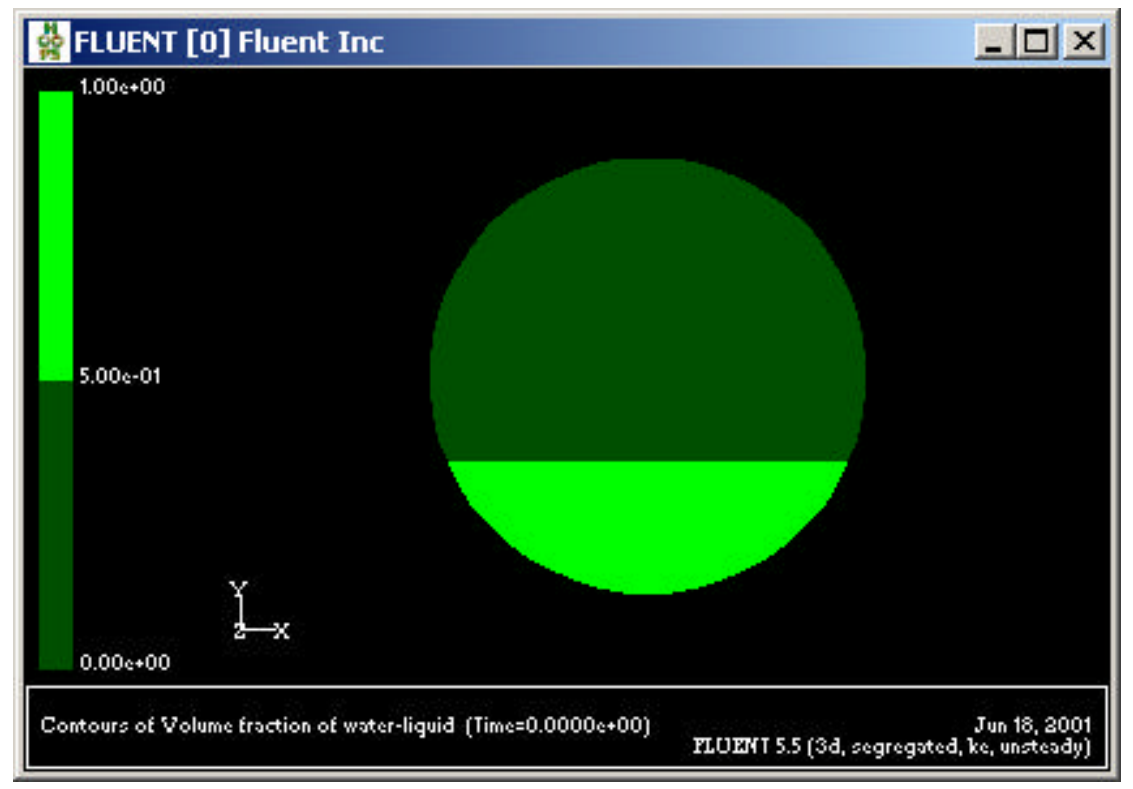

Figure 13. Volume fraction of water in a circular tank with $30 \%$ fill level

\subsubsection{Solution Strategies}

Efforts were made to improve the accuracy and convergence of the VOF solution. The reference pressure location was set at the bottom center of the tank where only water is present at all times. This is a condition essential for smooth and rapid convergence. The PISO (Pressure-Implicit with Splitting of Operators) was used for pressure-velocity coupling. This allowed for increased values on all under-relaxation factors without loss of 
solution stability. All the under-relaxation factors were set to unity for faster convergence of the solution. The values were reduced later if the solution was not converging. Figure 14 shows the various solution parameters set in the solution controls panel. The models were then solved for both a constant radius turn and a TOP lane change maneuver for appropriate time steps with 20 iterations in each time step.

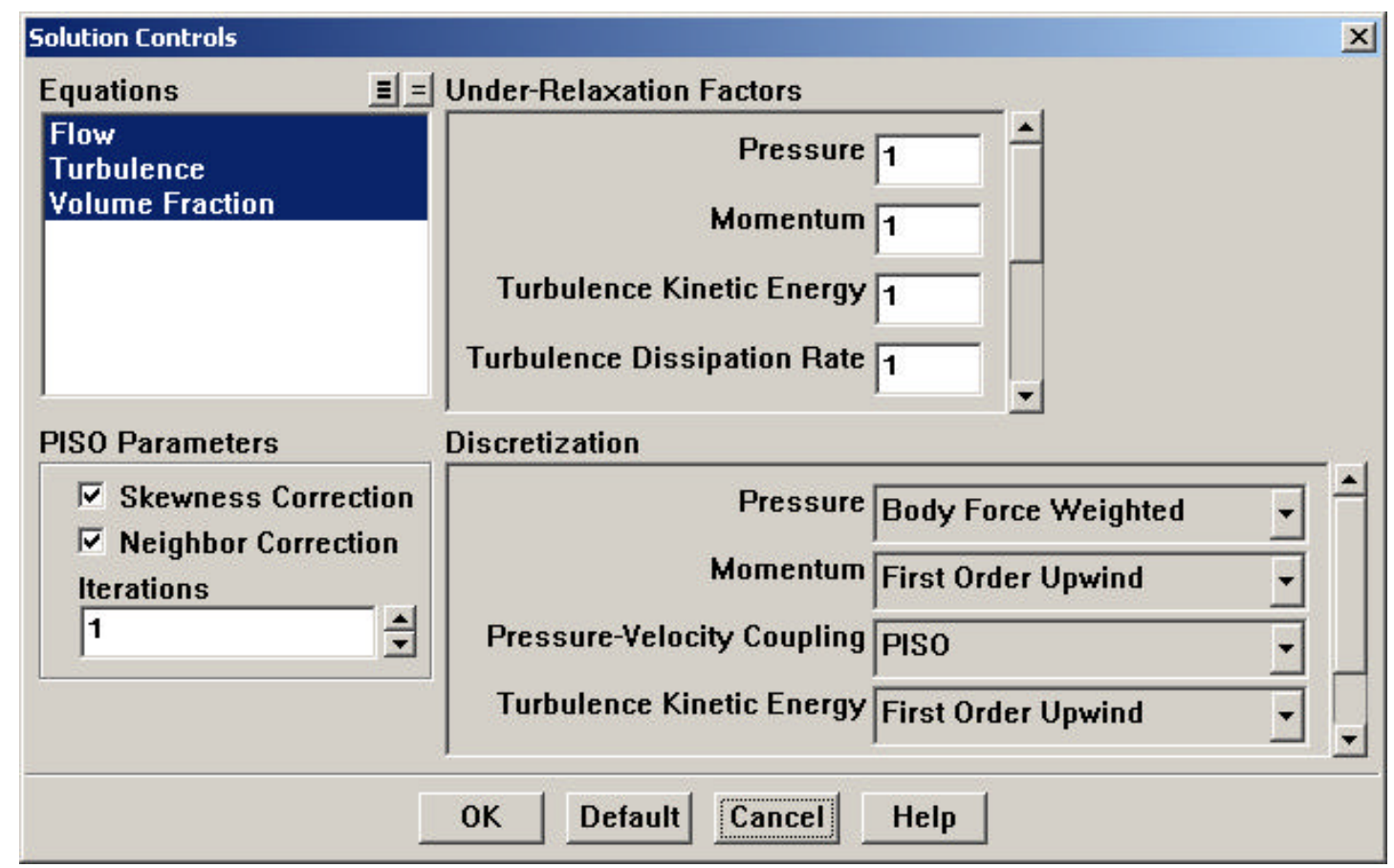

Figure 14. Solution controls panel in FLUENT

\subsection{VERIFICATION OF THE MODEL}

Once the model was developed, it has to be verified using any results presented already. The study of Sankar et al. [16] was considered for this purpose. Sankar et al. [16] studied the dynamics of liquid sloshing in elliptical road containers undergoing steady turning. The transient responses in terms of horizontal forces and vertical forces, and 
overturning moments were computed by the numerical solution of 2-D Navier-Stokes, continuity and 1-D free surface equations [16]. Sankar et al. [16] found the transient responses for two different shapes (aspect ratios) of elliptical tanks. One of those elliptical tanks was modeled using FLUENT, in this study and solved for the transient response.

An elliptical tank with a major radius (a) of 1.38395 meters and a minor radius (b) of 0.691975 meters was modeled for this purpose. All the four liquid fill levels were considered and a lateral acceleration of $0.3 \mathrm{~g}$ was given. This constant lateral acceleration simulates a steady state turn maneuver acting as the centripetal acceleration given by:

$$
a=\frac{v^{2}}{R}
$$

This model was solved for the first 8 seconds with a time step of 0.04 seconds. Dynamic coefficients of moment and horizontal force were then calculated from the output files created from FLUENT for moment, at the bottom of the tank, and horizontal force acting on the tank wall. These values are tabulated in the following table along with the values obtained by Sankar and et al [16]. A similar trend was observed in the values obtained by this study (FLUENT) and Sankar and et al [16] as well. However an exact matching of these values cannot be expected, as both the methods are basically approximations with inherent errors in the computations. There was a consistent difference of $10 \%$ between these values. 


\begin{tabular}{|c|c|c|c|}
\hline Fill Level & $\mathrm{C}_{\mathrm{m}}$ & $\mathrm{C}_{\mathrm{f}}$ & $\mathrm{K}$ \\
& FLUENT/Sankar & FLUENT/Sankar & FLUENT \\
\hline 0.3 & $1.573 / 1.354$ & $1.75314 / 1.382$ & 0.93 \\
\hline 0.5 & $1.4596 / 1.213$ & $1.51248 / 1.234$ & 1.04 \\
\hline 0.7 & $1.3138 / 1.146$ & $1.2796 / 1.158$ & 1.232 \\
\hline 0.9 & $1.0903 / 1.073$ & $1.07631 / 1.075$ & 1.63 \\
\hline
\end{tabular}

Table 1. Comparison of Dynamic coefficients calculated from FLUENT and Numerical Solution.

The results were further compared with those of Dyna-fluid models and pendulum models carried out by Salem [18]. The comparison is shown in the Figures 15, 16 and 17. The values of Dyna-fluid models and those of FLUENT fluid models were in good agreement and follow a similar trend as those of pendulum models. The pendulum models are on the conservative side and have values $20 \%$ greater than the values of Fluid models as mentioned by the author [18]. In fact the author [18] proposes to use the values of FLUENT and DYNA as reference values for the Pendulum models. Hence, the FLUENT model was found to give satisfactory results and hence is used for further analyses. 


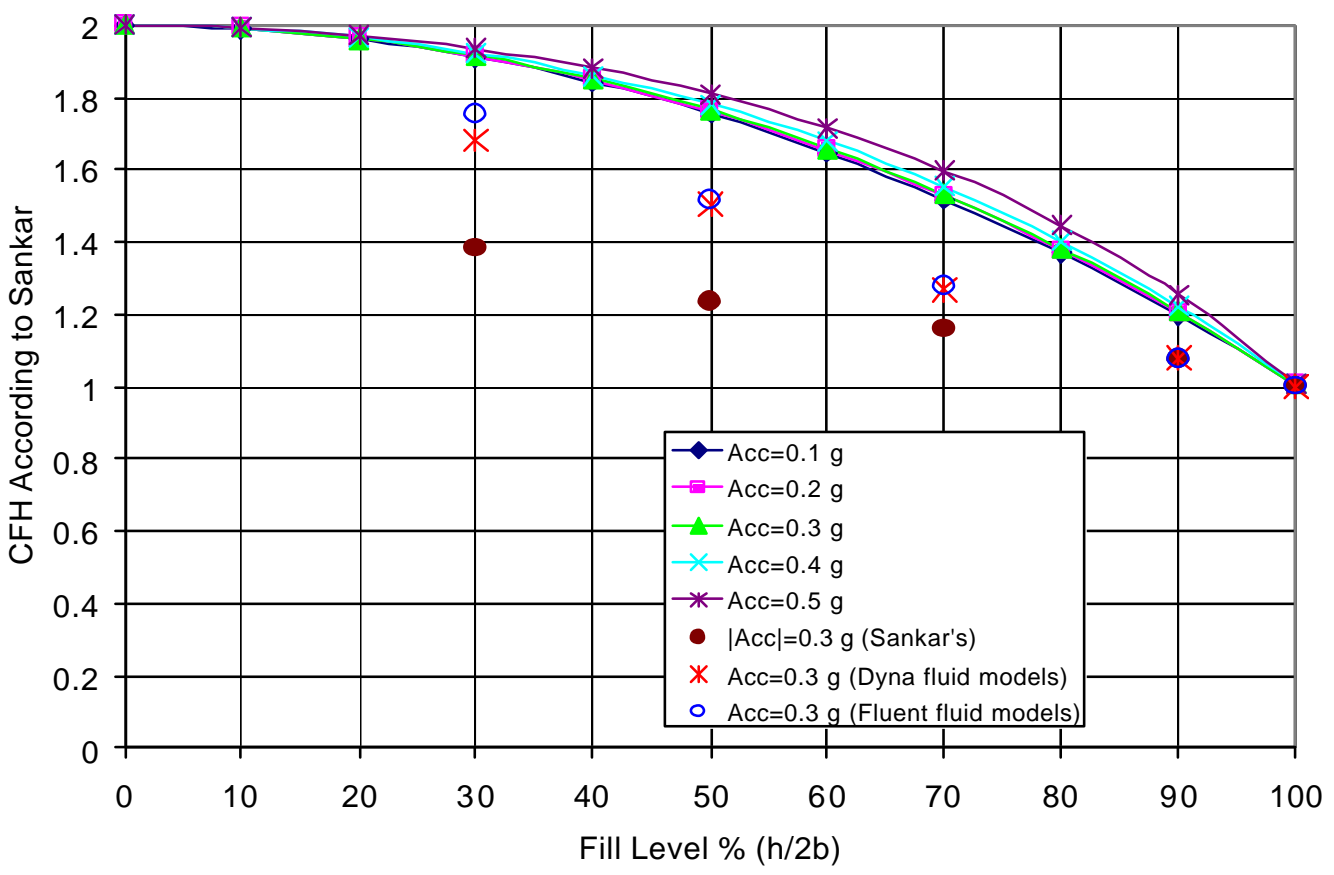

Figure 15. Horizontal force coefficients for different models

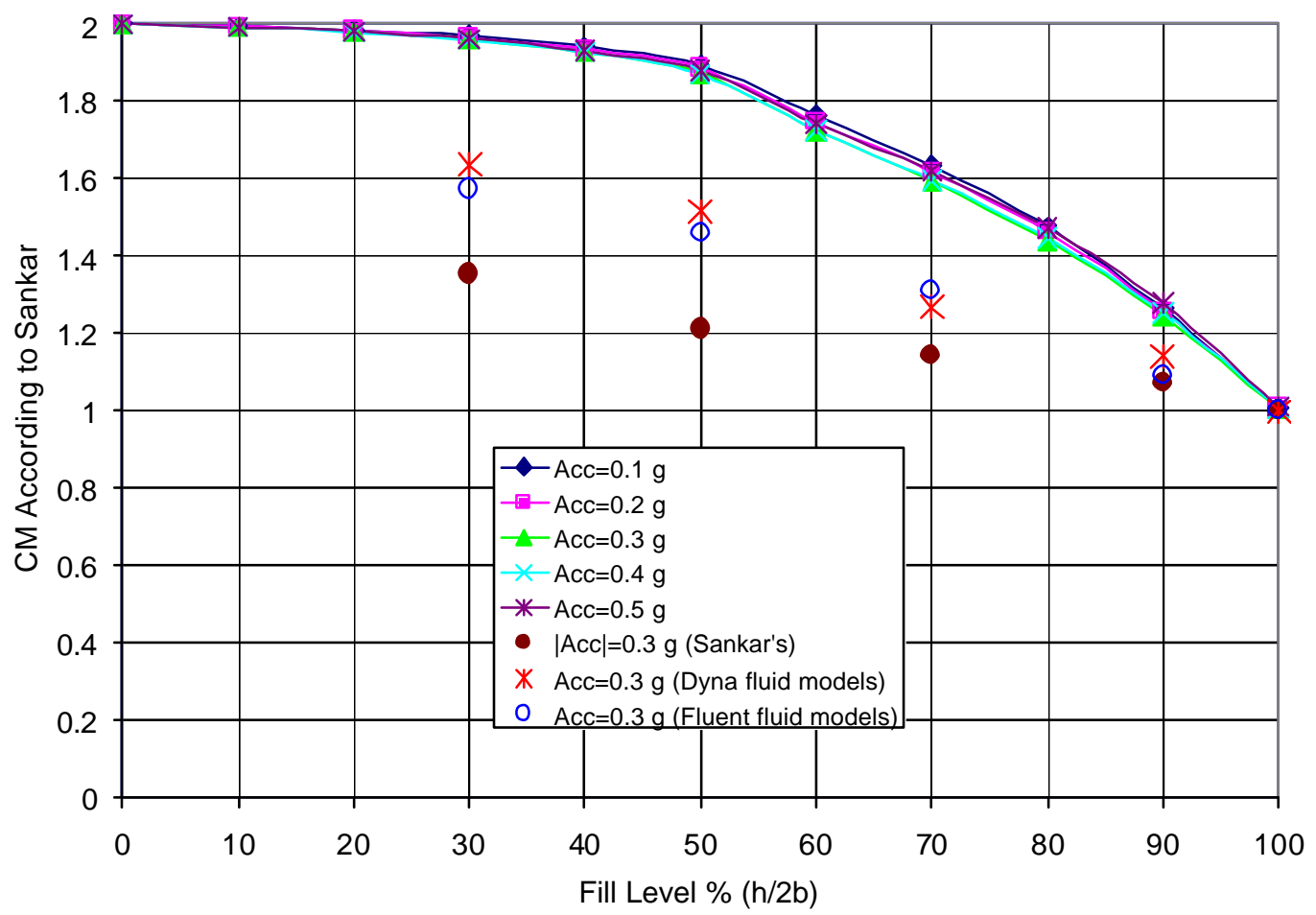

Figure 16. Moment coefficients for different models 


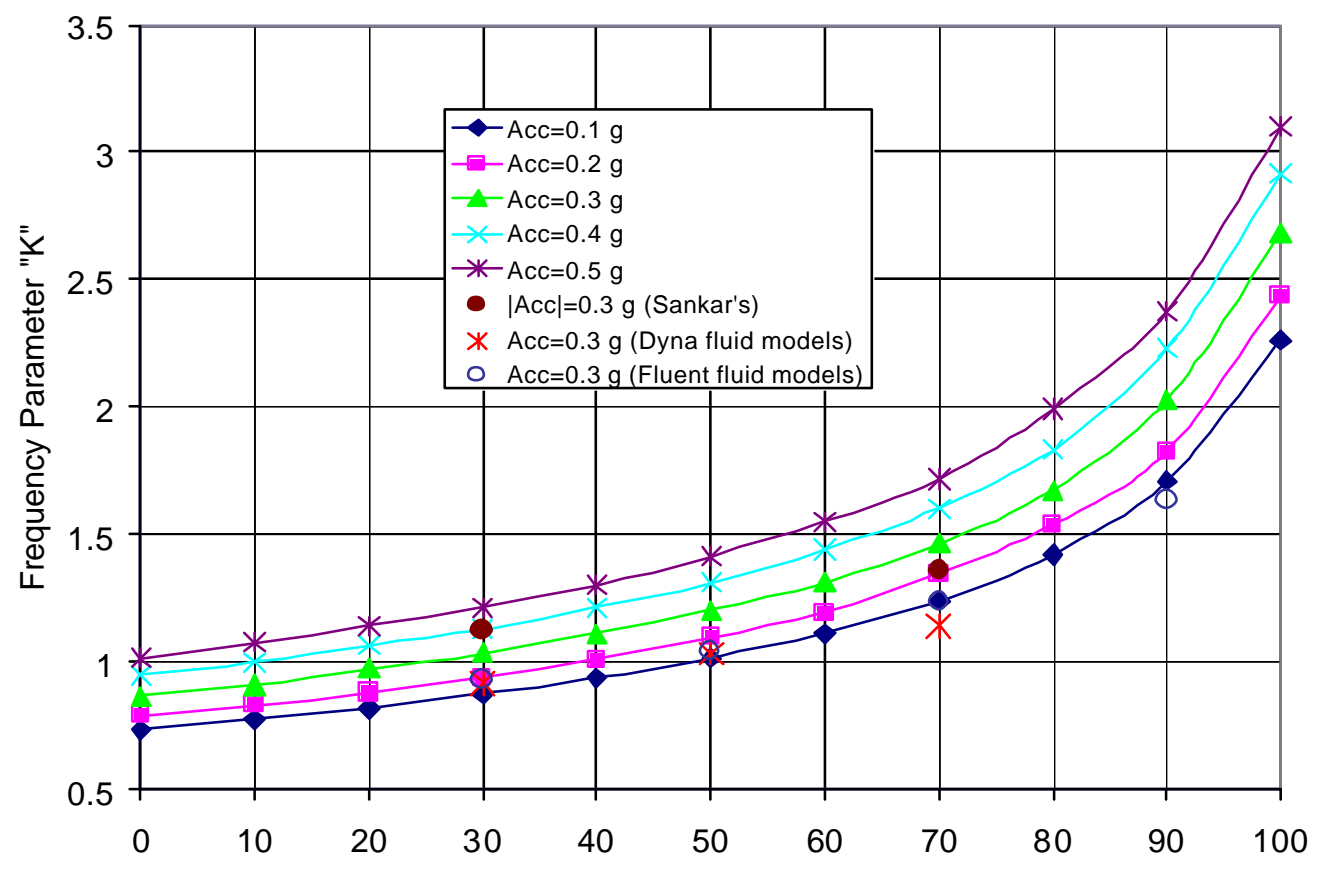

Fill Level \% (h/2b)

Figure 17. Frequency parameter "K" for different models. 


\section{CHAPTER 4}

\section{EFFECT OF KINEMATIC VISCOSITY}

\subsection{INTRODUCTION}

Viscous damping is inherent in any viscous liquid. Damping occurs because of loss of energy. If the applied forces were removed or it remained constant, then because of loss of energy during oscillations the oscillations will dampen. This damping may be significant or insignificant depending upon the kind of excitation, viscosity of the fluid, shape of the container and the amount of damping, which makes a difference in that particular application.

Aquaro [17] and Salem [18] have studied the rollover stability of partially-filled heavy-duty tanker trucks using trammel pendulums. The pendulums have to be designed properly for them to simulate the liquid sloshing accurately. The dynamics of these pendulums were investigated to determine the parameters shown in Figure 18.

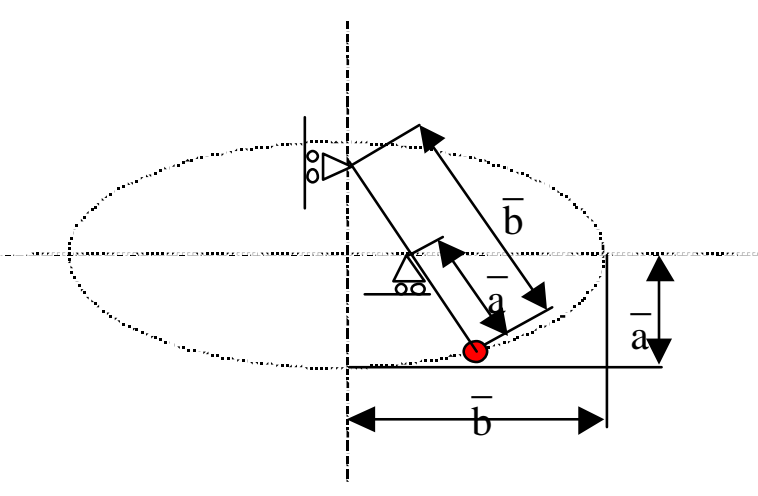

Figure 18. Center of gravity of fluid volume contour drawn by an elliptical trammel mechanism 


\subsection{PENDULUM-FLUID DYNAMIC EFFECT MATCHING}

The factors considered by Salem [18] to match the pendulum models with the dynamic effects of fluid sloshing were:

- Pendulum-fluid natural frequency matching

- Pendulum-fluid horizontal force component matching

- Pendulum-fluid static moment matching

The magnitude of sloshing was accounted for by the last two factors and the frequency of was taken care by the first factor considered. But, the pendulums models based upon the approach adopted by Salem [18] were unable to simulate the viscous damping. It was decided to study the significance of viscous damping in partially filled heavy-duty tanker trucks maneuvering a constant radius turn and TOP lane change maneuver. If a general expression could be determined for viscous damping in elliptical tanks with various aspect ratios and fill levels, and subjected to different maneuvers then, a torsional damper could be incorporated in the trammel pendulums. This would take care of the viscous damping of fluid inside the tank.

The approach was to study the effect of kinematic viscosity in elliptical tanks with different aspect ratios $(1.0,1.25,1.5$ and 2.0) and different fill levels for fluids with different viscosities in both a constant radius maneuver and a TOP lane change maneuver. The hope was that it would be possible to come up with a general expression for viscous damping as a function of the tank's aspect ratio, fill level and kinematic viscosity of the fluid. 
Five different fluids with different kinematic viscosities were chosen for the purpose. FLUENT has a database of 78 materials with the least kinematic viscosity being $7.0 \mathrm{E}-6 \mathrm{~kg} / \mathrm{m}-\mathrm{s}$ of $\mathrm{n}$-Butane. The highest viscosity was of engine oil, $1.06 \mathrm{~kg} / \mathrm{m}$ s. Hence, values of kinematic viscosities in the range from $7.0 \mathrm{E}-6 \mathrm{~kg} / \mathrm{m}-\mathrm{s}$ to 1.06 $\mathrm{kg} / \mathrm{m}$-s were considered. The following liquids were chosen as the secondary phases in the analyses carried out.

\begin{tabular}{|l|c|c|}
\hline \multicolumn{1}{|c|}{ Material } & $\begin{array}{c}\text { Viscosity } \\
(\mathrm{kg} / \mathrm{m}-\mathrm{s})\end{array}$ & $\begin{array}{c}\text { Density } \\
(\mathrm{kg} / \mathrm{m} 3)\end{array}$ \\
\hline n-Butane & $7.0 \mathrm{E}-6$ & 2.46 \\
\hline n-Heptane liquid & $4.0 \mathrm{E}-4$ & 684 \\
\hline Ethyl alcohol liquid & $1.2 \mathrm{E}-3$ & 790 \\
\hline Ethylene glycol & $1.5 \mathrm{E}-2$ & 1111.4 \\
\hline Engine oil & 1.06 & 889 \\
\hline
\end{tabular}

Table 2. Materials used in the analysis.

The liquids shown above were used for the secondary phase. Air was considered as the primary phase. Viscosity of water, $1.003 \mathrm{E}-2$ falls between the viscosities of Ethyl alcohol liquid and Ethylene glycol. Water is the fluid considered in all the analyses carried out later in this study, to establish the effects of baffles. First a circular tank (elliptical tank with elliptical ratio of 1.0) maneuvering a constant radius turn was 
considered. This was simulated by applying a constant lateral acceleration in the direction opposite to the direction of turning.

Experiments were conducted at Aberdeen Test Center (ATC) for constant radius turns and other lane change maneuvers. Constant radius turns of $75 \mathrm{ft}$ radius and $100 \mathrm{ft}$ radius were investigated. Lane change maneuvers are explained in Chapter 5. From the experiments conducted at ATC, it was found by Mohamed [18] that $0.3 \mathrm{~g}$ is the critical acceleration at which rollover was triggered. This critical acceleration of $0.3 \mathrm{~g}$ was used in

all the analyses to simulate a constant radius turn. The critical lateral acceleration of $0.3 \mathrm{~g}$ was specified in $\mathrm{x}$-direction as the gravitational acceleration in the operating conditions panel in FLUENT.

\subsection{DAMPING FACTOR}

Each fluid was analyzed individually and then the logarithmic decrement $(\delta)$ and damping factor $(\zeta)$ of the moment and horizontal force were calculated using the logarithmic decrement formulas:

$$
\begin{aligned}
& \delta=\frac{1}{n} \ln x_{0} / x_{n} \\
& \delta=2 \pi \zeta
\end{aligned}
$$


The damping factors were then calculated for each fluid and plotted in Figures 19, 20 and 21. The damping factor $(\zeta)$ is plotted on the yaxis and $\ln$ (kinematic viscosity) on the $\mathrm{x}$-axis. The damping factors were found to increase with an increase in kinematic viscosity. However, the magnitude of damping was insignificant.

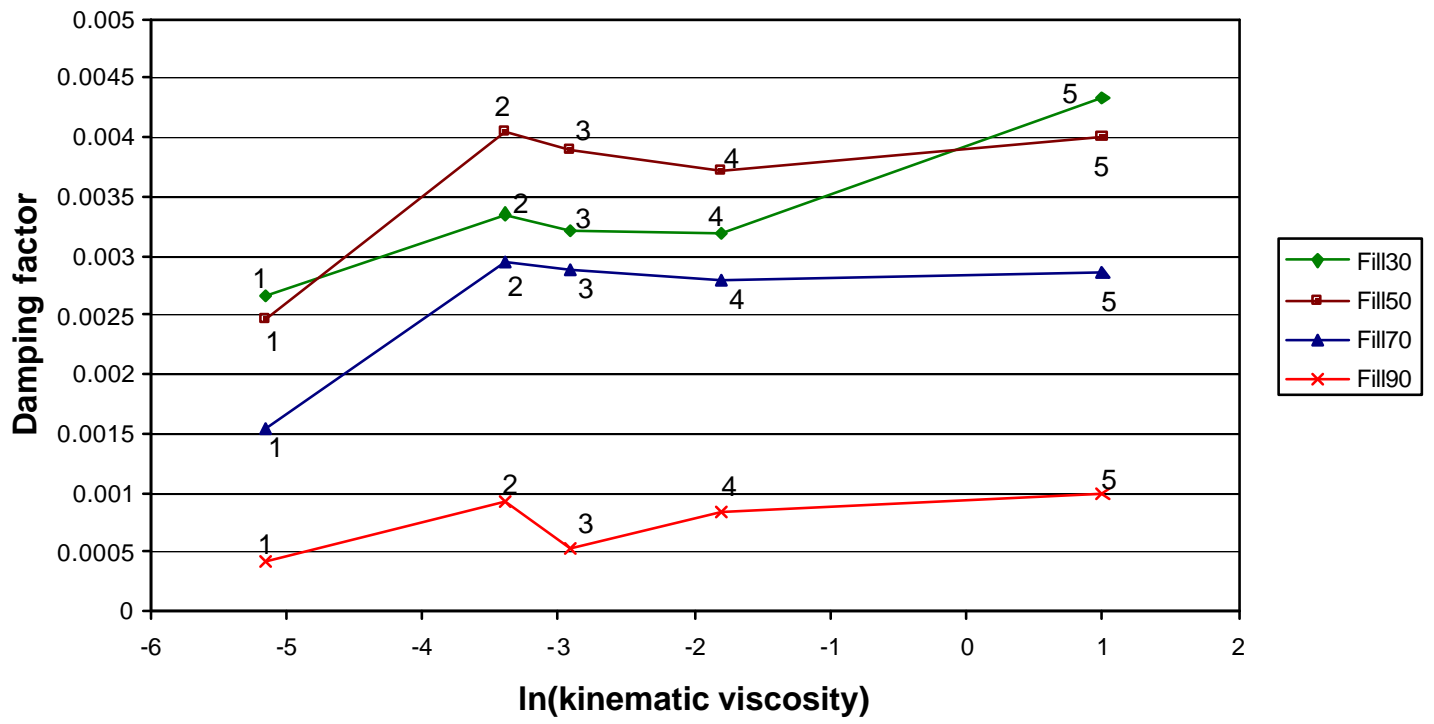

Figure 19. Damping factor (horizontal force) in a circular tank with lateral acceleration of $0.3 \mathrm{~g}$ 


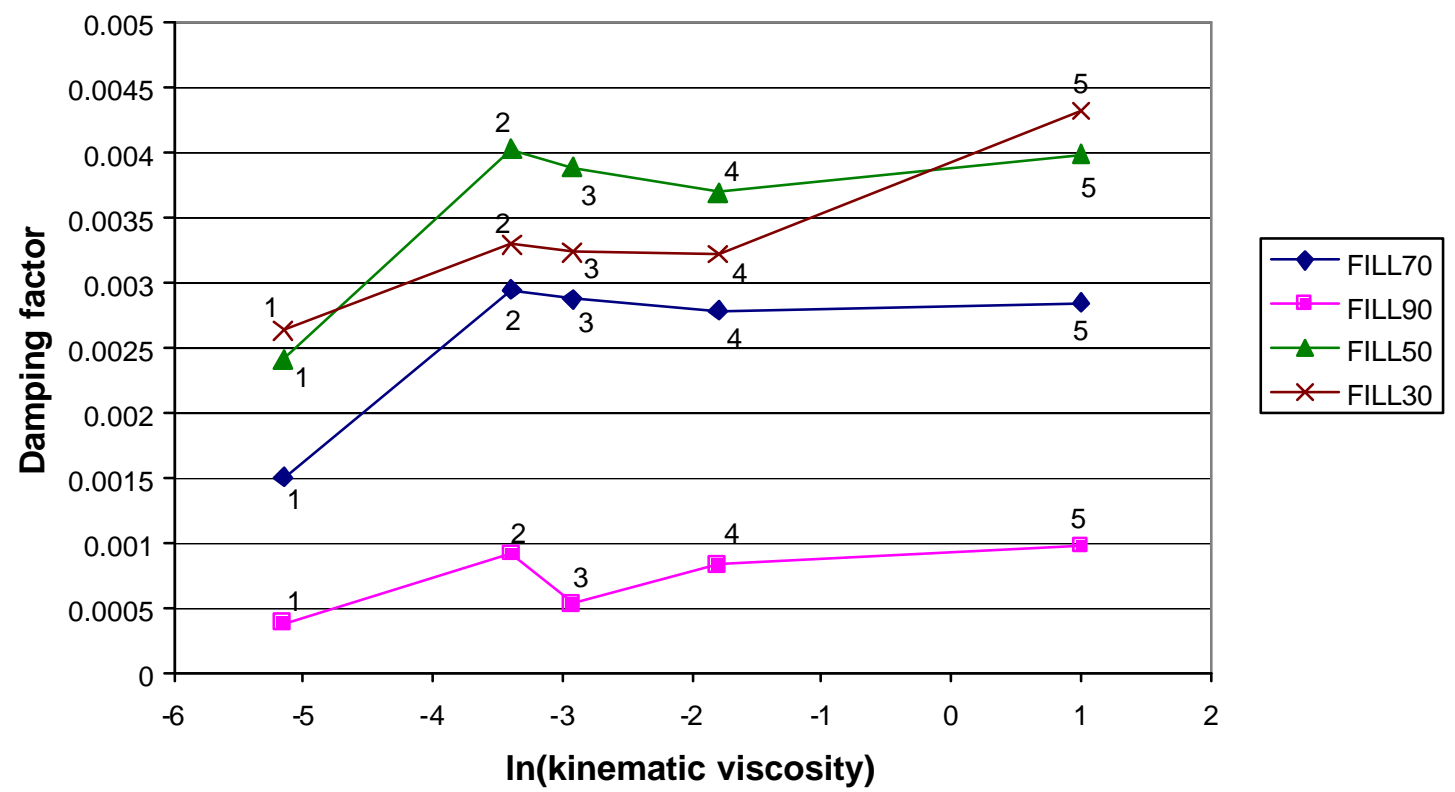

Figure 20. Damping factor (moment) in a circular tank with lateral acceleration of $0.3 \mathrm{~g}$

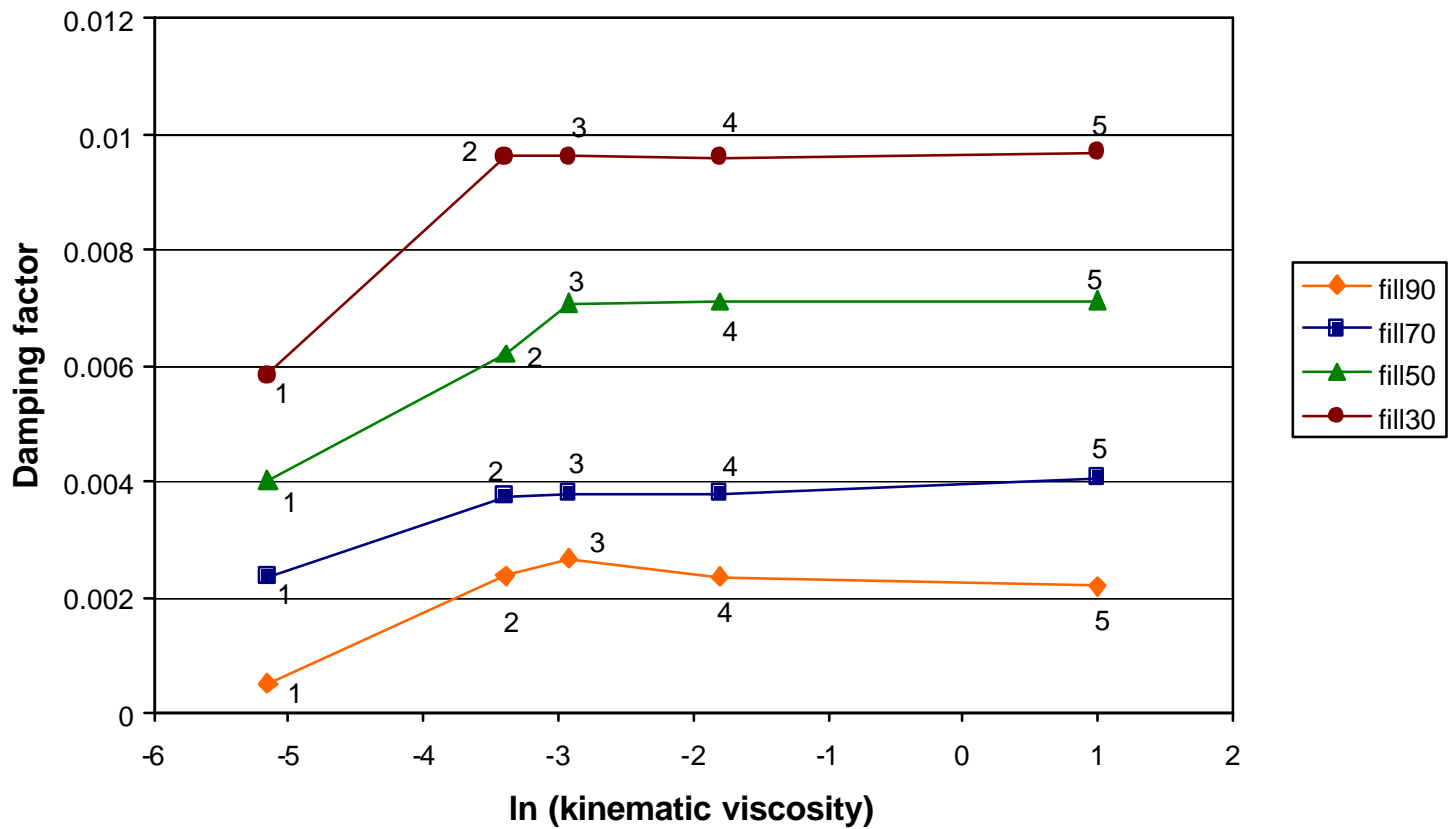

Figure 21. Damping factor (horizontal force) in an elliptical tank with lateral acceleration of $0.3 \mathrm{~g}$ 
The damping factor from moment is plotted in Figure 20. Similar analysis was carried out for an elliptical tank with aspect ratio of 2.0 with all the test fluids. Damping factor was calculated and plotted in Figure 21. It was found that the damping factors in all the cases were small in magnitude and hence, not significant.

The main intention behind these analyses was to find the characteristics of a torsional damper that may be incorporated into the trammel pendulum model so that it could simulate viscous damping of the liquid inside the tanker. The damping factor due to kinematic viscosity was found to be negligible in both circular and elliptical tanks maneuvering a constant radius turn. Hence, the effort aimed at finding viscous damping in elliptical tanks with other aspect ratios was suspended. Analyses were not carried out for TOP lane change maneuver.

Upon careful observation of Figures 19, 20 and 21 it is seen that there is an increase in the damping factor from the first point (material) to second point (material), then a slight decrease till third point, and then a steady increase till the last point. It should be remembered that each point in the plot corresponds to a different material. So, the initial increase in damping factor, and then a decrease can be attributed to other fluid properties such as density and molecular weight. It was decided to study this trend in the damping factor and hence, analyses were carried out for a circular tank maneuvering a constant radius turn. 
In this new set of analyses all the fluids that were mentioned earlier were used. Analyses were also carried out for different fluids by simply changing the Kinematic viscosities. Other properties such as density and molecular weight were kept constant, and equal to those of the first material. The results obtained from these analyses are shown in Figure 22.

There was a steady increase in the damping factors obtained from the same liquid when only the kinematic viscosities were changed unlike the first case with totally different fluids. Hence, the trend in the damping factor observed in Figures 19, 20 and 21 may be attributed to the other properties of the fluid such as, density and molecular weight. However, the damping factors were very low in this case as well.

\section{Different liquids Vs same liquid}

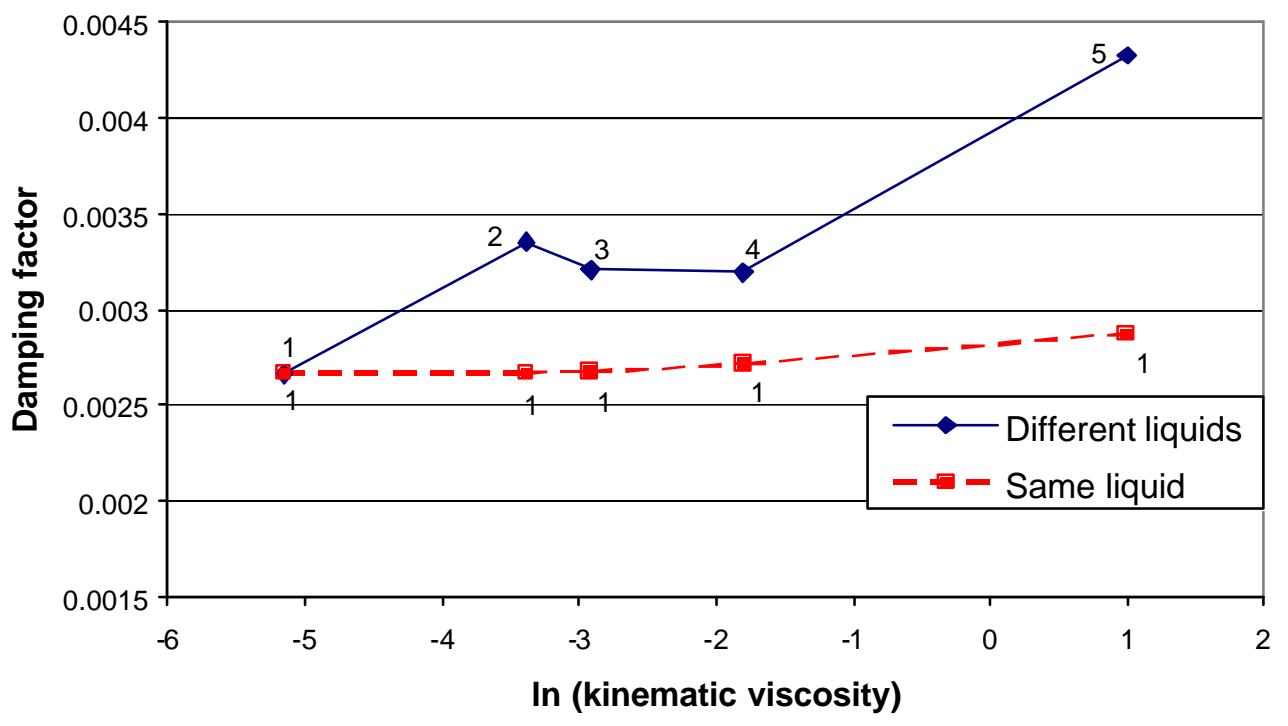

Figure 22. Comparison of damping factor for different fluids with those of same fluid with only the kinematic viscosity changed 
It may be concluded that the effect of viscous damping is negligible for the range of kinematic viscosities and maneuvers that have been considered in this study. Hence, there is no need to calculate any general expression for viscous damping in terms of tank geometry, fill level, kinematic viscosity of the fluid, and the types of maneuver considered. There is no necessity for including a torsional damper in the trammel pendulum models. 


\section{CHAPTER 5}

\section{EFFECTS OF BAFFLES}

Next step in this study was to determine the effects of different baffle designs on minimizing the lateral fluid sloshing oscillations in tankers maneuvering various paths. If the baffles considered can lower the maximum moment or the maximum force or both by a considerable amount then that would have a significant impact on increasing the rollover threshold velocity, thereby, making transportation by heavy-duty tankers safer.

\subsection{BAFFLE CONFIGURATION}

Four different kinds of baffles namely, horizontal baffles, vertical baffles, horizontal-vertical baffles and diagonal baffles were considered in this study. Configurations of various baffles are shown in Figure 23.

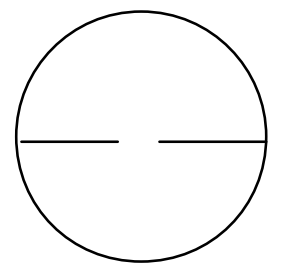

Horizontal baffles

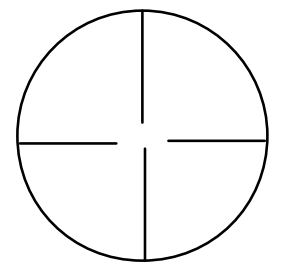

Horizontal-vertical baffles

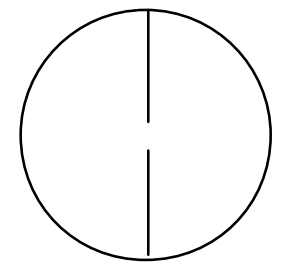

Vertical baffles

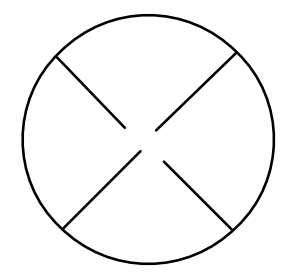

Diagonal baffles

Figure 23. Configuration of various baffles considered 
Three-dimensional tanks of $0.1 \mathrm{~m}$ long, were designed with baffles shown above for different fill levels. Also, different baffle lengths were considered. Three different 1/a ratios of $0.25,0.5$ and 0.75 were considered for this purpose. In this study, 'l' corresponds to the length of the baffle and ' $a$ ' corresponds to smallest radius of the tank. Both circular and elliptical tanks were modeled. Tanks without any baffles were also modeled. Water has been used for the fluid inside the tank in all the analyses. All of these models were then subjected to both constant radius turn and TOP lane change maneuvers. Comparison of forces and moments developed in tanks, with and without baffles, clearly illustrated the effect of various configurations of baffles. Results from these analyses are discussed below in sections on constant radius turn and TOP lane change maneuvers.

\subsection{CONSTANT RADIUS TURN}

Development of model was discussed in detail in Chapter 3. A critical constant acceleration of $0.3 \mathrm{~g}$ that simulates a constant radius turn was used for all tanks, with and without baffles. Results are discussed below for circular and elliptical tanks.

\subsubsection{CIRCULAR TANK}

A Circular tank was first analyzed without any baffles. The moment about the center point, as shown in Figure 24, and horizontal and vertical forces on the tank walls were obtained from those runs. Next, the circular tank with various configurations of baffles was analyzed, and moments and forces were obtained for each case. Dynamic coefficients of moment and force were then calculated from the values of moment and force for each case. 


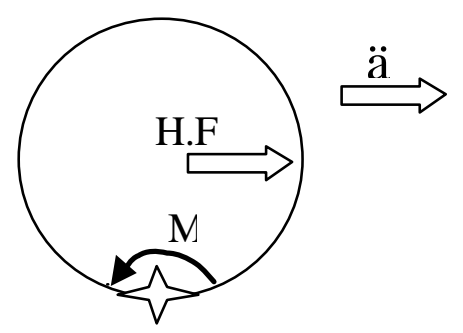

Figure 24. Moments and forces on the tank walls

Dynamic coefficient of moment is the ratio of the maximum moment to the average of the moment for the entire duration of the maneuver. Likewise dynamic coefficient of force is the ratio of the maximum force to the average of the force for the entire duration of the maneuver.

$$
\begin{gathered}
c_{m}=M_{\text {peak }} / M_{\text {mean }} \\
c_{f}=F_{\text {peak }} / F_{\text {mean }}
\end{gathered}
$$

Dynamic coefficients are an indication of the amount of sloshing present in the tanker. Once the peak moments, and dynamic coefficients of moment and force were determined for all of the cases, they were plotted against fill levels to facilitate a comparison and analysis of the effects. Each plot shows the results for a particular configuration of baffles for different $1 / \mathrm{a}$ ratios and fill levels. An $1 / \mathrm{a}$ ratio of 0.25 corresponds to shorter baffles and 1/a ratio of 0.75 represents longer baffles. An 1/a ratio of 0.0 denotes tanks without any baffles. Peak moments, and dynamic coefficients for moment and force were plotted for all the baffle configurations to elucidate the effects of various $1 /$ a ratios. 


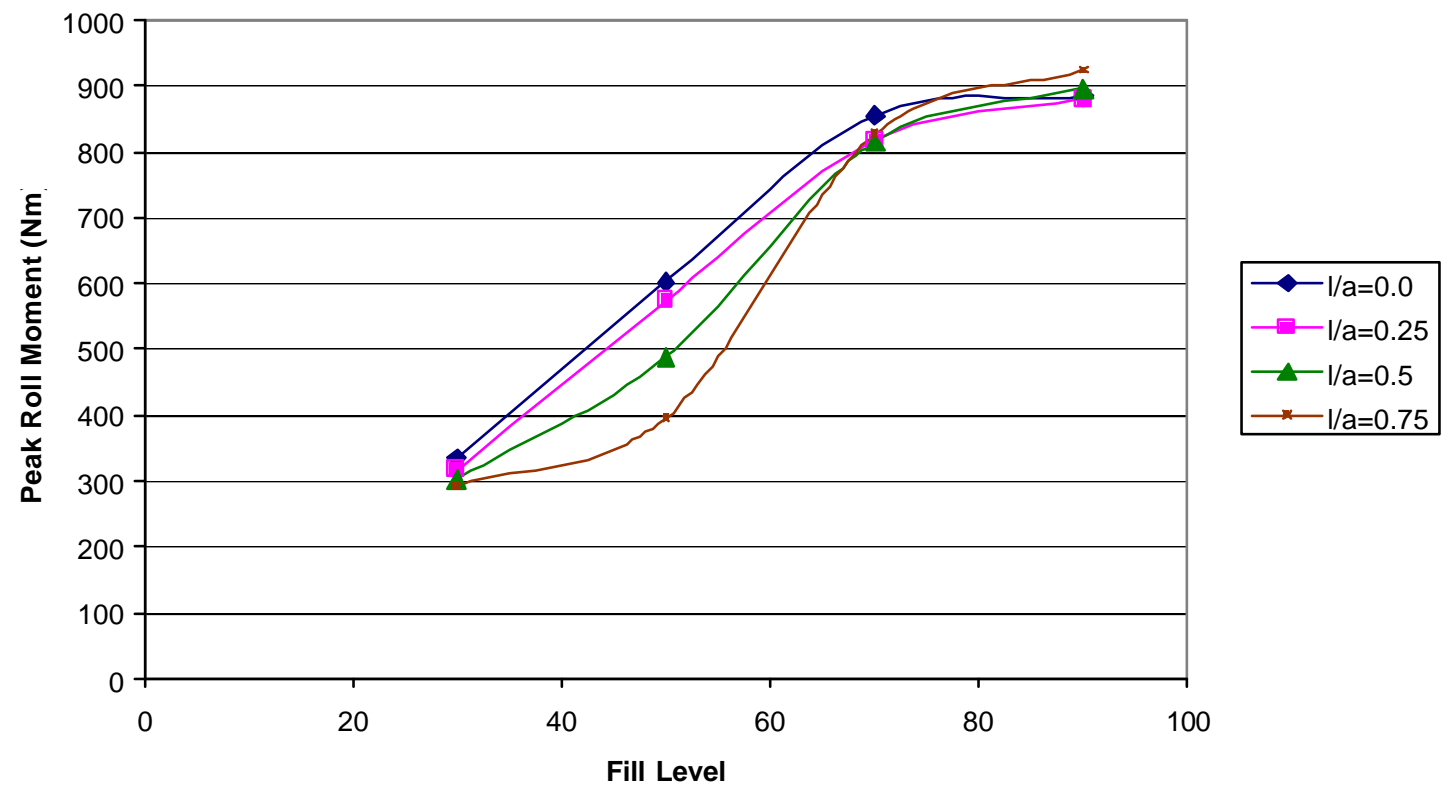

Figure 25. Peak moments for circular tank with horizontal baffles and lateral acceleration of $0.3 \mathrm{~g}$

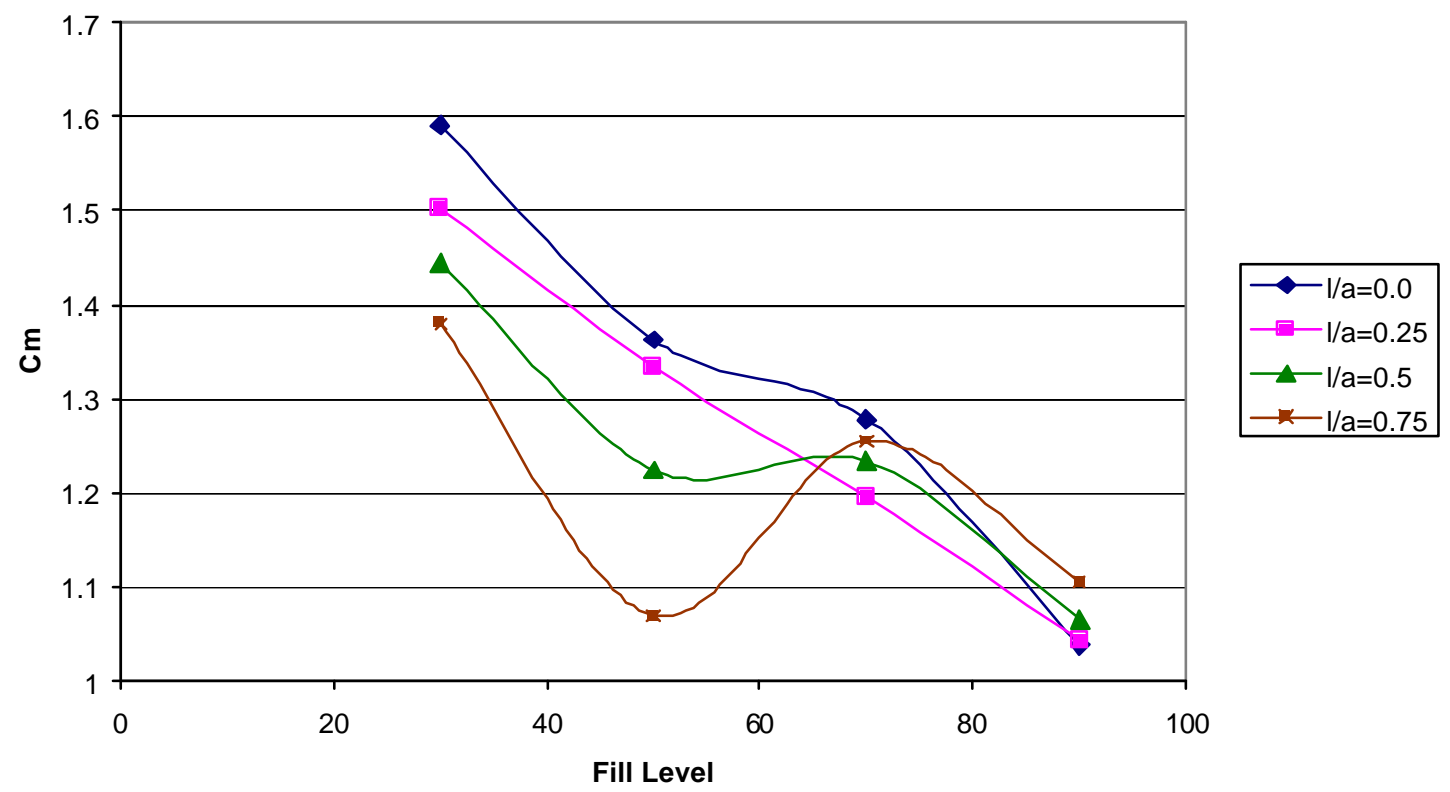

Figure $26 . \mathrm{C}_{\mathrm{m}}$ for circular tank with horizontal baffles and lateral acceleration of $0.3 \mathrm{~g}$ 


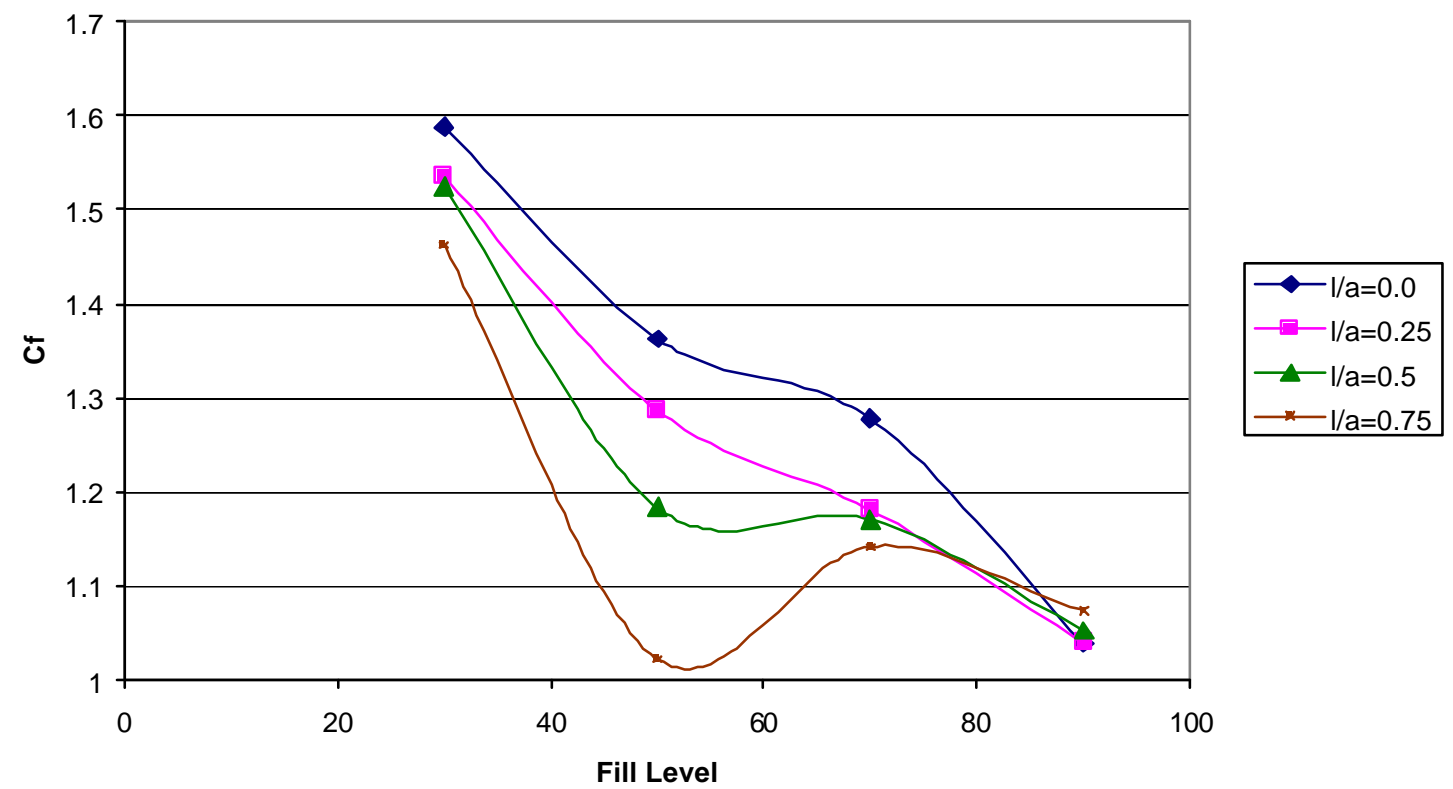

Figure 27. $\mathrm{C}_{\mathrm{f}}$ for circular tank with horizontal baffles and lateral acceleration of $0.3 \mathrm{~g}$

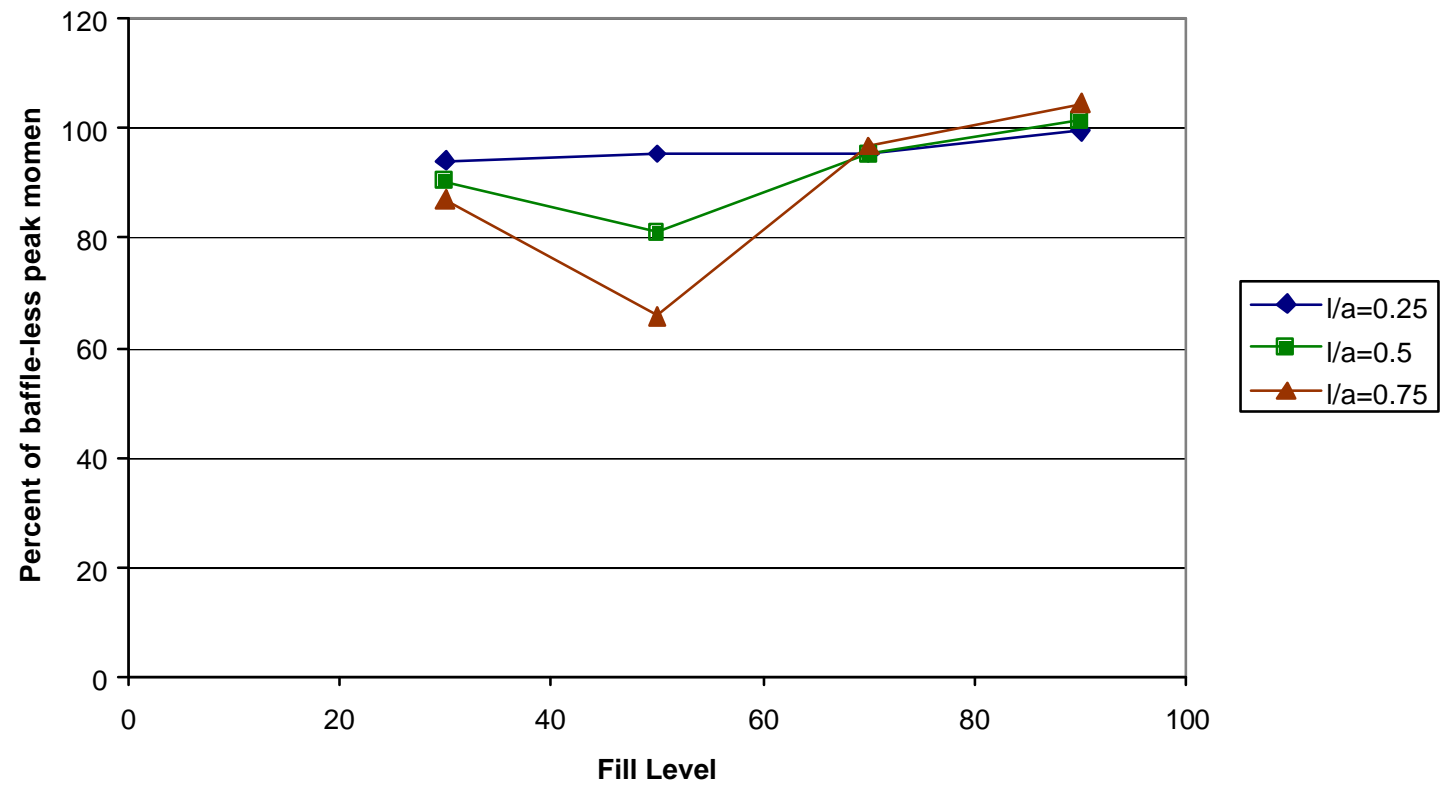

Figure 28. Percentage ratios of peak moments in a circular tank with horizontal baffles to peak moments in a circular tank without any baffles 
The Figures 25, 26 and 27 show that the horizontal baffles are effective in reducing the sloshing and moments in a circular tank maneuvering a constant radius turn. The 1/a ratio of 0.75 appeared to be more effective in reducing both sloshing and moments up to fill levels of $70 \%$. For fill levels greater than $70 \%, 1 /$ a ratio of 0.25 was the most effective. There was little discernable difference for fill level of $90 \%$ for all the three baffle lengths. This can be attributed to the limited space available, at this fill level, for any sloshing to occur. It should also be noted that the baffles were totally immersed under the fluid. There was no damping provided to the oscillations at the free surface.

The maximum reduction offered by horizontal baffles was $34 \%$ in peak moment for fill level of $50 \%$ with baffles of $1 /$ a ratio $=0.75$. Reduction at $50 \%$ fill level is more in all cases. This could be attributed to the presence of baffles at the surface. Damping provided at the free surface of the fluid is more effective than any other damping. Figure 28 shows the percentage ratios of peak moments for all the three baffle lengths to peak moments in a baffle-less tanker for each fill level.

Figure 28 shows that, at fill levels greater than $70 \%$ peak moments were actually increasing with the length of the baffles. For these fill levels greater than $70 \%$, the baffles are well under the surface of the fluid and are actually acting as blades of a turbine. At this position, under the surface of the fluid they were not able to suppress any sloshing at the surface of the fluid. This can be seen in Figures 29 and 30. Figure 29 shows the volume fraction of water in a $30 \%$ fill tank at 0.8 seconds where the fluid hits the baffles 
and comes back. Figure 30 shows volume fraction fluid for a $70 \%$ fill tank. Here, baffles act as turbine blades and increase the moments acting on the tank

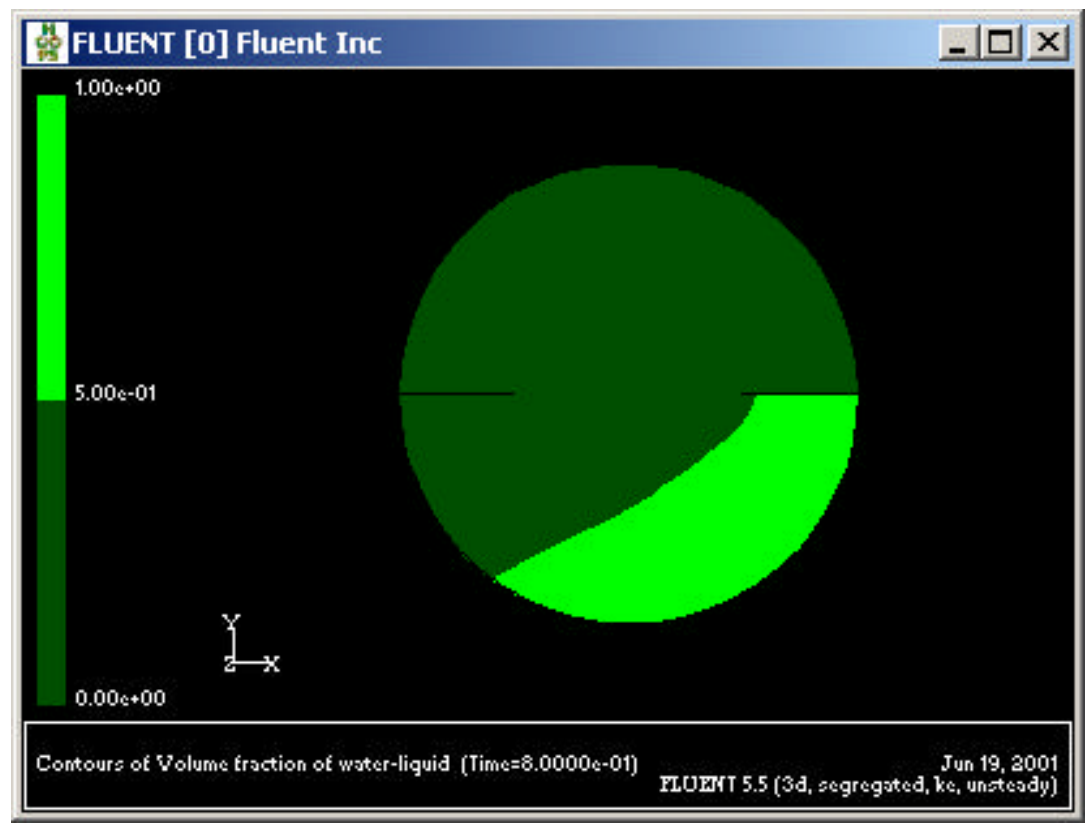

Figure 29. Volume fraction of fluid in a $30 \%$ fill tank with horizontal baffles of $1 / \mathrm{a}$ ratio $=0.5$ at 0.8 seconds

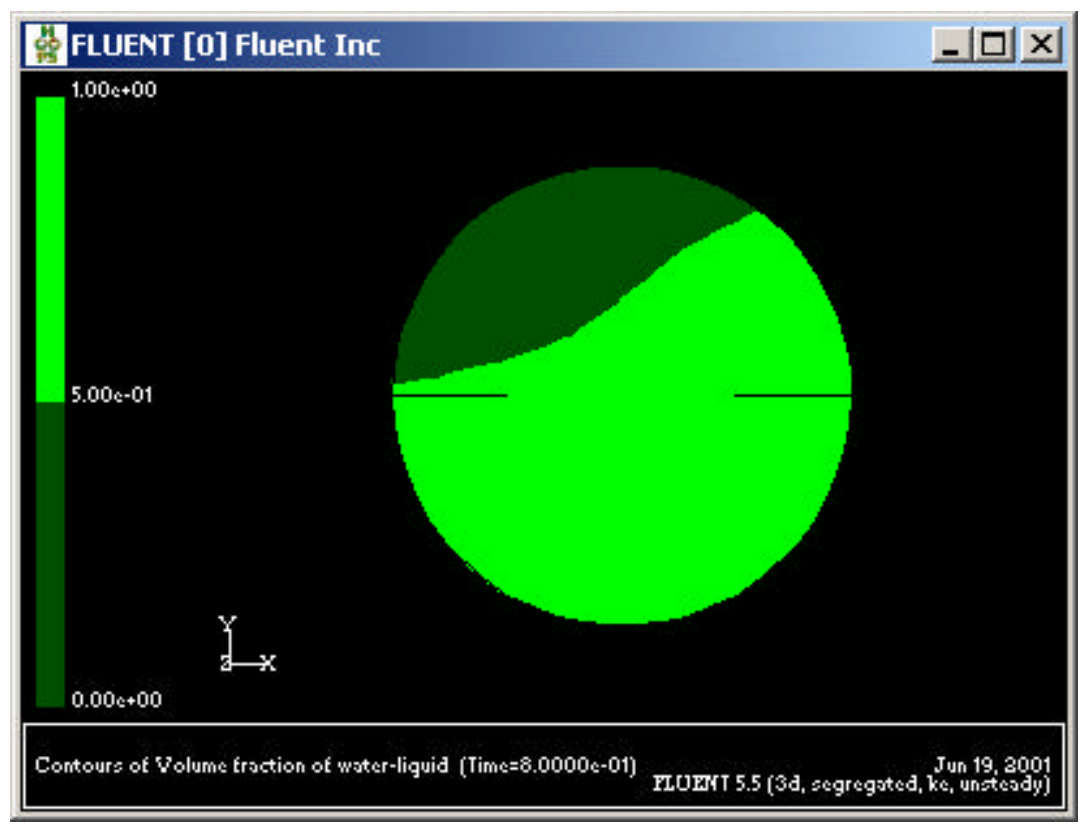

Figure 30. Volume fraction of fluid in a $70 \%$ fill tank with horizontal baffles of $1 / \mathrm{a}$ ratio $=0.5$ at 0.8 seconds. 


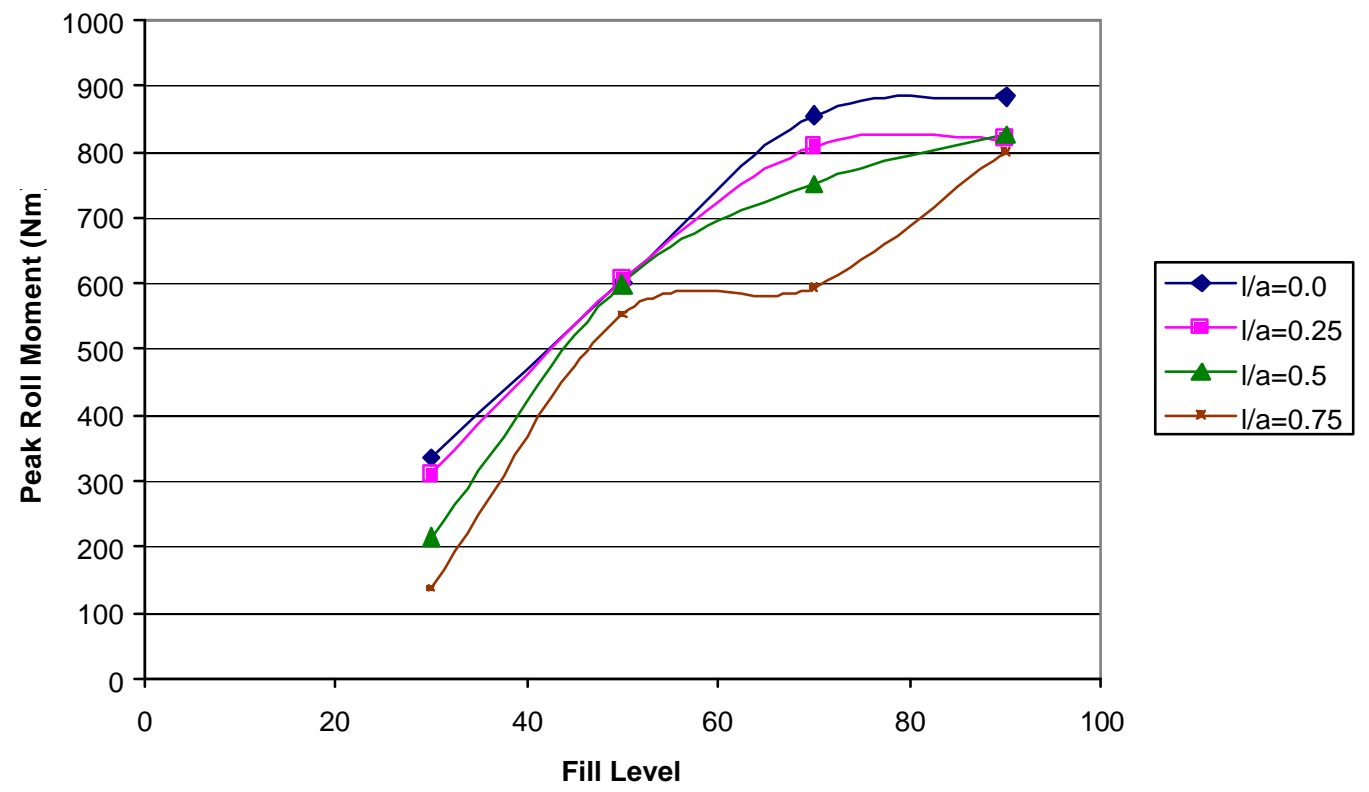

Figure 31. Peak moments for circular tank with vertical baffles and lateral acceleration of $0.3 \mathrm{~g}$

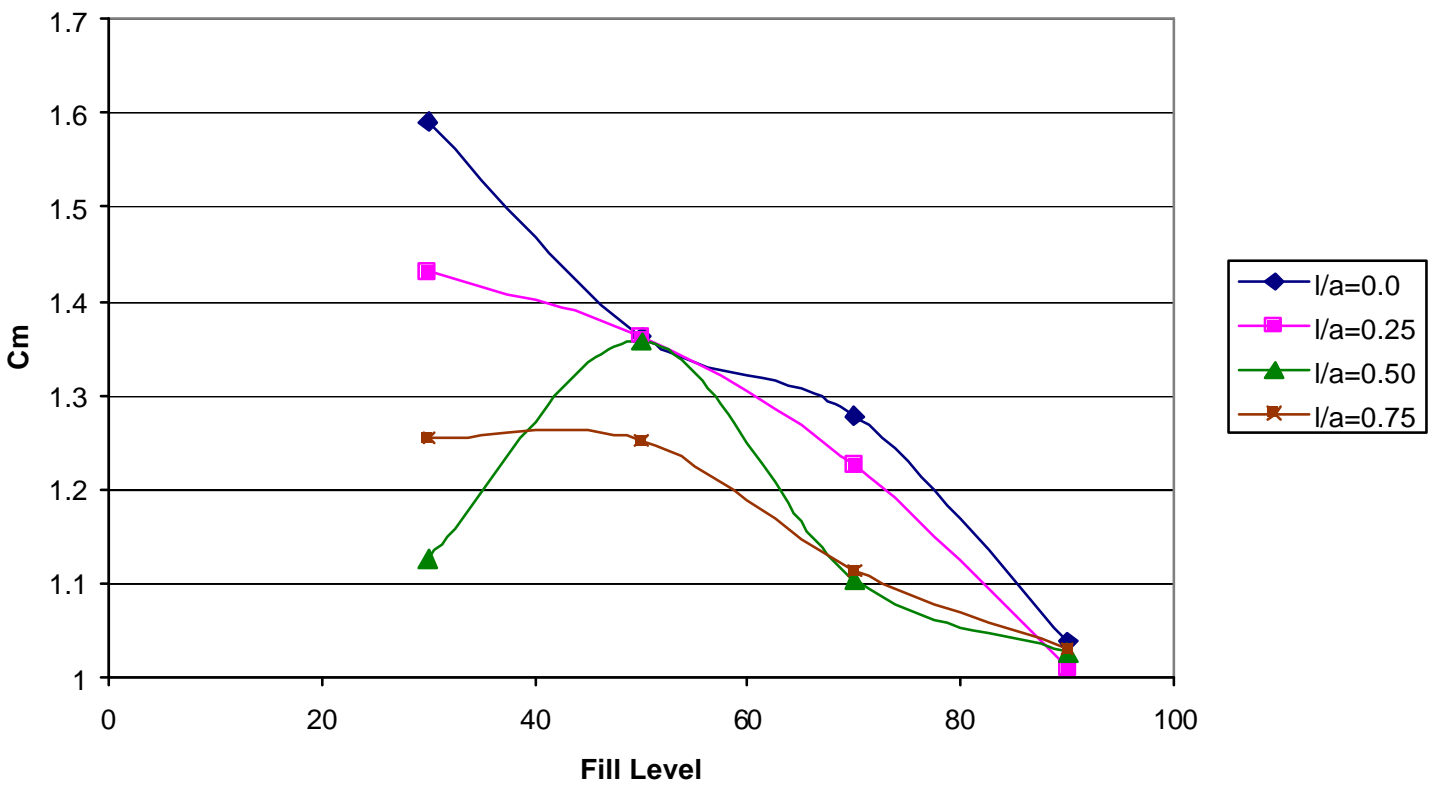

Figure 32. $\mathrm{C}_{\mathrm{m}}$ for circular tank with vertical baffles and lateral acceleration of $0.3 \mathrm{~g}$ 


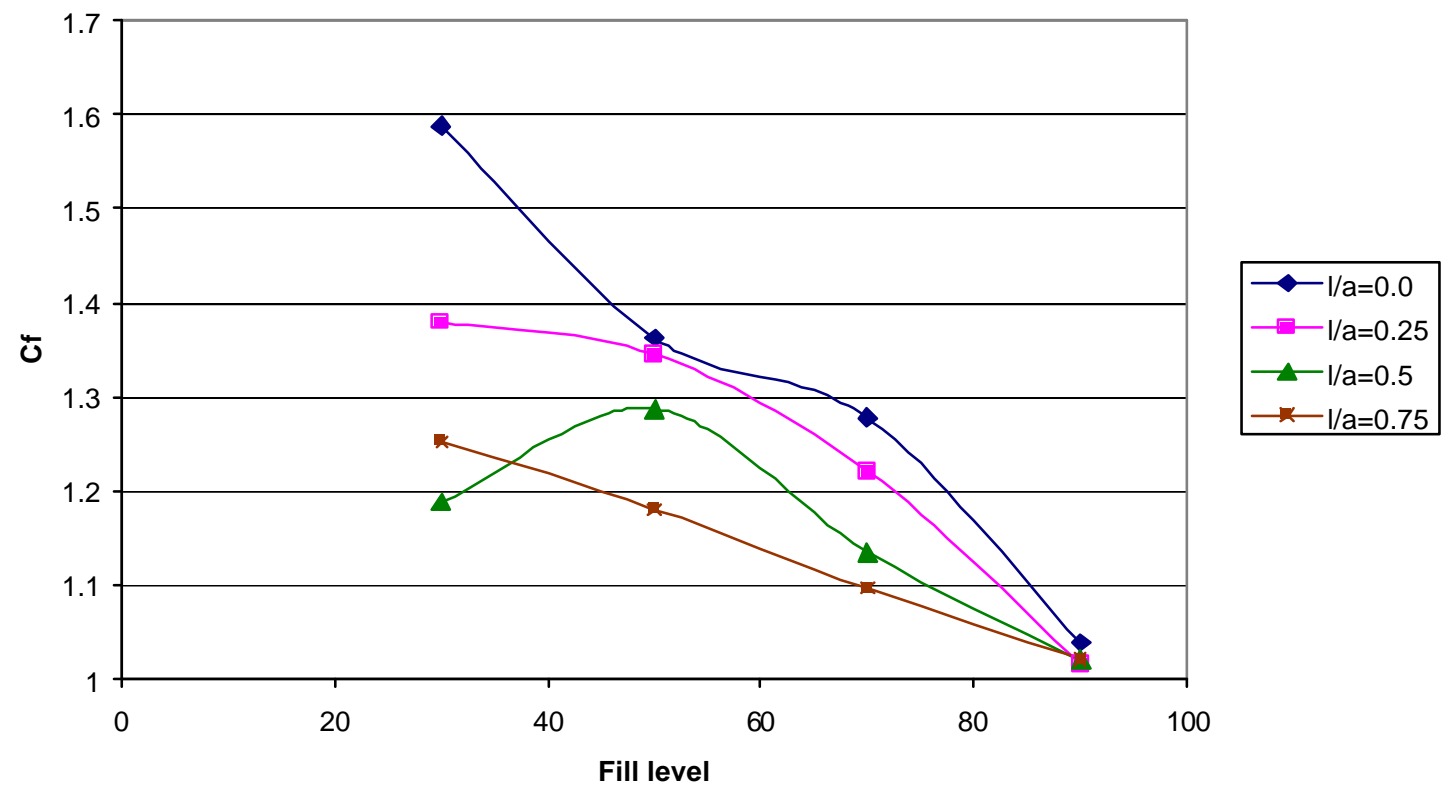

Figure 33. $\mathrm{C}_{\mathrm{f}}$ for circular tank with vertical baffles and lateral acceleration of $0.3 \mathrm{~g}$

From figures 31,32 and 33 vertical baffles appeared to be more effective than horizontal baffles at reducing the moments. The $1 / \mathrm{a}$ ratio of 0.75 reduced the moments more effectively than other of baffle lengths. This was observed to be true for all fill levels. The 1/a ratio of 0.5 also reduced the moments and sloshing, but was less effective than an $1 / \mathrm{a}$ ratio of 0.75 . Further, the $1 / \mathrm{a}$ ratio of 0.25 is less effective than the $1 / \mathrm{a}$ ratio of 0.5. It appeared that, greater the length in case of vertical baffles the more effective they were at reducing moments. Reductions of $59 \%$ in peak moments for $30 \%$ fill level, and $30 \%$ reduction for fill level of $70 \%$ were obtained using vertical baffles with $1 / a$ ratio of 0.75. Figure 34 shows the percentage ratios of peak moments for all the three baffle lengths to peak moments in a baffle-less tanker, for each fill level. Next, horizontalvertical baffles were considered. 


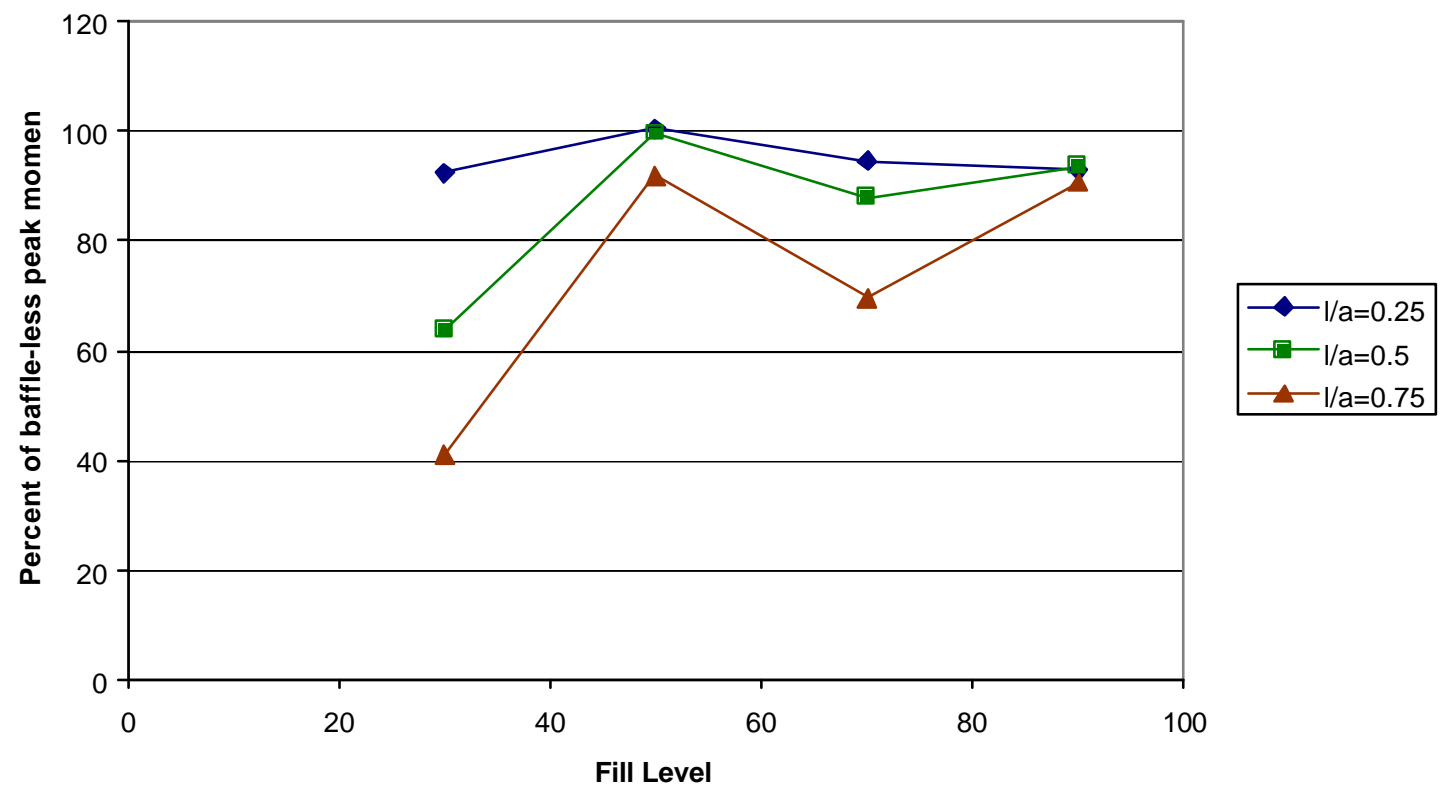

Figure 34. Percentage ratios of peak moments in a circular tank with vertical baffles to peak moments in a circular tank without any baffles

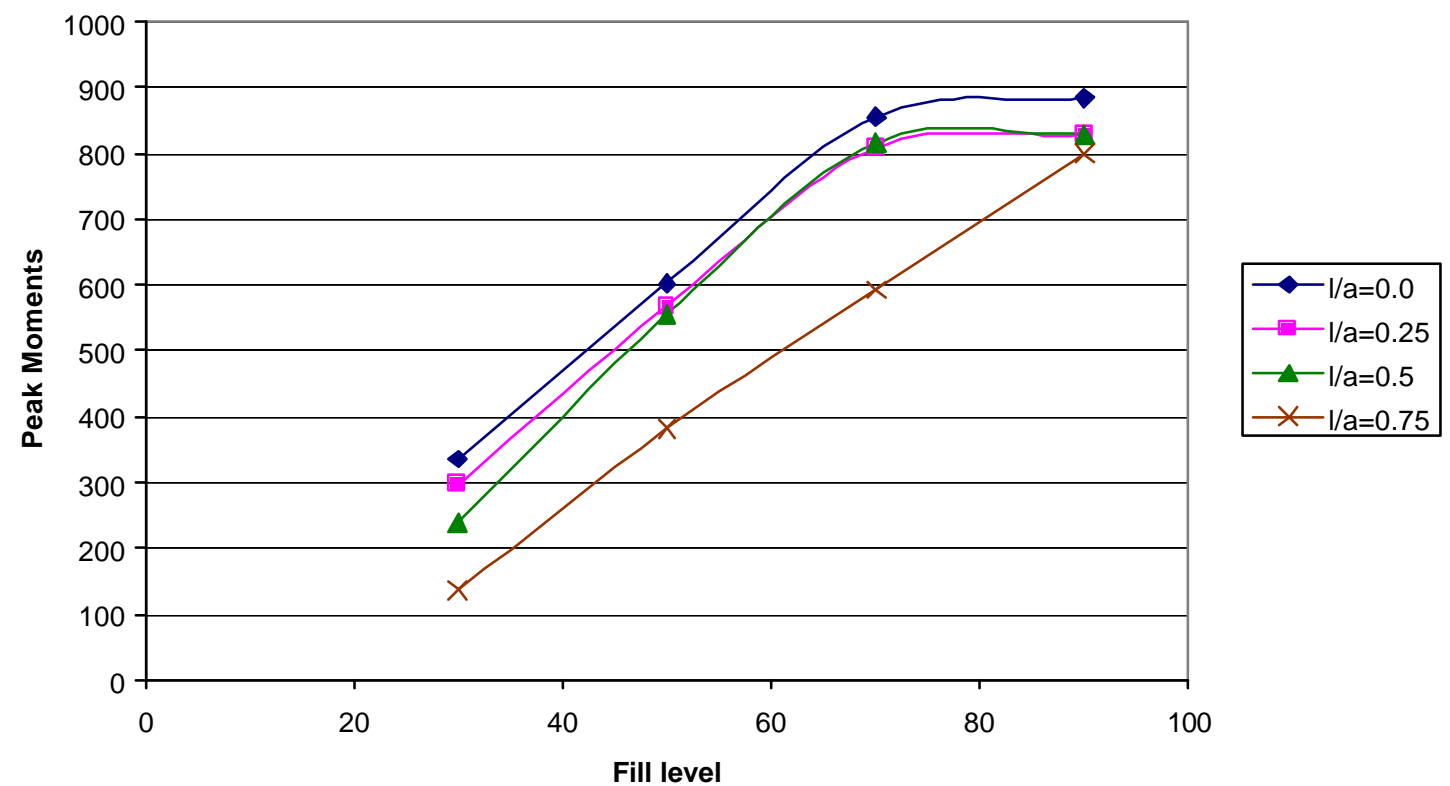

Figure 35. Peak moments for circular tank with lateral acceleration of $0.3 \mathrm{~g}$ and horizontal-vertical baffles 


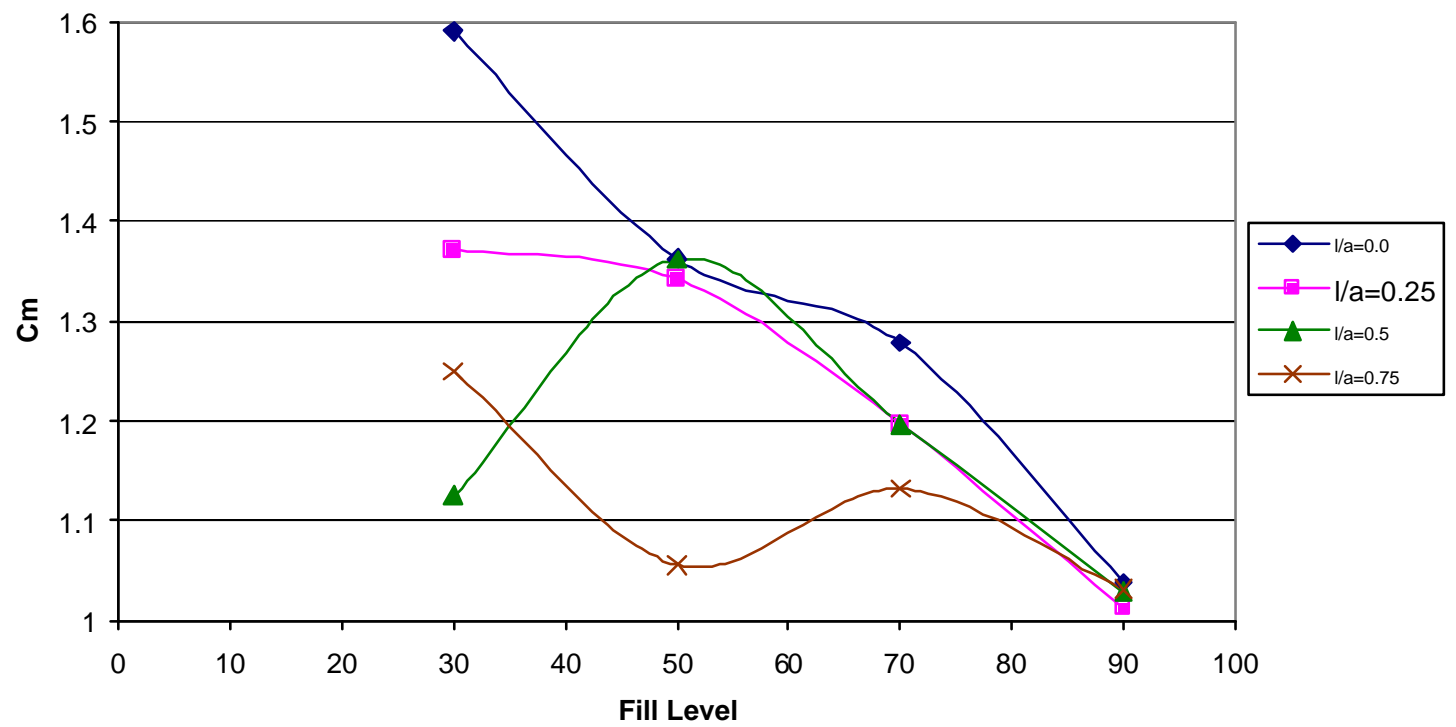

Figure $36 . \mathrm{C}_{\mathrm{m}}$ for circular tank with lateral acceleration of $0.3 \mathrm{~g}$ and horizontal-vertical baffles

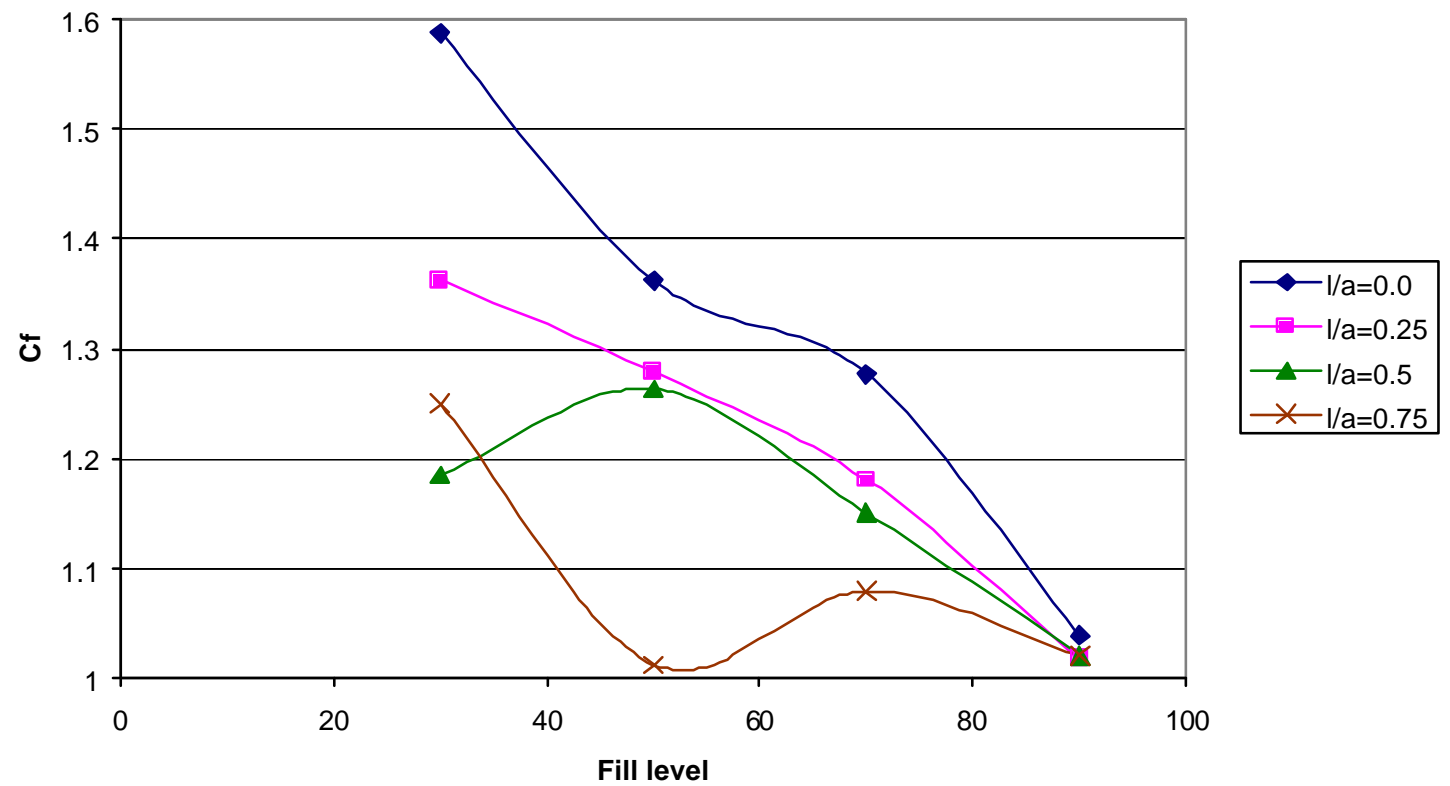

Figure $37 . \mathrm{C}_{\mathrm{f}}$ for circular tank with lateral acceleration of $0.3 \mathrm{~g}$ and horizontal-vertical baffles 
The horizontal-vertical baffles considered above in Figures 35, 36 and 37, with the added effect of both horizontal baffles and vertical baffles appeared very effective with a significant reduction in the peak moments and sloshing as well, compared to the baffles (horizontal baffles or vertical baffles) studied previously. The 1/a ratio of 0.75 was considered to be optimal for all fill levels except for fill $30 \%$ where sloshing was reduced more by an 1/a ratio of 0.5 . But, peak moment was again less for the case with $1 / a$ ratio of 0.75. A reduction of $59.2 \%$ was obtained for fill $30 \%$ with $1 / a$ ratio of 0.75 baffles. A reduction of $30 \%$ was obtained for fill levels $50 \%$ and $70 \%$. A fill level of $90 \%$ had a reduction of $9 \%$ in the peak moments. In summary, horizontal-vertical baffles were found to be more effective, when all the fill levels are considered, in reducing the peak moments, than the horizontal baffles or vertical baffles individually. Diagonal baffles are the next configuration.

It is evident from Figures 38, 39 and 40 that the values of peak moments and both the dynamic coefficients are lower in tanks with diagonal baffles compared to values in tanks without any baffles. Sloshing, which is indicated by the dynamic coefficients, is reduced more by baffles of $1 / a=0.5$ at all the fill levels except for $50 \%$ fill level, where $1 / \mathrm{a}=0.75$ was found to be more effective. More importantly, looking at the peak moments plot, $1 / \mathrm{a}=0.75$ reduced the moments, in all cases to a greater extent than other baffles. A significant reduction of $66 \%$ was obtained in the peak moment for fill level of $30 \%$ with diagonal baffles of $1 / \mathrm{a}=0.75$. Similarly a $34 \%$ reduction was seen for fill levels of $70 \%$. Figures 41 and 42 show the percentage ratios of peak moments for all the three baffle lengths (horizontal-vertical and diagonal baffles respectively) to peak moments in a baffle-less tanker for each fill level. 


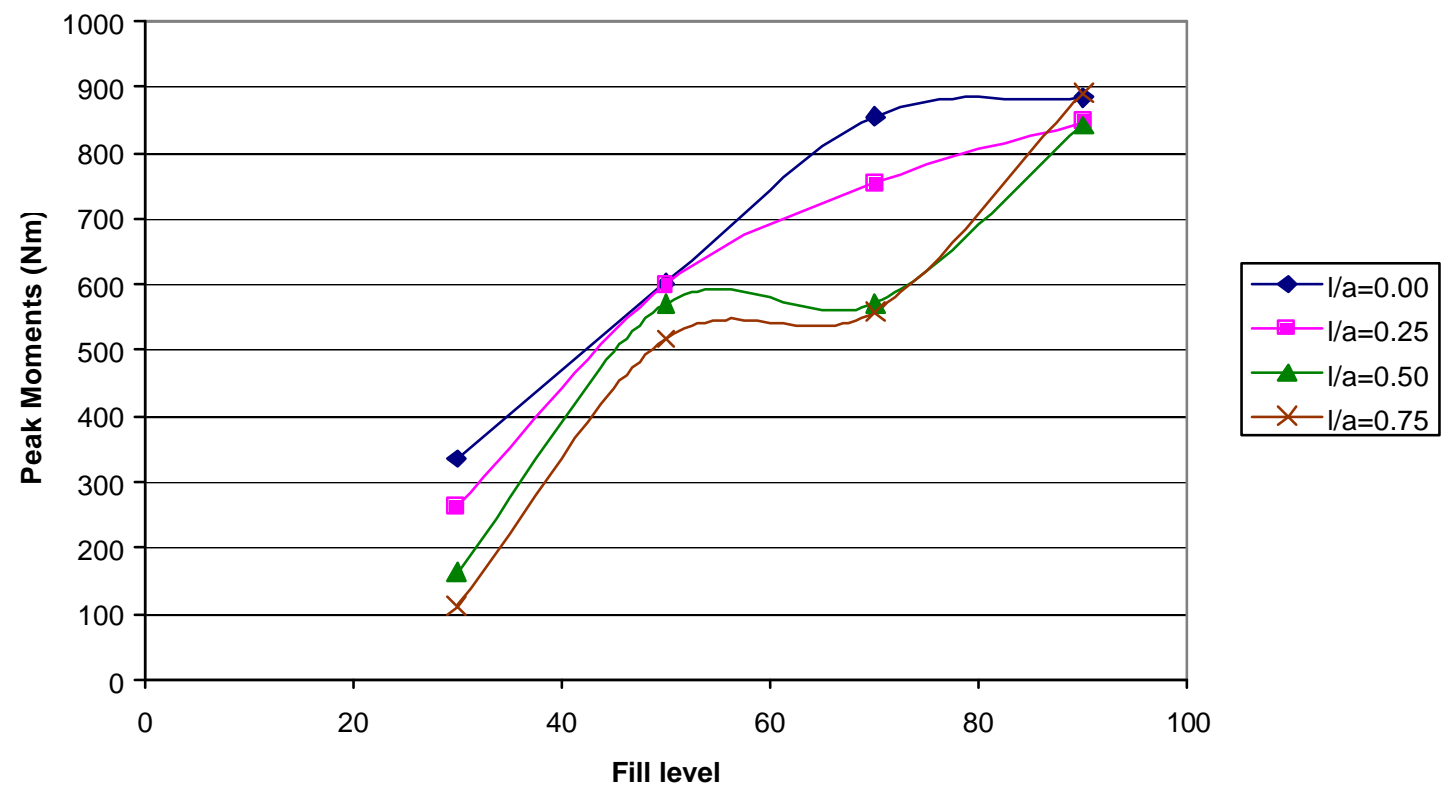

Figure 38. Peak moments for circular tank with diagonal baffles and lateral acceleration

$$
\text { of } 0.3 \mathrm{~g}
$$

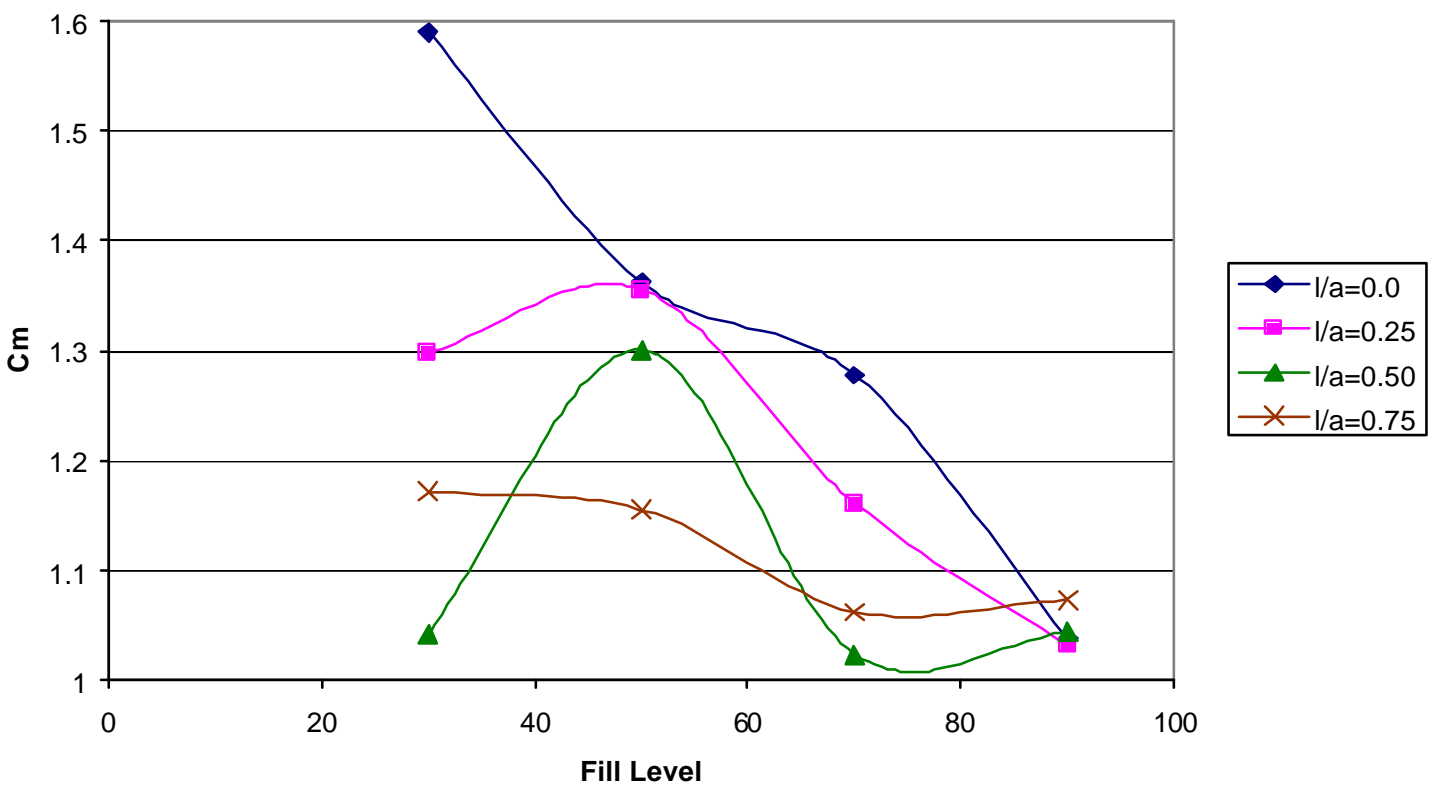

Figure 39. $\mathrm{C}_{\mathrm{m}}$ for circular tank with lateral acceleration $=0.3 \mathrm{~g}$ and diagonal baffles 


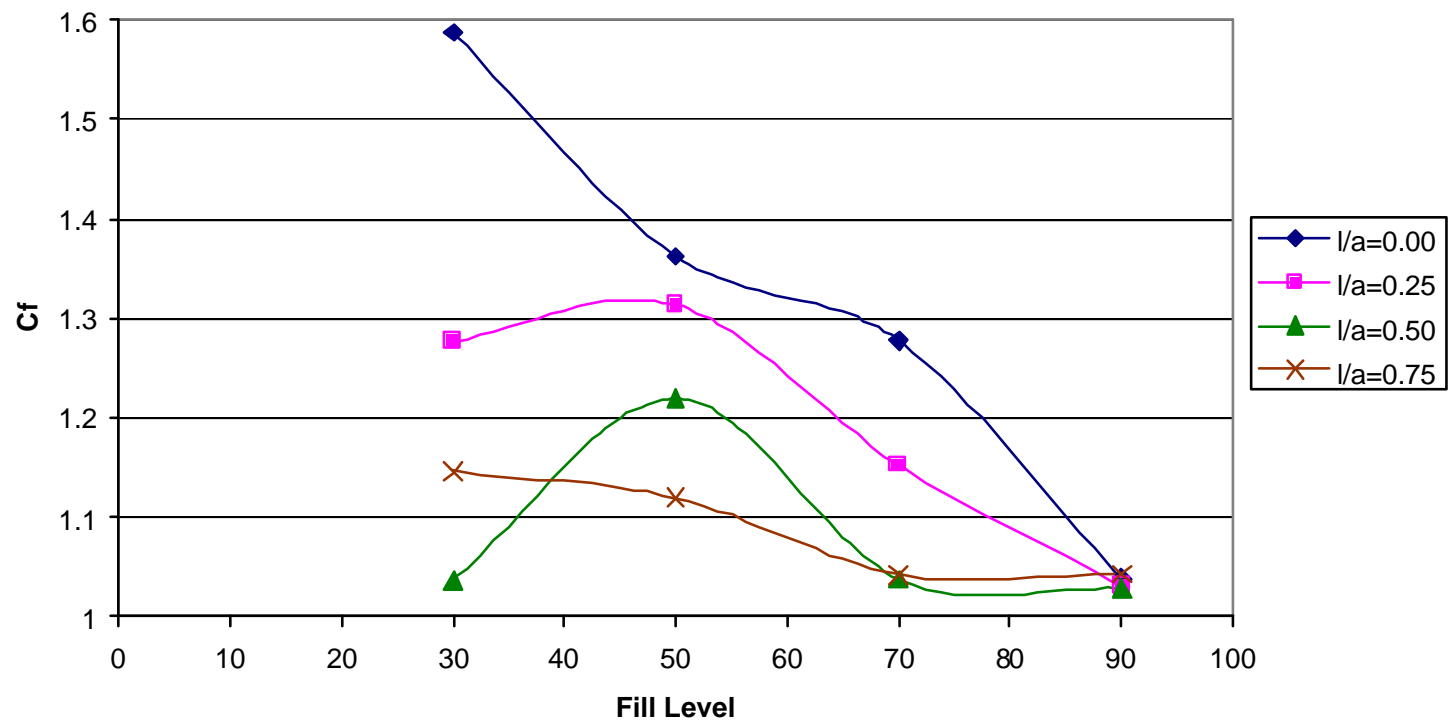

Figure $40 . \mathrm{C}_{\mathrm{f}}$ for circular tank with lateral acceleration of $0.3 \mathrm{~g}$ and diagonal baffles

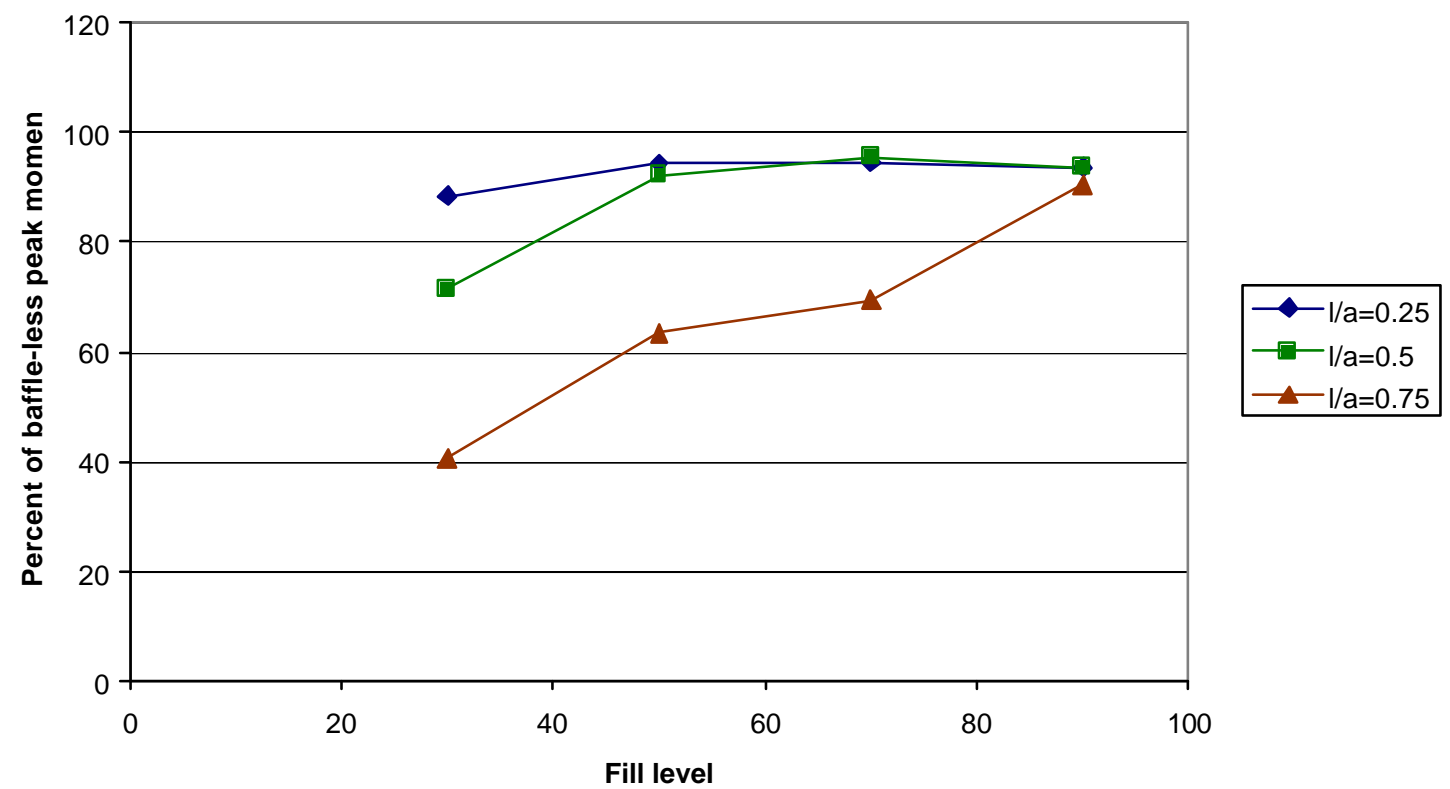

Figure 41. Percentage ratios of peak moments in a circular tank with horizontal-vertical baffles to peak moments in a circular tank without any baffles 


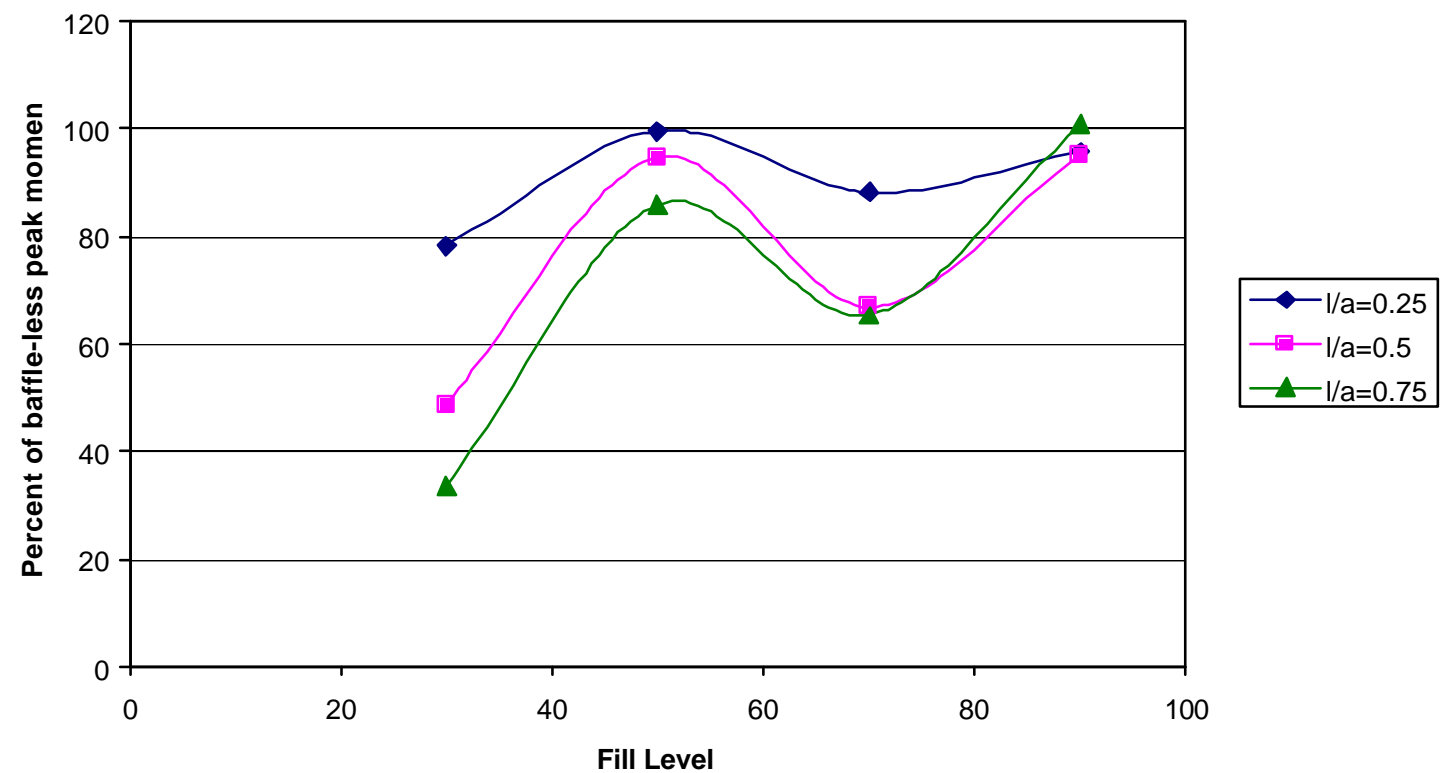

Figure 42. Percentage ratios of peak moments in a circular tank with diagonal baffles to peak moments in a circular tank without any baffles

In all the baffle designs that were considered, an 1/a ratio of 0.75 was more effective than other baffle lengths. The four configurations of baffles have different effects for different fill levels. The most suitable baffle lengths, in all the configurations were determined, and are shown in Figure 43. The best baffle configuration can be determined by comparing the reduction of peak moments provided by different configurations. Figure 44 shows the moment acting at the bottom of a circular ank with $70 \%$ fill level and going through a constant radius turn. It shows moments for all the configurations of baffles with an 1/a ratio of 0.75 . Comparison of moments in tanks with various baffles gives a fair understanding of the effects of various baffles on sloshing and moments. 


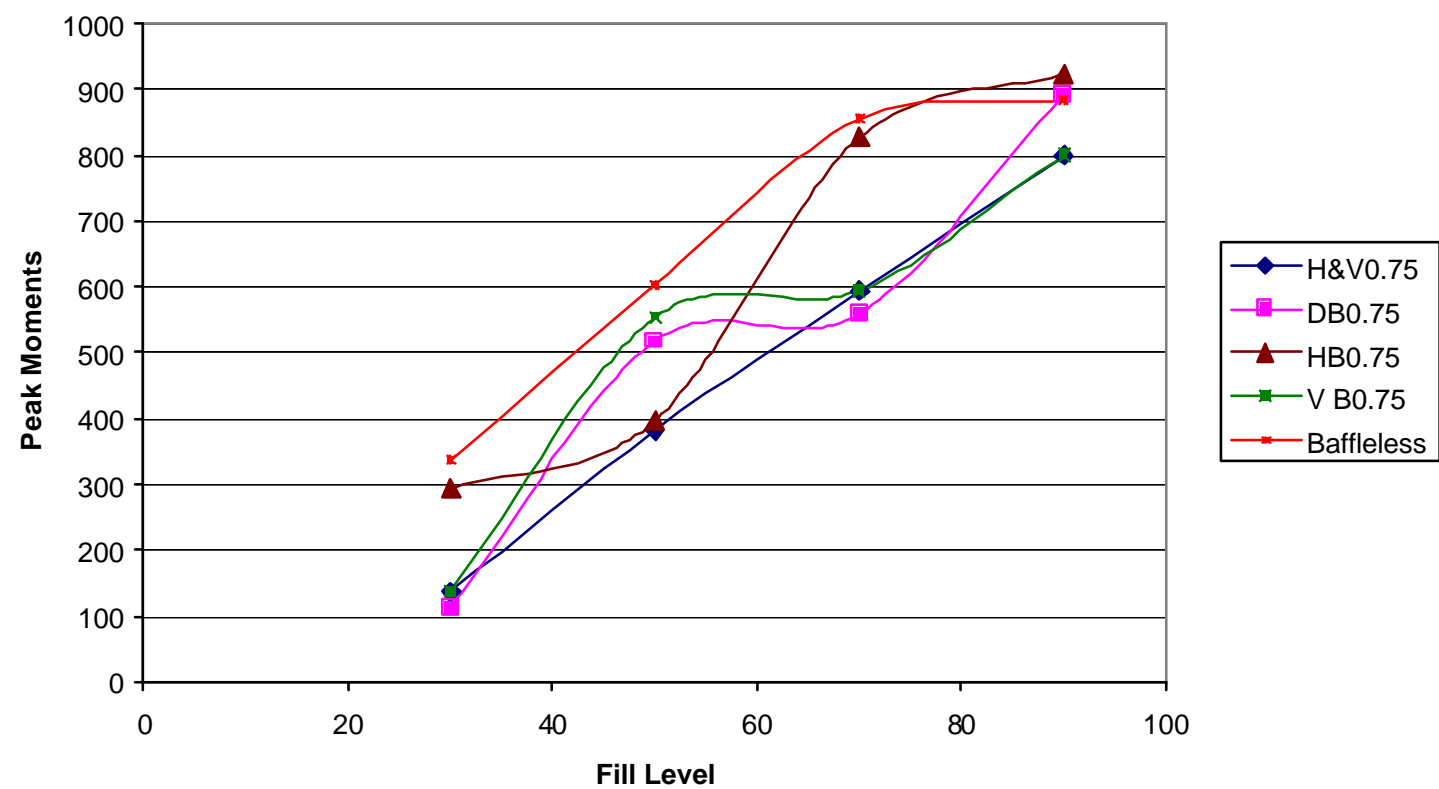

Figure 43. Peak moments for various baffles with $1 / \mathrm{a}=0.75$ in a circular tank through constant radius turn

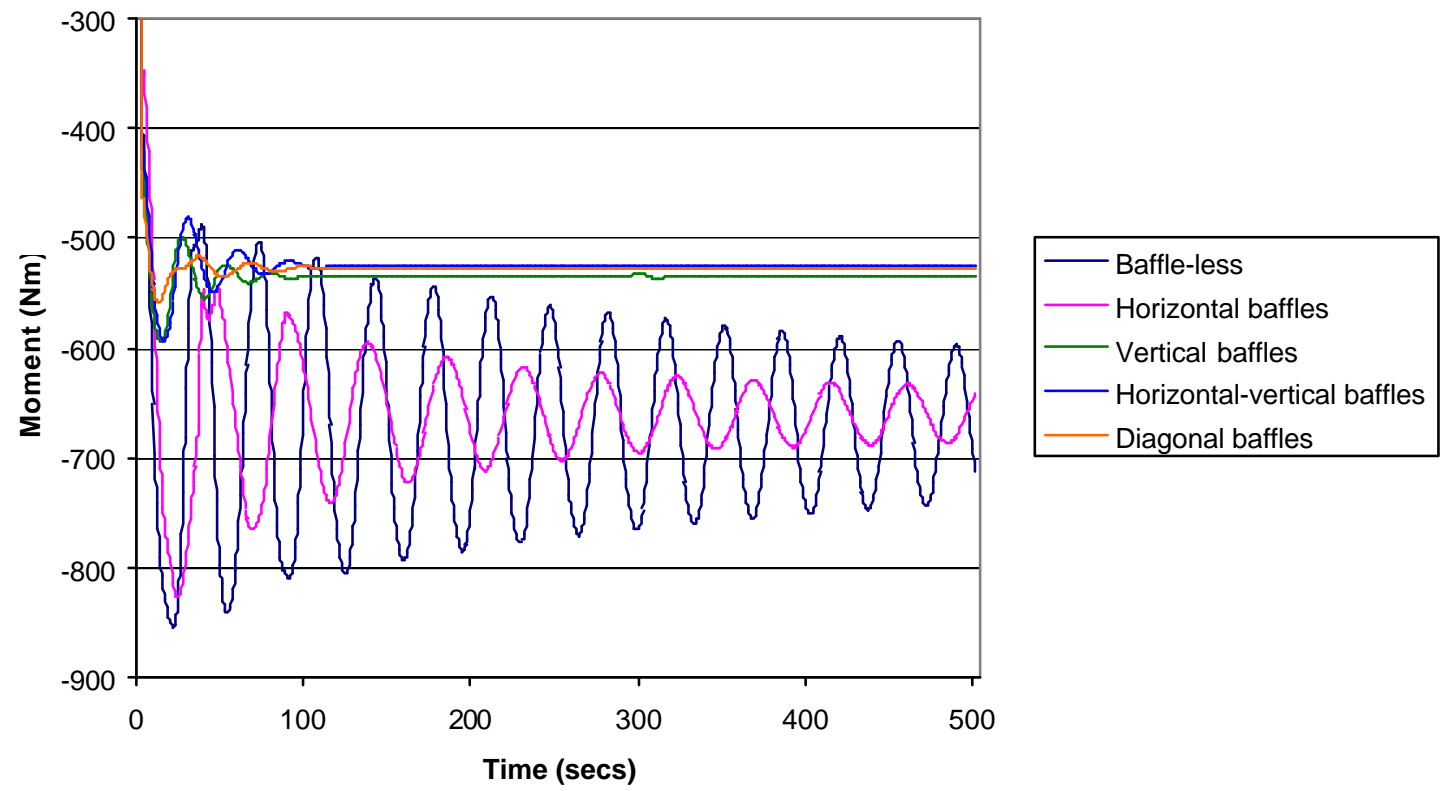

Figure 44. Moment with time for various baffles in a circular tank 
Figure 43 shows that diagonal baffles yield greater reduction for fill levels of $30 \%$ and $70 \%$. Vertical baffles provide more reduction in moment than horizontal baffles for all fill levels except $50 \%$. This can be attributed to the fact that damping is provided at the free surface of the fluid, in this case by horizontal baffles. At the other fill levels, horizontal baffles were either away form the free surface (30\% fill level) or immersed totally in the fluid (70\% and $90 \%$ fill levels). That makes them less effective for those fill levels. Vertical baffles were more effective for these fill levels. Thus, it was observed that the horizontal baffles and vertical baffles behave exactly in the opposite manner. Horizontal-vertical baffles have the added effect of both horizontal baffles and vertical baffles. That makes the configuration of horizontal-vertical baffles effective for all fill levels. Although, diagonal baffles give a greater reduction for $30 \%$ and $70 \%$ fill levels, the horizontal-vertical baffles was determined to be the best configuration for all the fill levels for a circular tank undergoing a constant radius turn.

Figure 44 also shows that horizontal-vertical baffles are the most effective baffles for $70 \%$ fill level. Horizontal baffles could not dampen the fluid oscillations effectively in this case due to their being immersed under the fluid. Vertical baffles, diagonal baffles, and horizontal-vertical baffles are all present at the fluid surface at $70 \%$ fill level and they could dampen the fluid oscillations significantly. This has been illustrated clearly in Figure 44.

New set of similar analyses was done with elliptical tank to determine the best configuration of baffles in an elliptical tank undergoing a constant radius turn. 


\subsubsection{ELLIPTICAL TANK}

An elliptical tank with an aspect ratio of 2 was considered in this study. Circular tank is essentially an elliptical tank with an aspect ratio of 1 . Similar set of analyses was run in FLUENT to determine the forces and moments on the elliptical tank walls. The forces and moments obtained from FLUENT were used to calculate the dynamic coefficients of force and moment for an elliptical tank going through a constant radius turn. All the four configurations of baffles, that is, horizontal baffles, vertical baffles, horizontal and vertical baffles, and diagonal baffles were considered again for this purpose. All results are shown in the following figures.

Figures 45, 46 and 47 show that horizontal baffles have the same effect in an elliptical tank as in a circular tank. The $1 / \mathrm{a}$ ratio of 0.75 yielded more reduction in peak moments up to a fill level of $70 \%$. For fill levels higher than $70 \%$, 1/a ratio of 0.5 appeared to be better, in reducing both peak moments and sloshing, than other lengths of baffles. As expected, the maximum reduction was observed for $50 \%$ fill level because of the damping at the free surface of the fluid. No significant reduction was observed for a 90\% fill level because the baffles were completely immersed inside the fluid and did not seem to have an effect. The maximum reduction obtained in this case was $38 \%$, which is 4\% higher than that obtained in a circular tank with horizontal baffles. Percent reductions obtained in peak moments at $30 \%$ and $70 \%$ fill levels were $31 \%$ and $19 \%$, respectively. No reduction was obtained at a $90 \%$ fill level. Figure 48 gives the percentage ratios of peak moments for all the baffle lengths in this case of an elliptical tank to the peak moments in a tank without any baffles. 


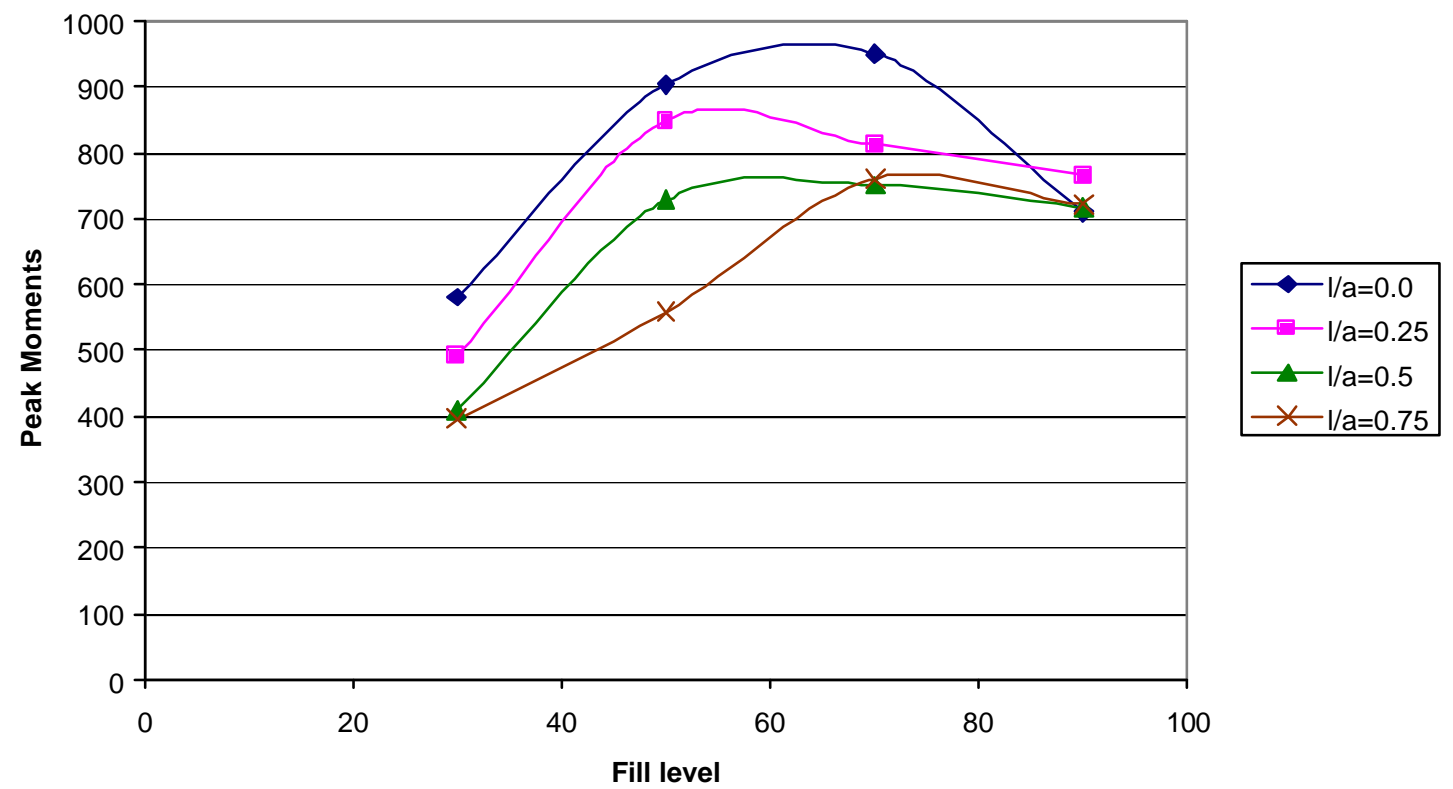

Figure 45. Peak moments for elliptical tank with lateral acceleration of $0.3 \mathrm{~g}$ and horizontal baffles

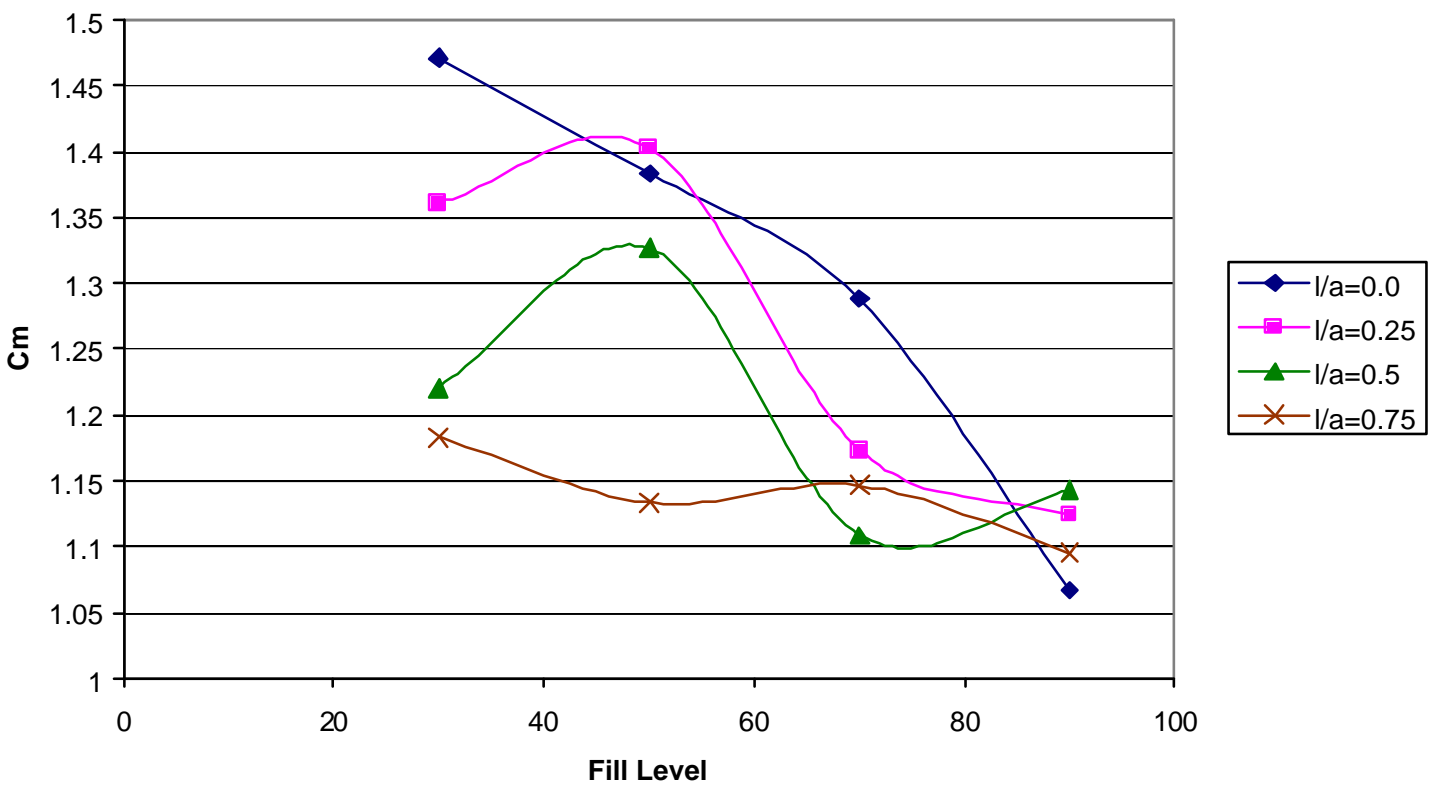

Figure $46 . \mathrm{C}_{\mathrm{m}}$ for elliptical tank with lateral acceleration of $0.3 \mathrm{~g}$ and horizontal baffles 


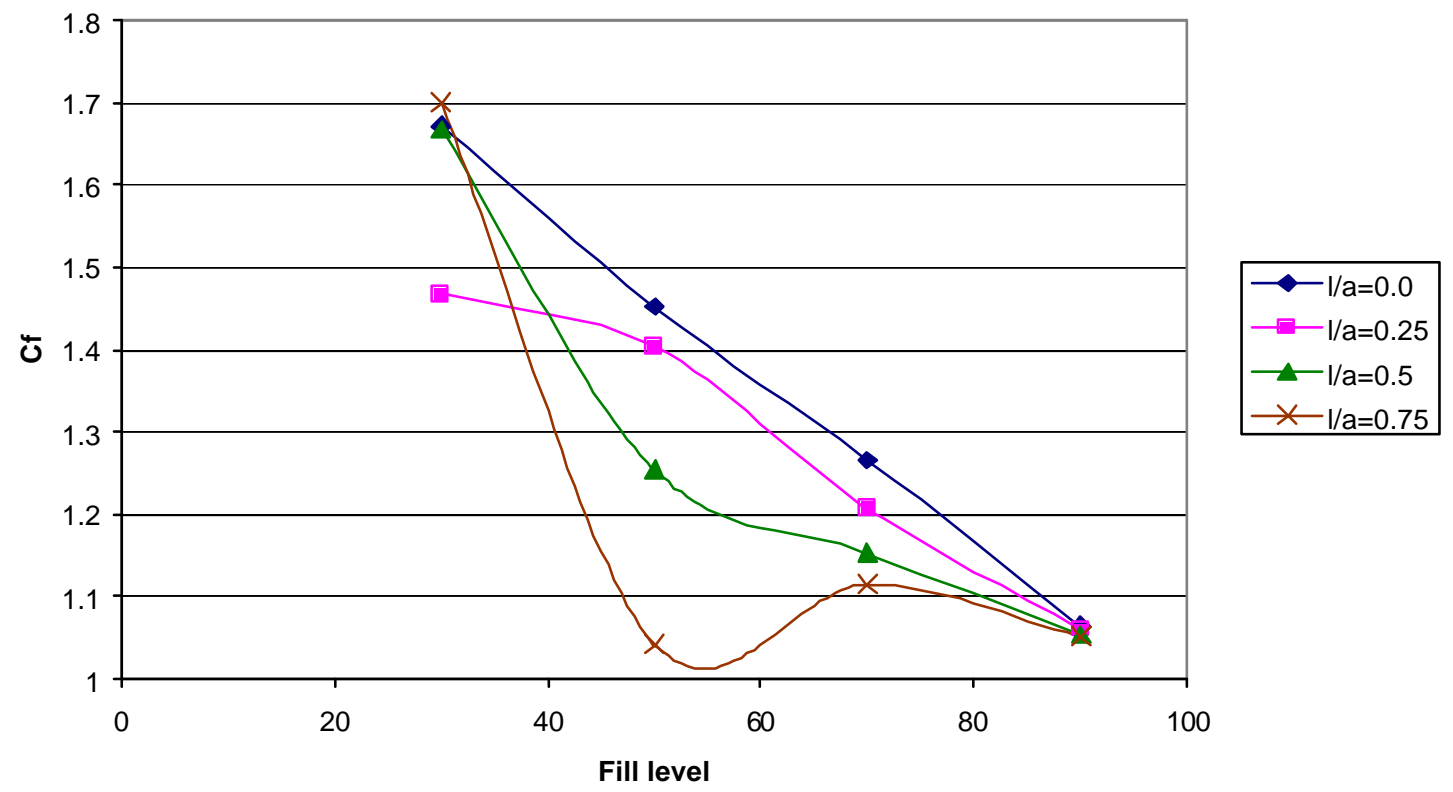

Figure 47. $\mathrm{C}_{\mathrm{f}}$ for circular tank with lateral acceleration of $0.3 \mathrm{~g}$ and horizontal baffles

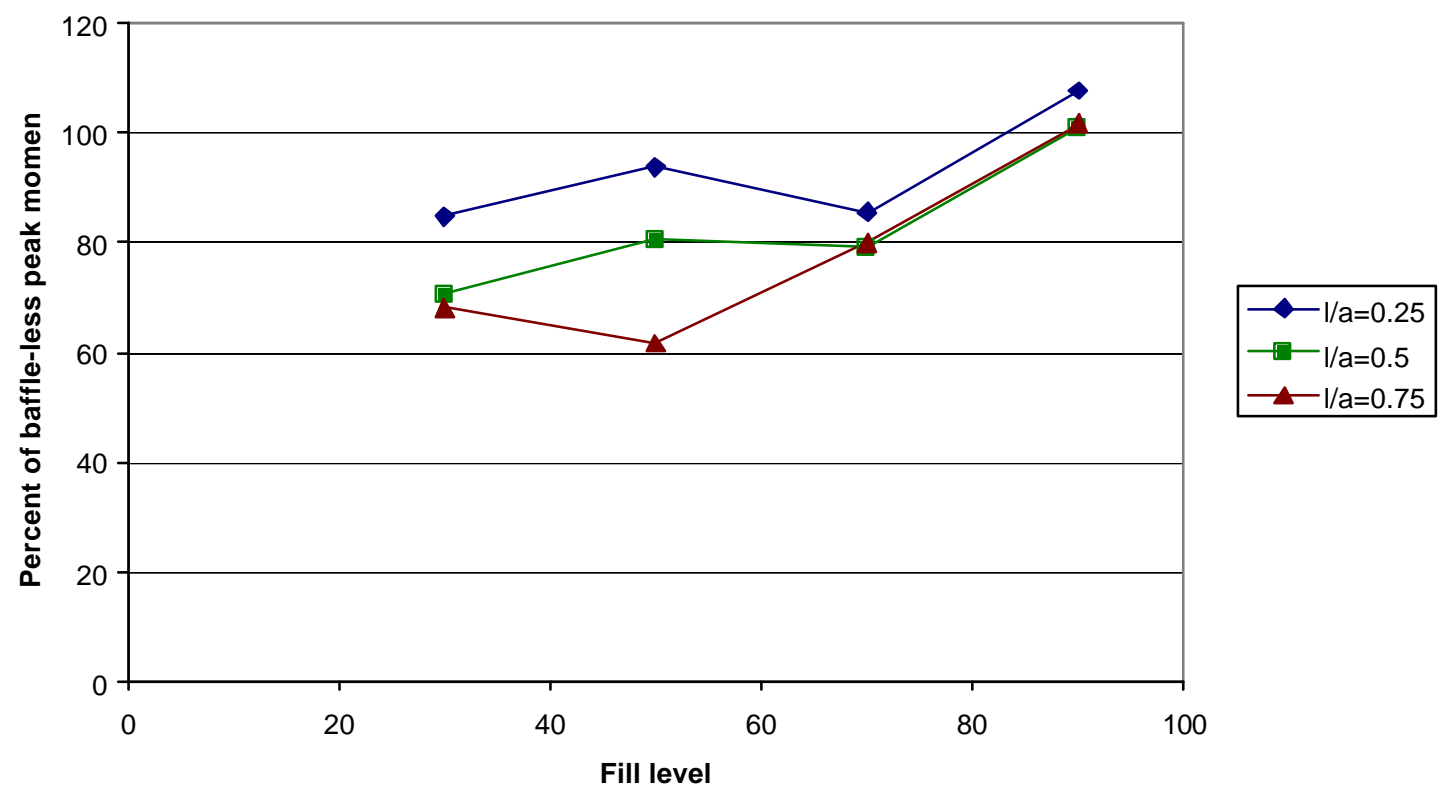

Figure 48. Percentage ratios of peak moments in an elliptical tank with horizontal baffles to peak moments without any baffles 


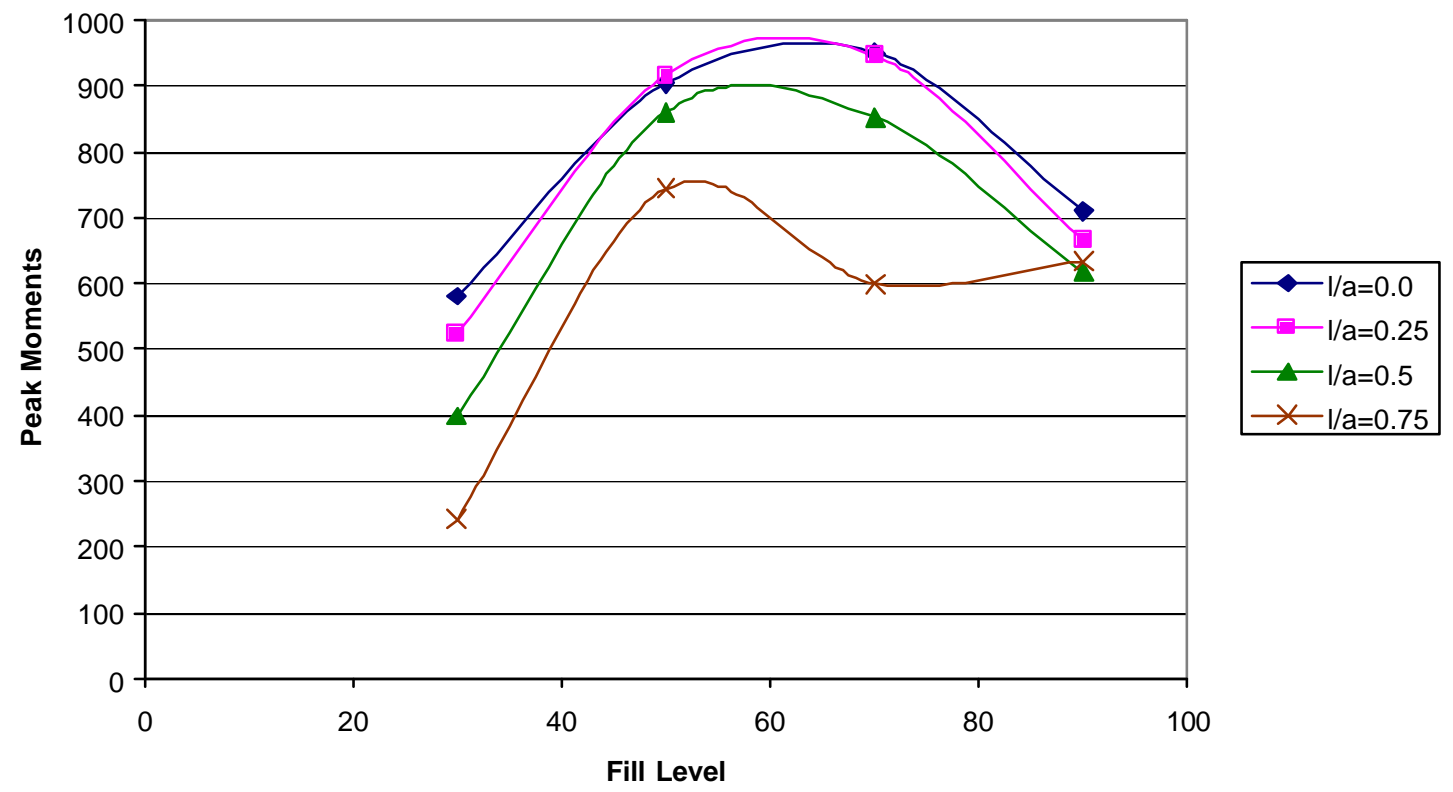

Figure 49. Peak moments for elliptical tank with lateral acceleration of $0.3 \mathrm{~g}$ and vertical baffles

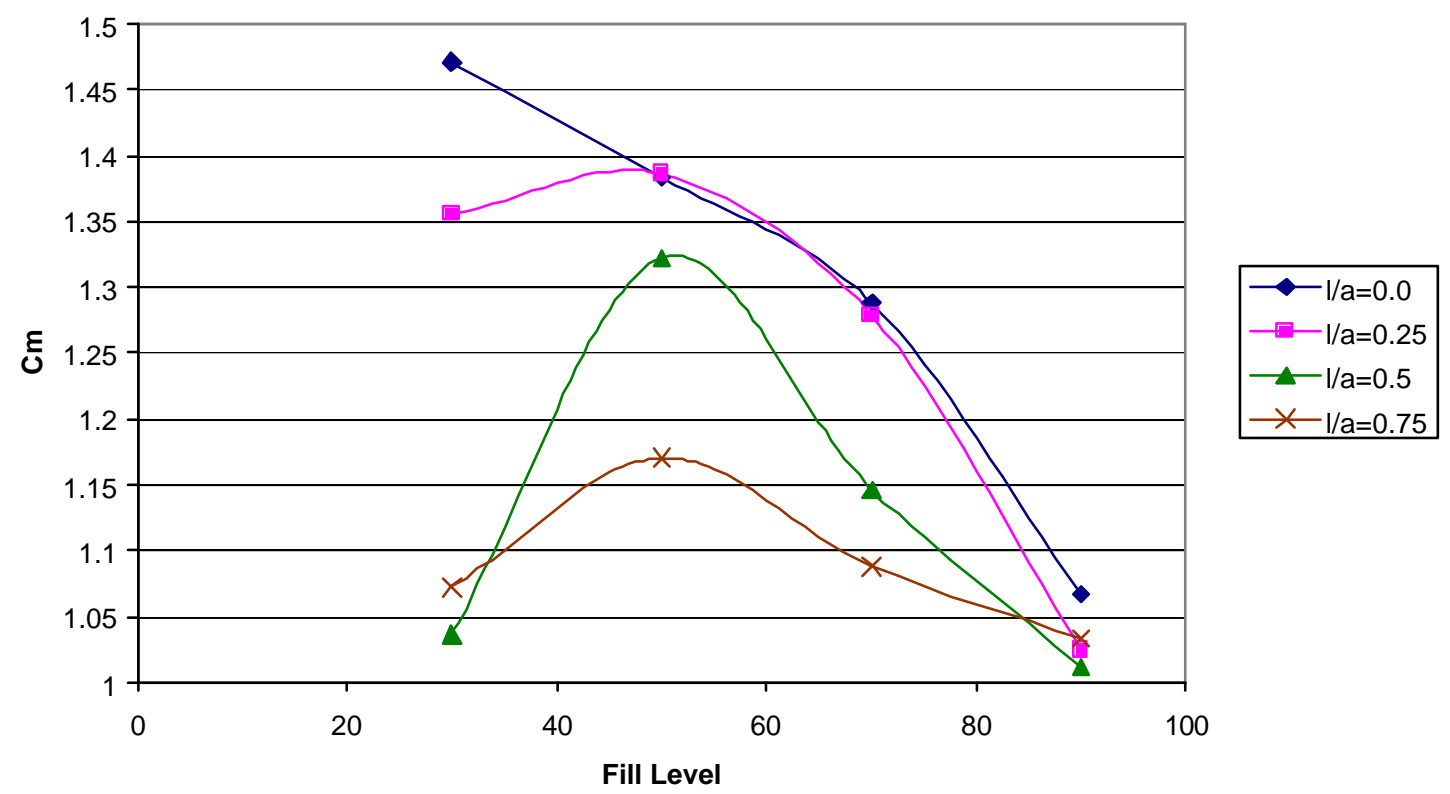

Figure $50 . \mathrm{C}_{\mathrm{m}}$ for elliptical tank with lateral acceleration of $0.3 \mathrm{~g}$ and vertical baffles 


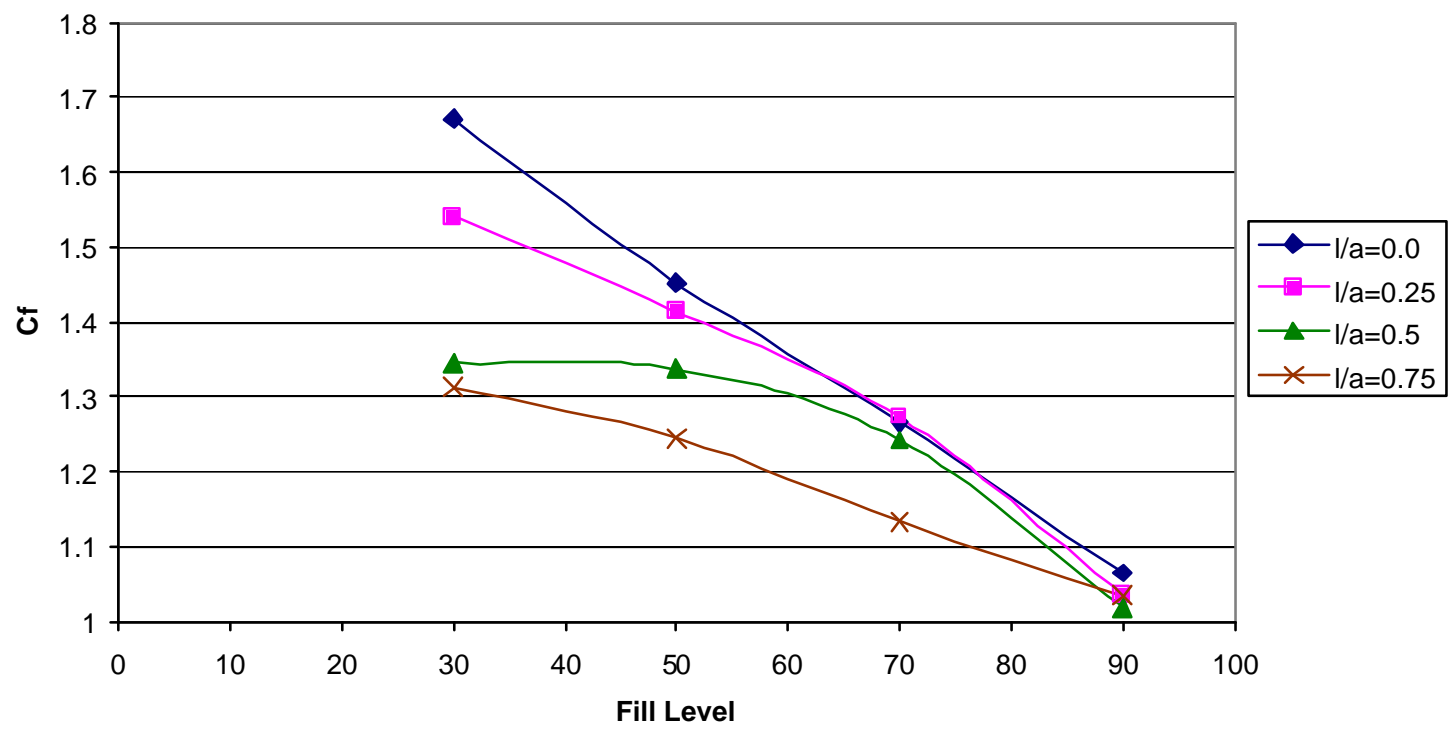

Figure $51 . \mathrm{C}_{\mathrm{f}}$ for elliptical tank with lateral acceleration of $0.3 \mathrm{~g}$ and vertical baffles

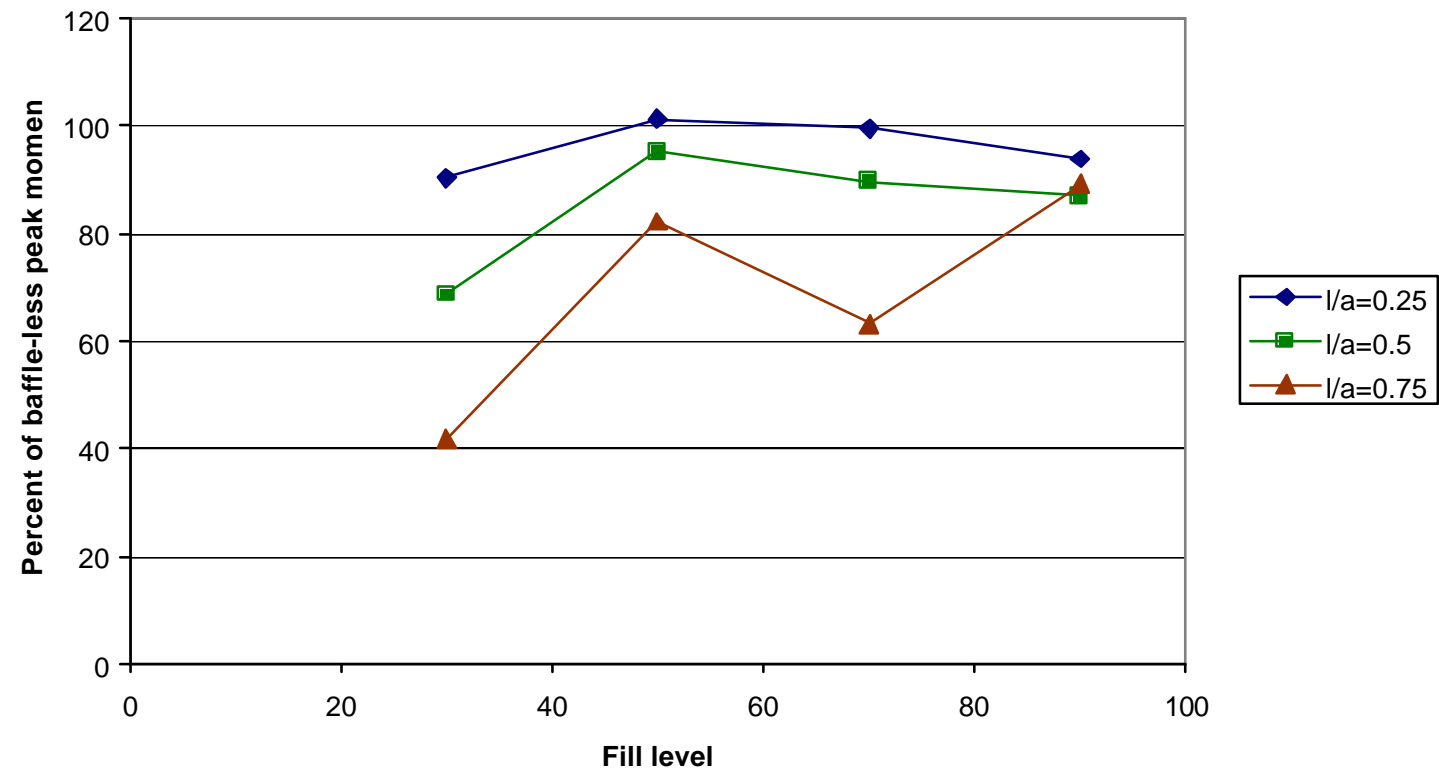

Figure 52. Percentage ratios of peak moments in an elliptical tank with vertical baffles to peak moments without any baffles 
Figures 49, 50 and 51 show that vertical baffles in an elliptical tank maneuvering a constant radius turn had the same effect as the vertical baffles in a circular tank maneuvering a constant radius turn. An $1 / \mathrm{a}$ ratio of 0.75 was found to be more effective than other lengths of the baffles. A maximum reduction of $58 \%$ was observed for $30 \%$ fill level with these baffles. A reduction of $18 \%$ was obtained for a fill of $50 \%$. Fill level of $70 \%$ resulted in a reduction of $36 \%$ with these baffles. Figure 52 gives the percentage ratios of peak moments for all the baffle lengths (vertical baffles) in this case of an elliptical tank to the peak moments in a tank without any baffles. Horizontal-vertical baffles were considered next.

It is evident from Figures 53, 54 and 55 that horizontal-vertical baffles had a more positive effect than the horizontal baffles or vertical baffles in an elliptical tank going through a constant radius turn. An 1/a ratio of 0.75 gave the most reduction in both peak moments and dynamic coefficients compared to other $1 /$ a ratios of the baffles. A reduction of $62 \%$ in the peak moment was observed for a $30 \%$ fill level. Reductions of $36 \%$ and $42 \%$ were observed for fill levels $50 \%$ and $70 \%$ respectively for an elliptical tank with horizontal-vertical baffles maneuvering a constant radius turn. Figure 56 gives the percentage ratios of peak moments for all the baffle lengths (horizontal-vertical baffles) in this case of an elliptical tank to the peak moments in a tank without any baffles. Diagonal baffles were considered next. 


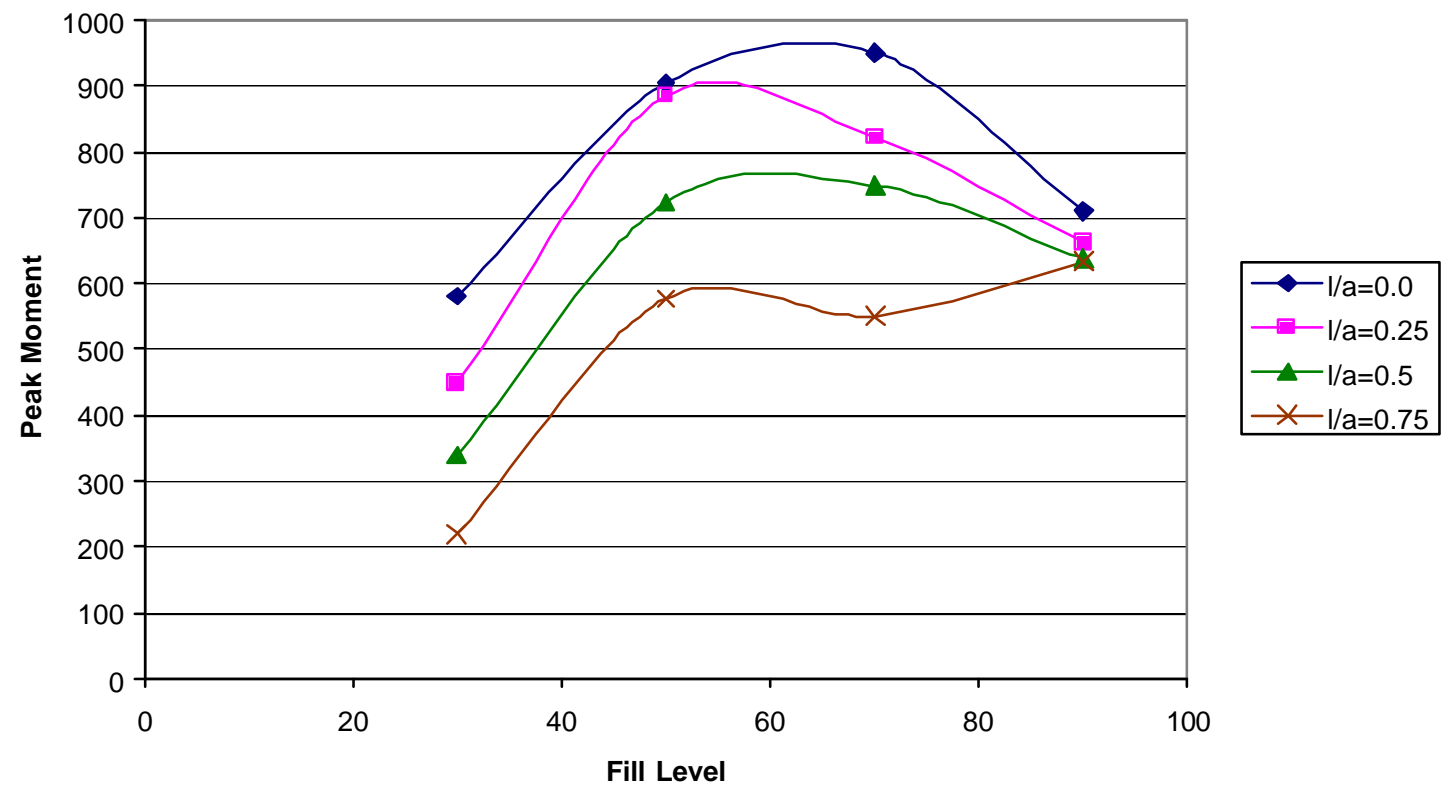

Figure 53. Peak moments for elliptical tank with lateral acceleration of $0.3 \mathrm{~g}$ and horizontal-vertical baffles

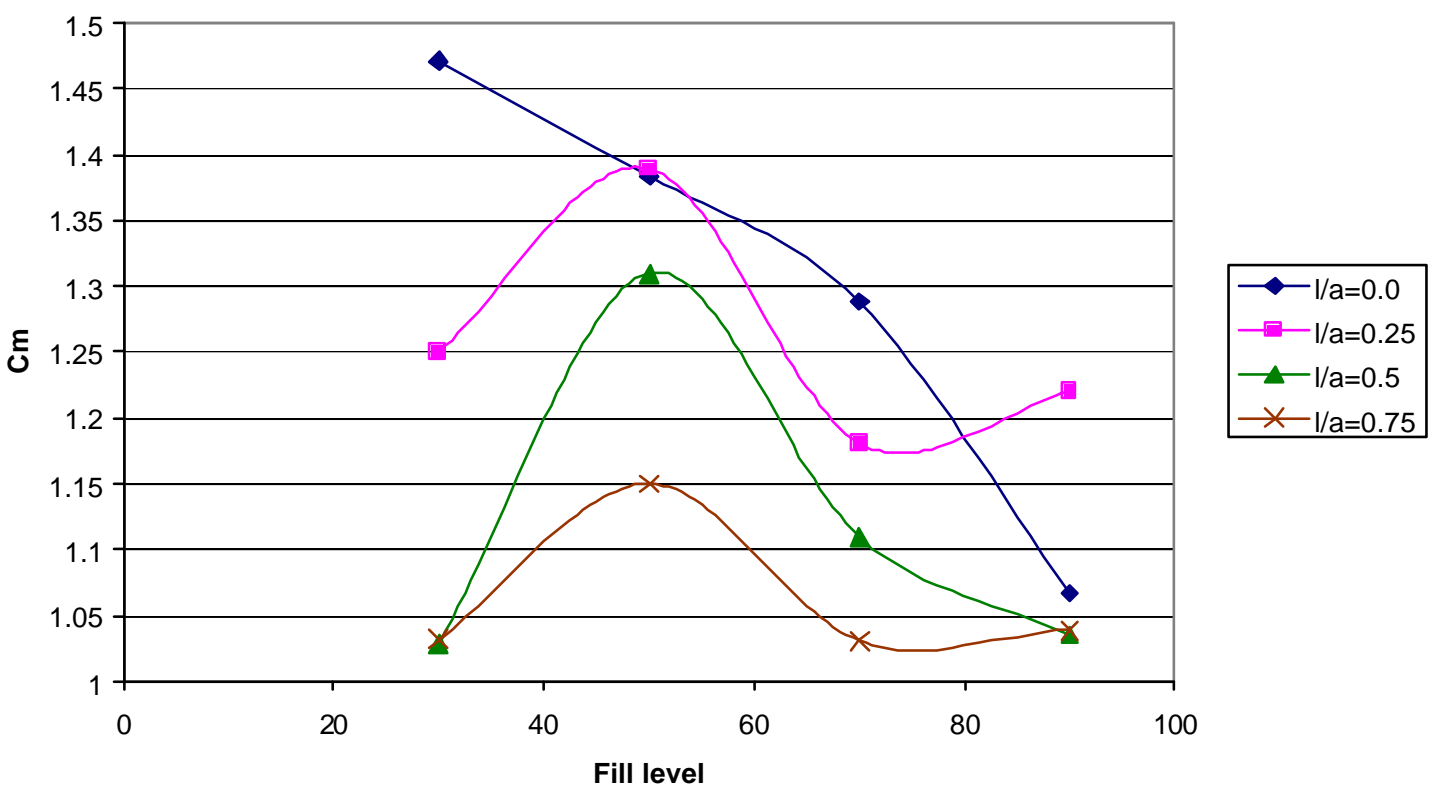

Figure $54 . C_{m}$ for elliptical tank with lateral acceleration of $0.3 \mathrm{~g}$ and horizontal-vertical baffles 


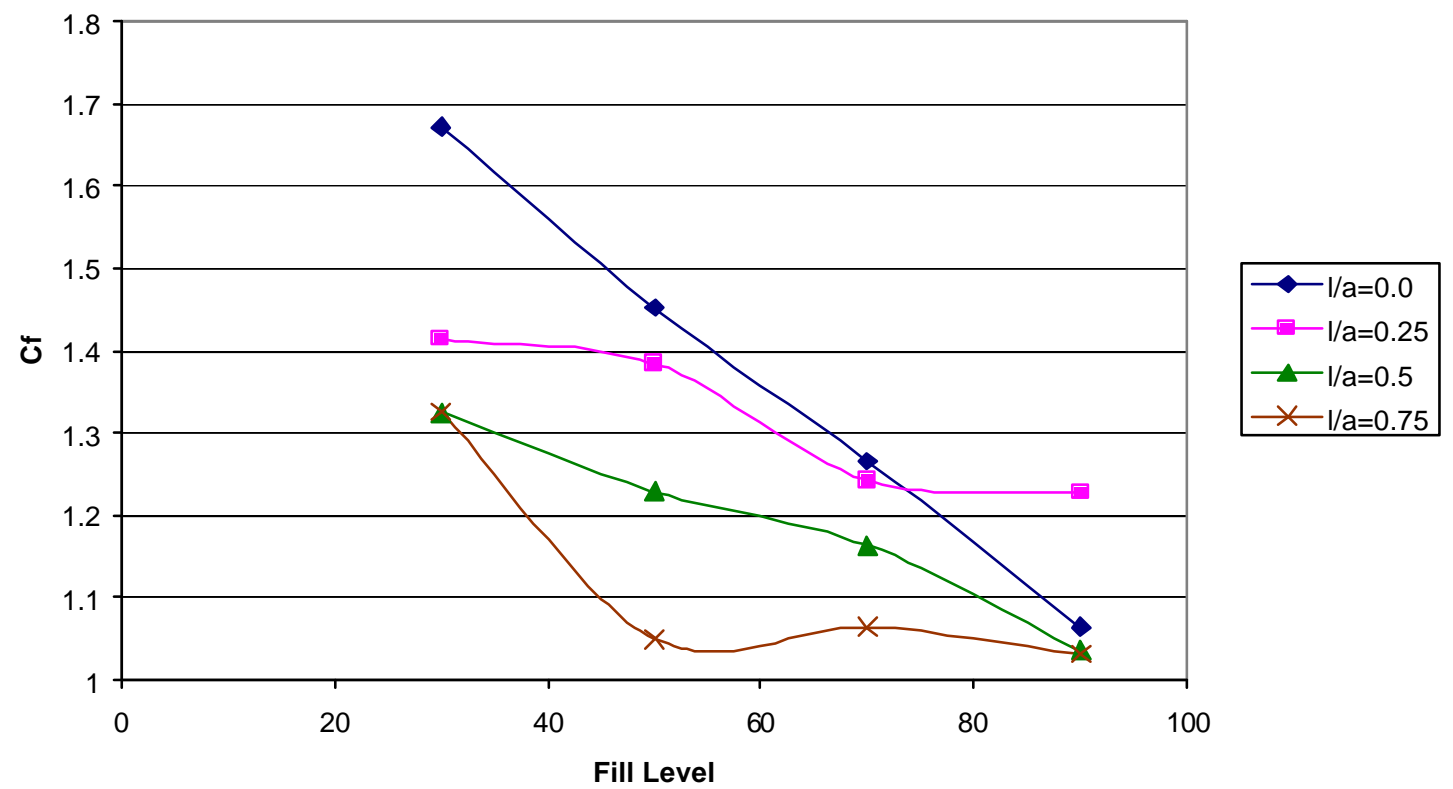

Figure $55 . \mathrm{C}_{\mathrm{f}}$ for elliptical tank with lateral acceleration of $0.3 \mathrm{~g}$ and horizontal-vertical baffles

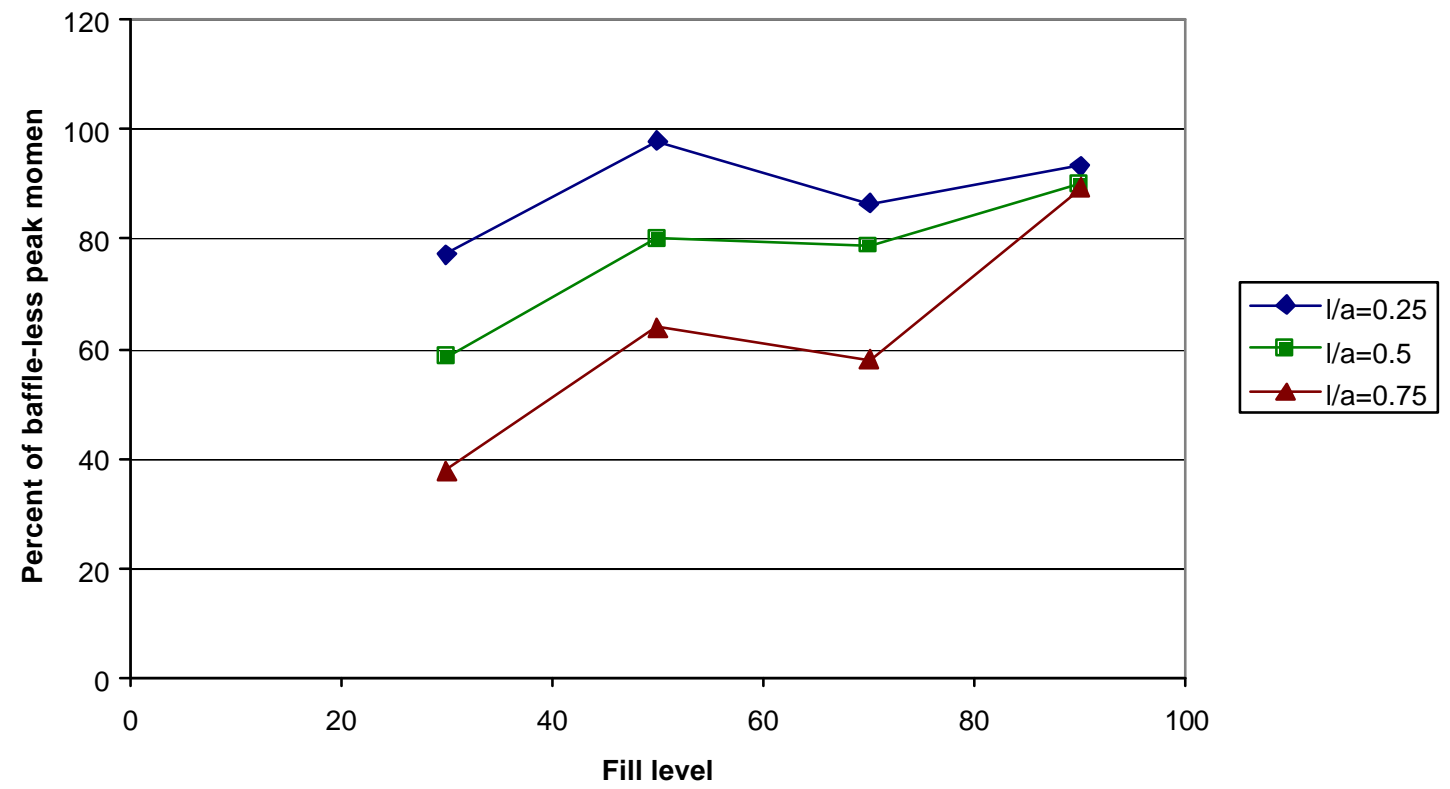

Figure 56. Percentage ratios of peak moments in an elliptical tank with horizontal-vertical baffles to peak moments without any baffles 


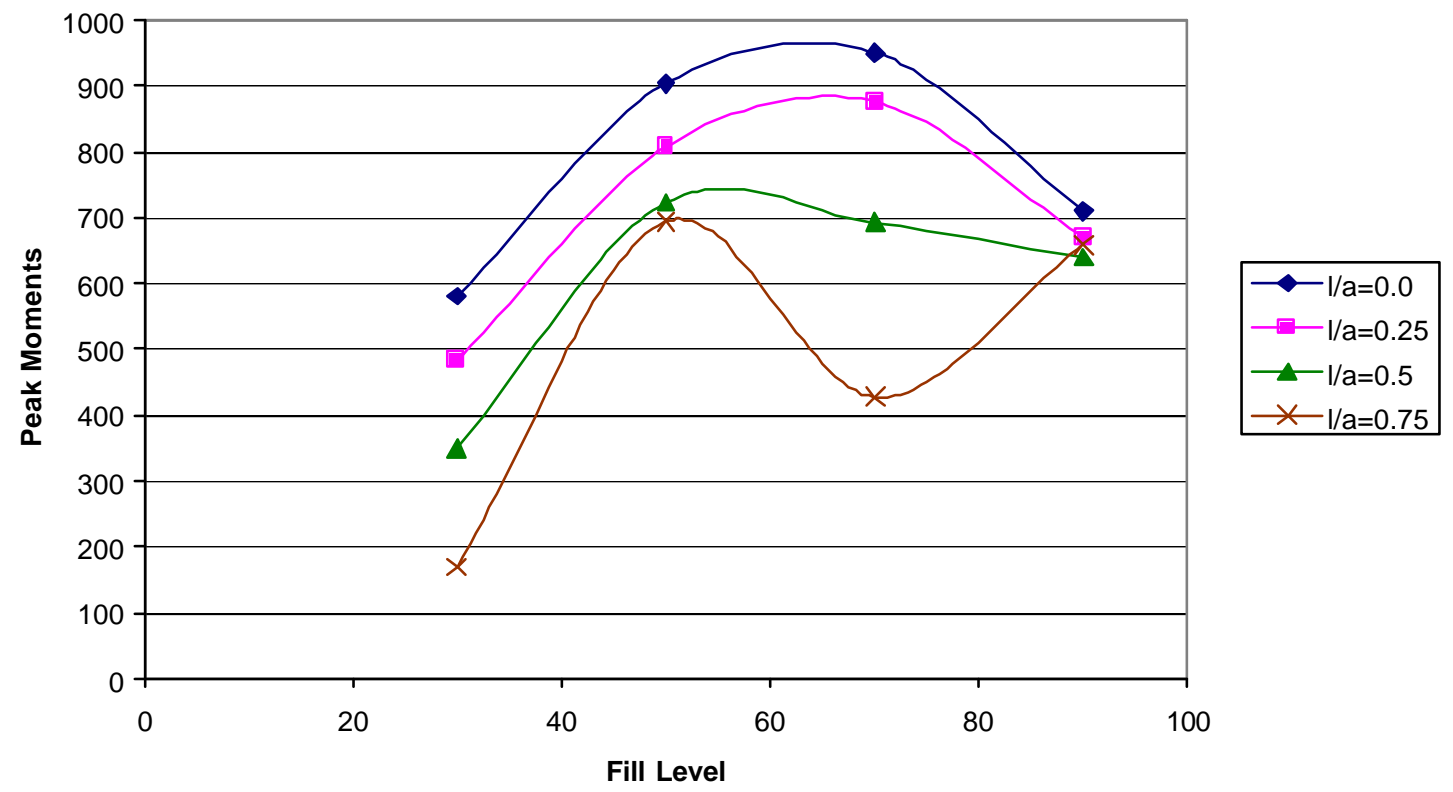

Figure 57. Peak moments for elliptical tank with lateral acceleration of $0.3 \mathrm{~g}$ and diagonal baffles

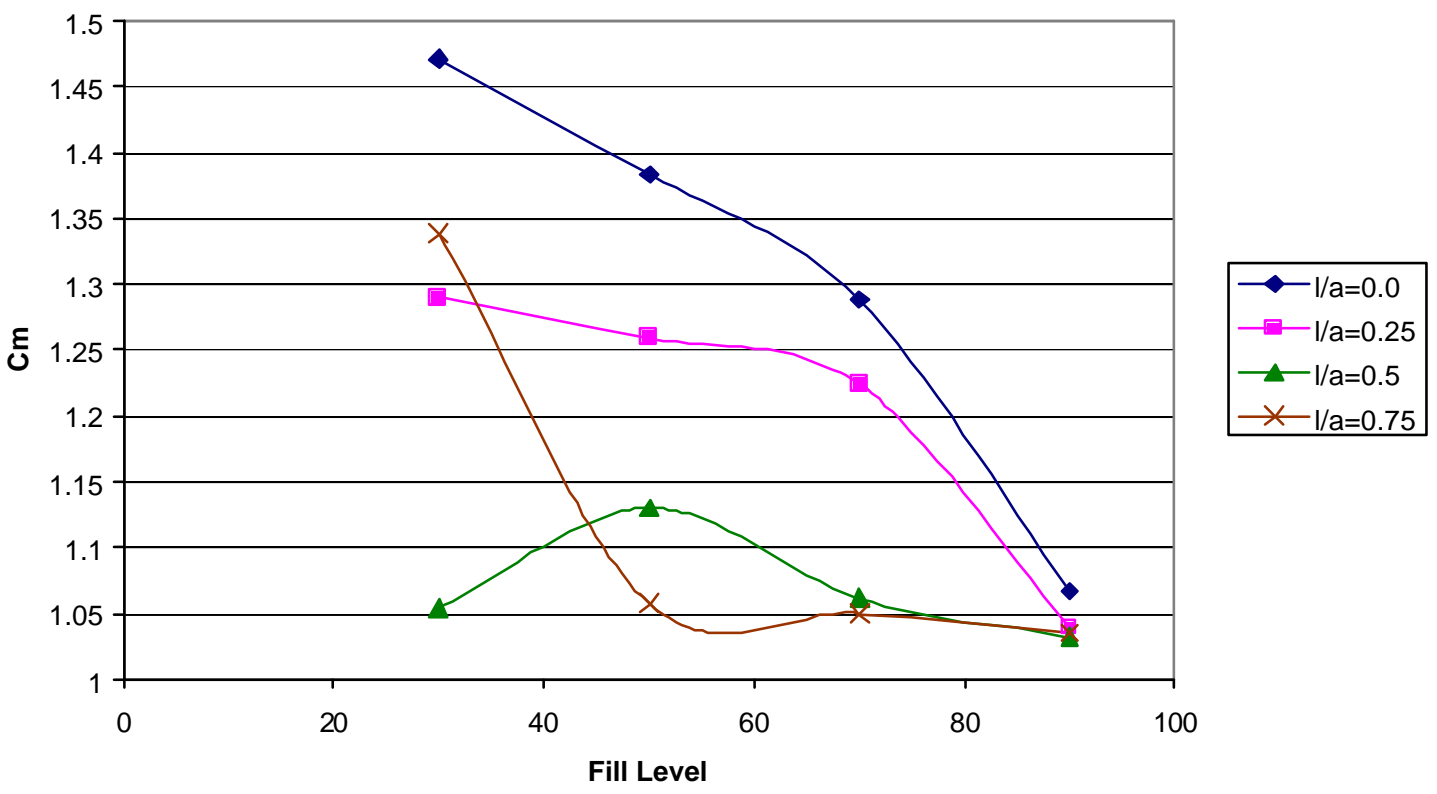

Figure $58 . \mathrm{C}_{\mathrm{m}}$ for elliptical tank with lateral acceleration of $0.3 \mathrm{~g}$ and diagonal baffles 


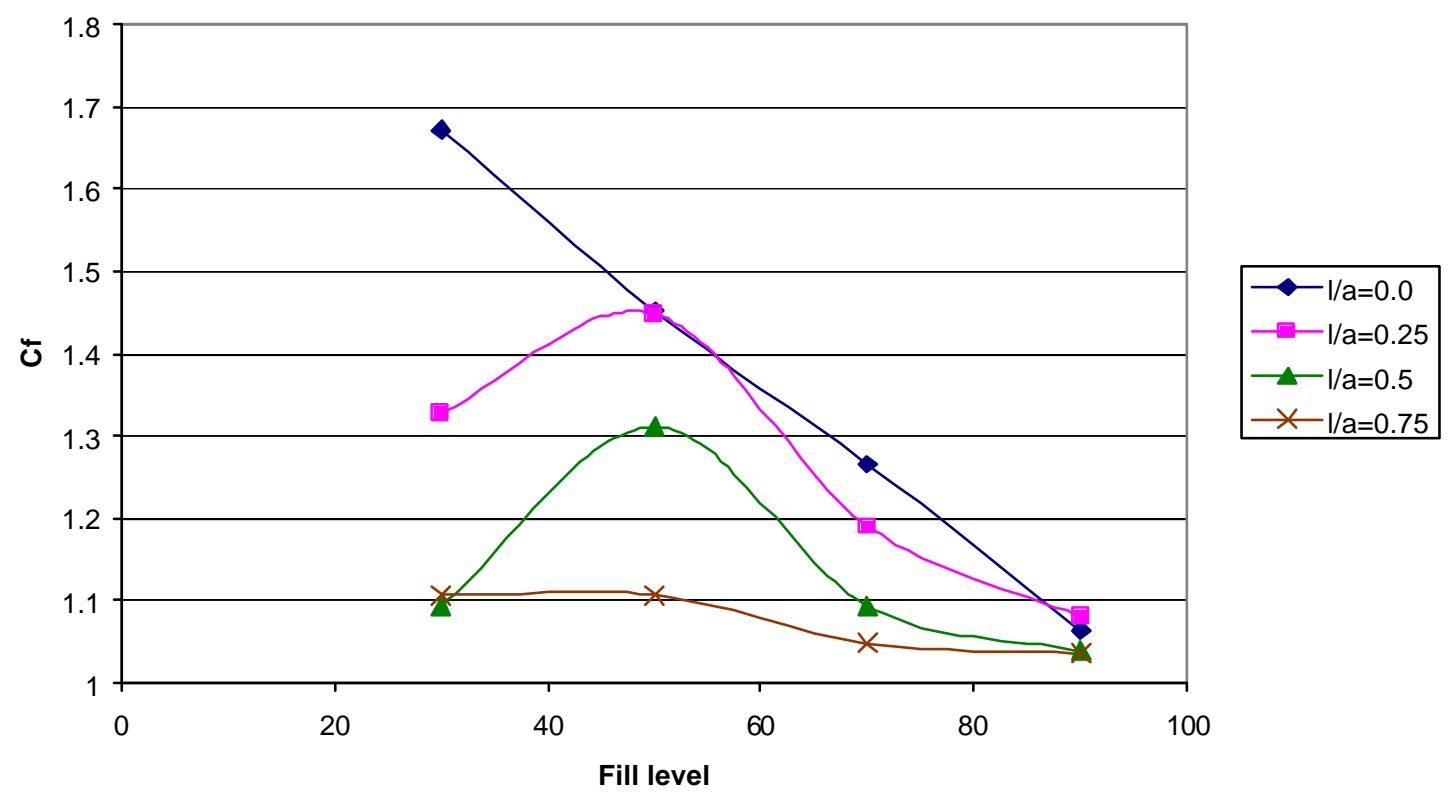

Figure $59 . \mathrm{C}_{\mathrm{f}}$ for elliptical tank with lateral acceleration of $0.3 \mathrm{~g}$ and diagonal baffles

Similar to the effect in circular tank diagonal baffles with an 1/a ratio of 0.75 were found to be more effective, as shown in Figures 57, 58 and 59, in reducing both the moments and sloshing for all fill levels except for fill 30 where 1/a ratio of 0.5 had a greater reduction in sloshing. But, moments at a fill level of $30 \%$ were reduced more by baffles with an $1 / \mathrm{a}$ ratio of 0.75 . Large reductions of $71 \%$ and $55 \%$ in the peak moment were obtained for fill levels of $30 \%$ and $70 \%$, respectively. These are the fill levels where diagonal baffles have the best effect. Reductions of $24 \%$ and $7 \%$ were obtained for fill levels of $50 \%$ and $90 \%$, respectively. For these two fill levels horizontal-vertical baffles were determined to be the best configuration. This is shown in Figure 60 where the effects of optimized baffle lengths in all configurations are plotted together. 


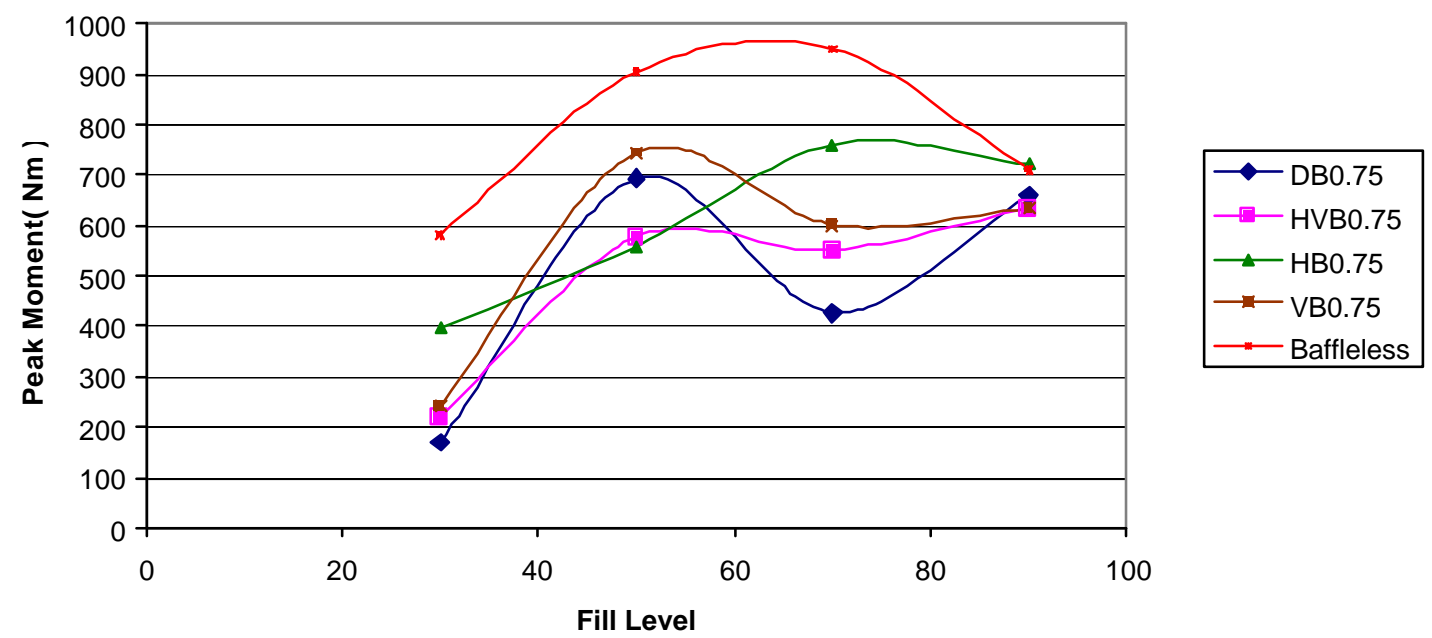

Figure 60. Peak moments for various baffles with $1 / \mathrm{a}=0.75$ in an elliptical tank through

constant radius turn

As found in the case of circular tank diagonal baffles were found to be the best configuration for elliptical tank at fill levels of $30 \%$ and $70 \%$. Horizontal-vertical baffles were the best choice for other fill levels. Figures showing the percentage ratios of peak moments for all the baffle lengths (diagonal baffles) in this case of an elliptical tank to the peak moments in a tank without any baffles, and moments at the bottom of the tank with various baffles are shown in the Appendix A.

Effects of various baffle configurations on reducing moments and sloshing in the case of a TOP lane change maneuver are described in the following sections.

\subsection{TOP LANE CHANGE MANEUVER}

Double lane change maneuver is another critical maneuver in the transportation of heavy-duty tanker trucks. TOP lane change and NATO lane change maneuvers are the 
two kinds of double lane change maneuvers that were investigated in the study conducted at Aberdeen Test Center (ATC). Figures 61 and 62 show the path of a vehicle in both these lane change maneuvers.

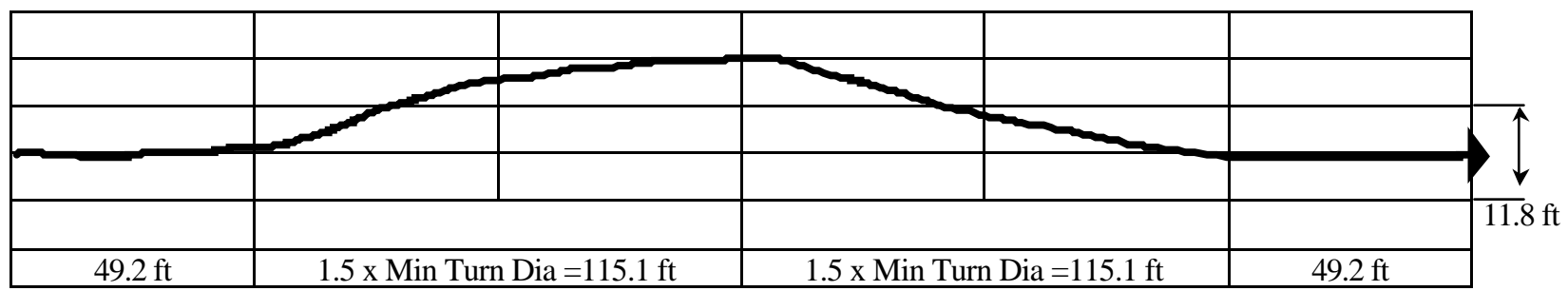

Figure 61. Path of the test vehicle during the TOP Lane Change Test

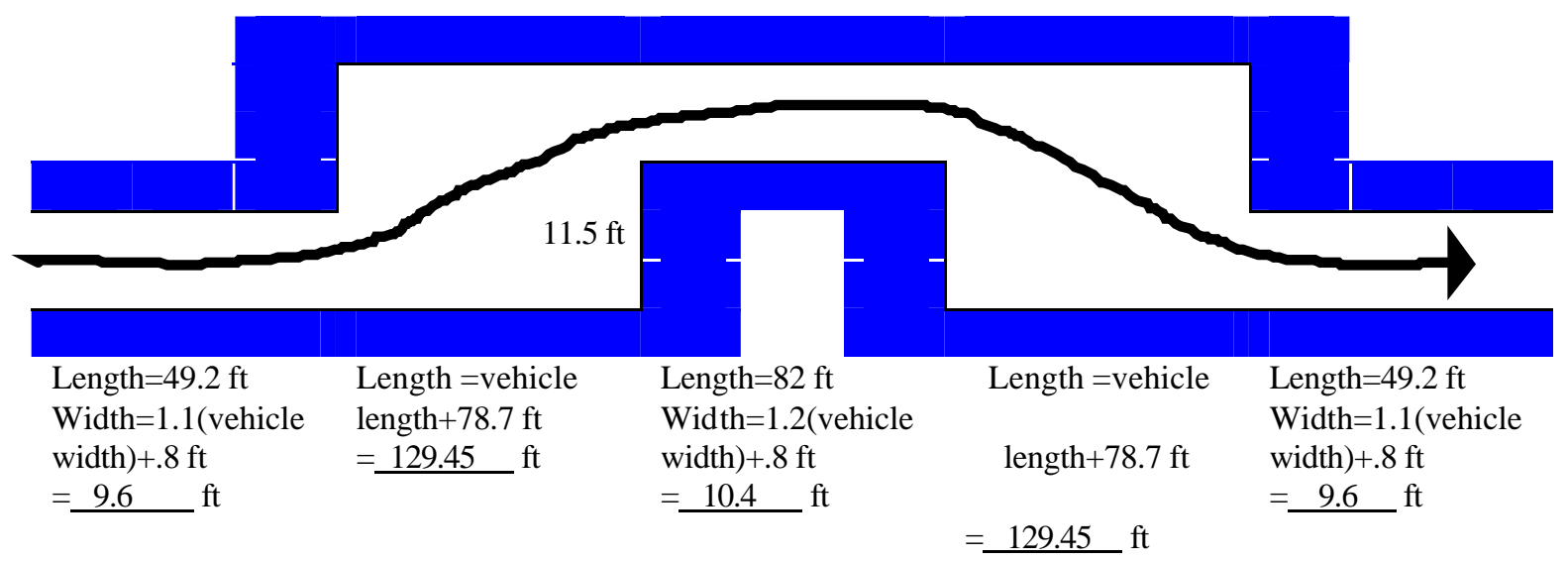

Figure 62. Path of the test vehicle during the NATO Lane Change Test

TOP lane change path was considered, in this study, to determine the effects of baffles in a double lane change maneuver. Determining the effect of various baffles in a double lane change maneuver is very important to draw any conclusions about the effects of baffles in tankers. A constant radius turn was simulated using a constant lateral acceleration that was equal to the centripetal acceleration acting on the fluid inside the 
tank. TOP lane change maneuver was simulated using the lateral acceleration calculated from the data provided by ATC.

Data from ATC contained the displacements of both tractor and tanker in X and $\mathrm{Y}$ directions with time. It also contained reactions on different wheels, and yaw and pitch angles of tractor and trailer. Most of the data from ATC required low band filtering to get rid of the excessive noise. The data was filtered using a MATLAB program written by Mohamed [18].

The lateral acceleration of the trailer was calculated by numerically differentiating the Y-coordinate of the trailer twice with respect to time using central difference method, as shown by the following equation:

$$
V_{n}=\frac{X_{n+1}-X_{n-1}}{2 \Delta t}
$$

The lateral acceleration thus obtained could be used to simulate the double lane change maneuver. Figure 63 shows the calculated lateral acceleration fr ATC TOP lane change maneuver for a tanker with a 50\% fill level.

The lateral acceleration calculation for the trailer shown in Figure 63 consisted of 833 time steps with each time step being 0.01 sec. Here, the acceleration changed with time unlike the case of a constant radius turn where the acceleration was constant throughout the maneuver. 


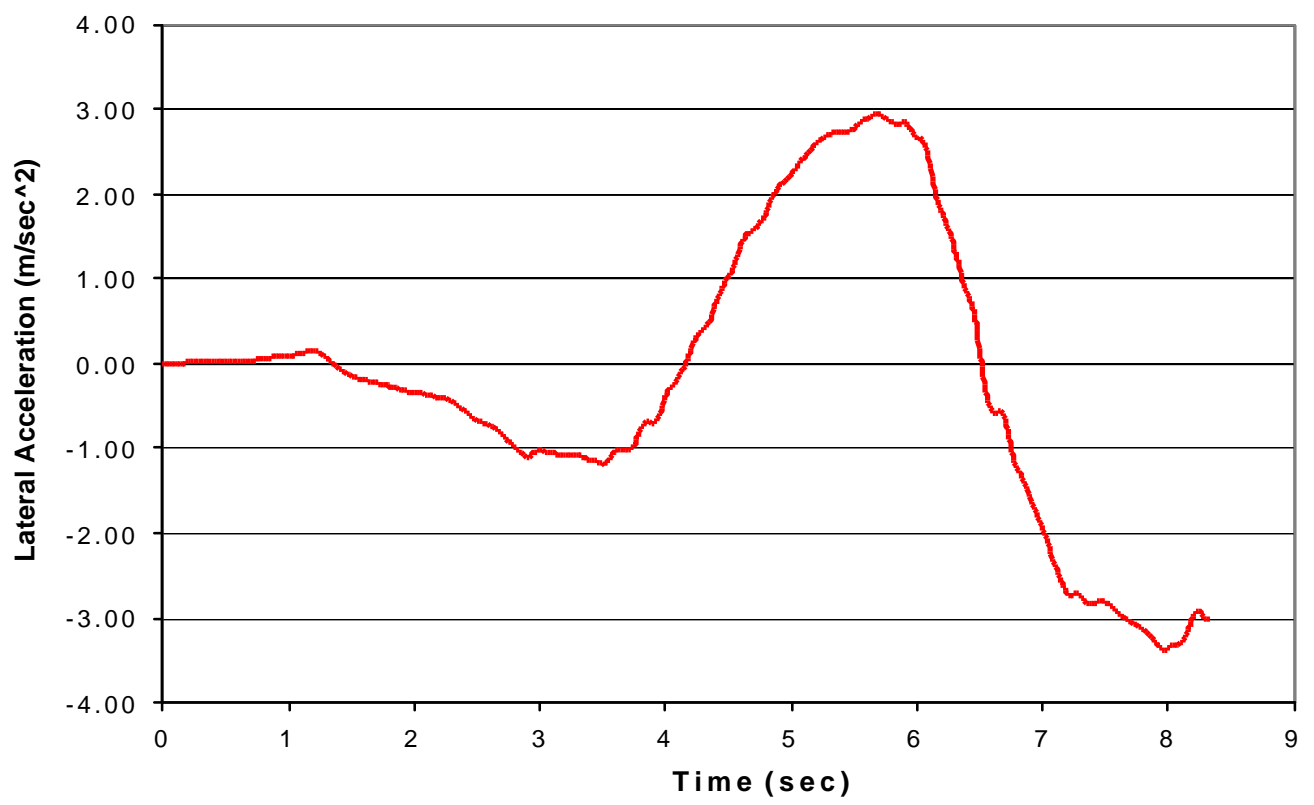

Figure 63. Calculated lateral acceleration for the trailer under study, at $50 \%$ full condition, during the TOP lane change test

The case of a constant acceleration was solved in FLUENT by setting the desired acceleration in the OPERATING SYSTEMS panel. After every time step FLUENT automatically checks for the acceleration in the operating conditions panel and goes from there. FLUENT does not have a provision to input a time-varying acceleration required in the TOP lane change maneuver.

A user defined sub routine, shown in the Appendix B, was written to apply the time-varying acceleration in the FLUENT. The program reads all the values of lateral acceleration with time from the "acceleration file" and returns the desired acceleration at any particular time as required by FLUENT. The user defined functions (UDF) update interval was set to 1 so that this program was called after every time step, and the acceleration was updated. Thus, the TOP lane change maneuver was simulated by applying the appropriate accelerations at different times during the analysis. 
The model developed with above modifications required several weeks of run time before it would converge. The user-defined function being called after every timestep, 833 discrete points in the acceleration file and the calculations involved after that were complicating the model and required a large amount of time to converge. A total of 96 tests had to be run for two different tank shapes with four configurations of baffles in three different 1/a ratios and four fill levels. It would have taken a long time to finish this study if the model was not simplified. The 833 time steps were, therefore, reduced significantly to 35 points without any loss in the trend as shown in Figure 64.

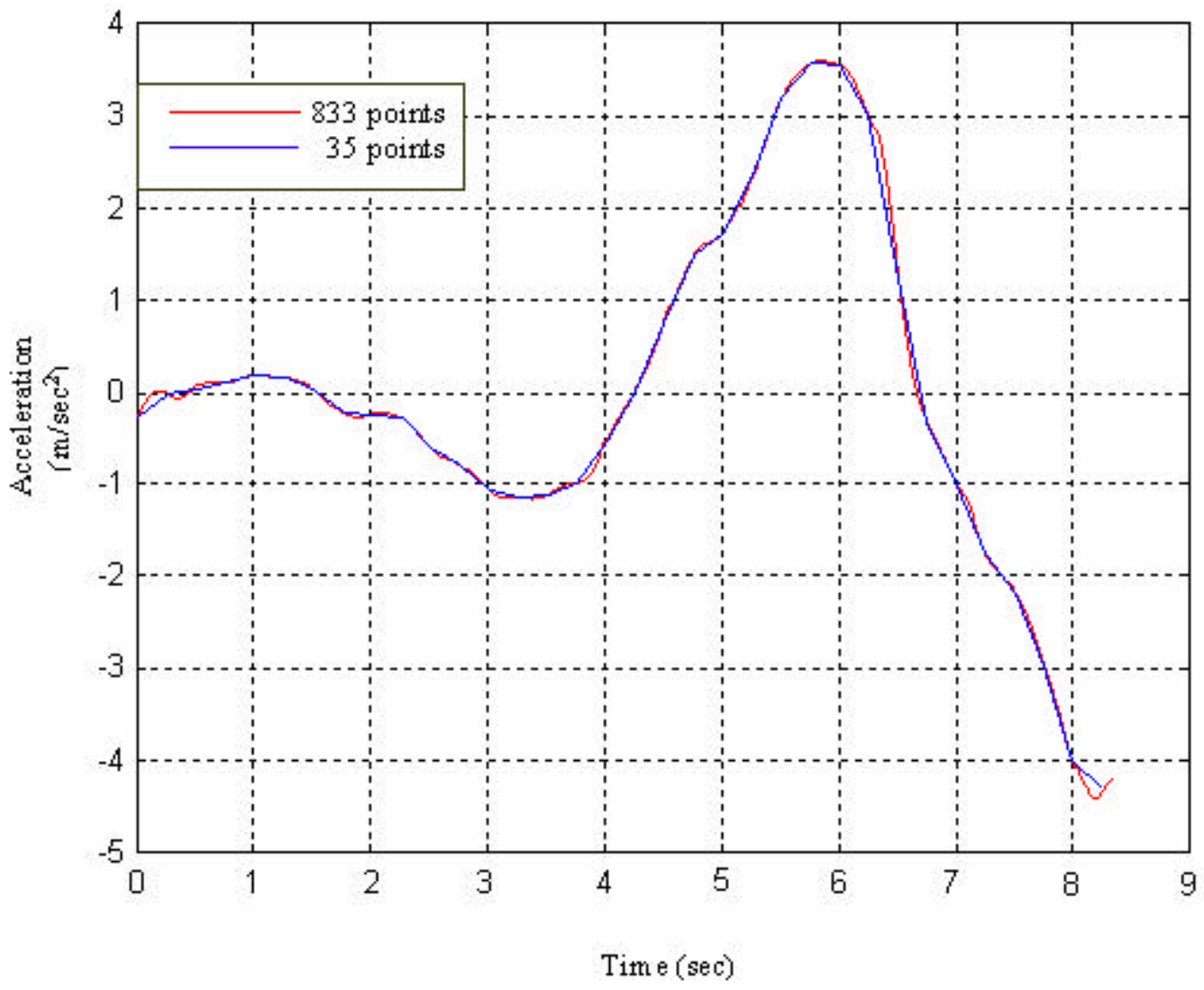

Figure 64. Comparison of actual data and reduced data 
The reduced data was in good agreement with the experimental data from ATC. The data that simulated the TOP lane change maneuver was used to feed the time varying accelerations into FLUENT. The results are discussed in the following two sections for circular and elliptical tanks.

\subsubsection{CIRCULAR TANK}

All the four configurations of baffles were analyzed to determine the effects of baffles in circular tanks maneuvering a double lane change maneuver. The data obtained above was used for the trailer lateral acceleration. Moments and forces on the tank walls were then obtained from above analyses. Dynamic coefficients of moment and force were then calculated from data files of moment and force.

The results in the case of a TOP lane change maneuver differed significantly from those of constant radius turn. Moments and sloshing were not reduced in all cases. In fact, horizontal baffles with $1 / \mathrm{a}$ ratios of 0.5 and 0.75 increased the moments and sloshing at higher levels. The results are shown in figures 65,66 and 67 . Baffles with $1 /$ a ratio of 0.25

reduced the peak moments rather insignificantly for fill levels greater than $50 \%$. Figure showing the percentage ratios of peak moments for all the baffle lengths (horizontal baffles) in this case of an elliptical tank to the peak moments in a tank without any baffles is shown in the Appendix A. 


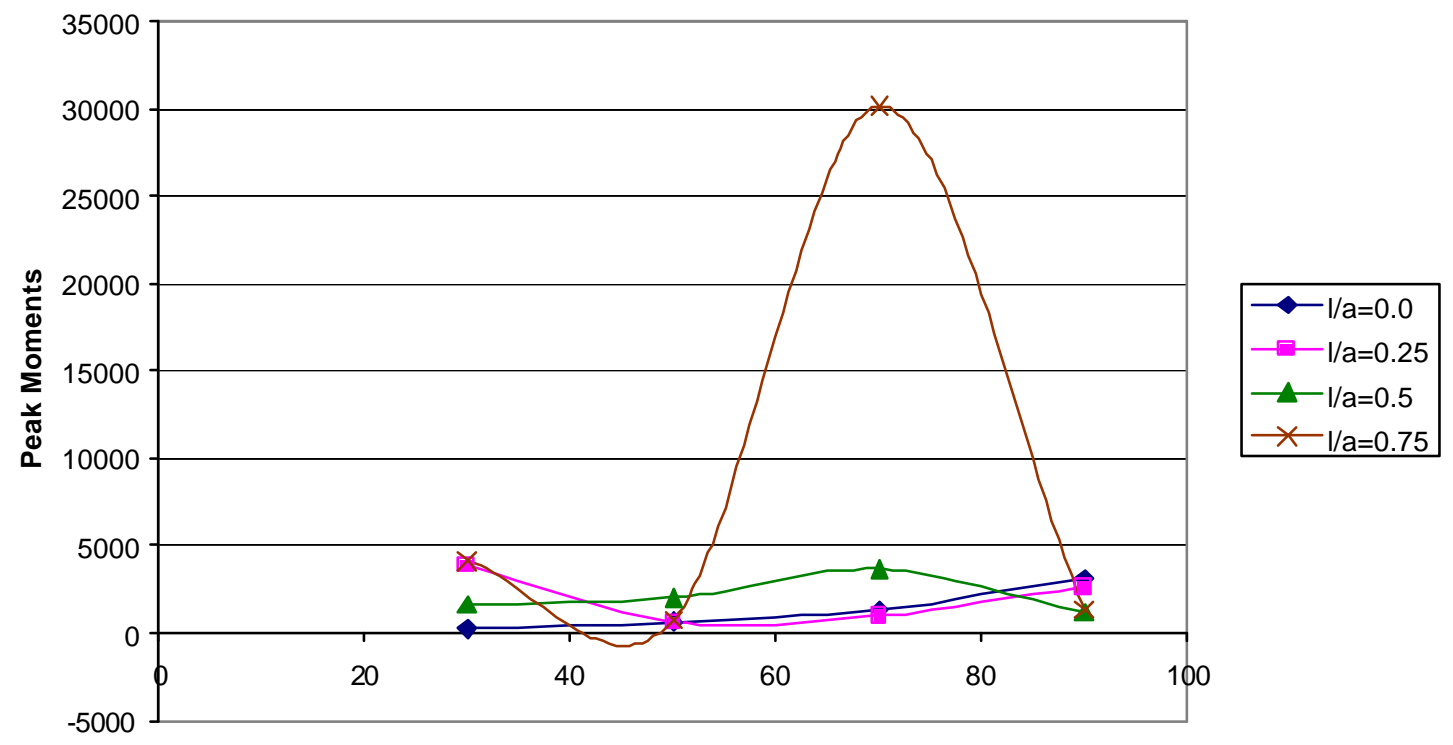

Fill Level

Figure 65. Peak moments for circular tank with horizontal baffles in a TOP lane change maneuver

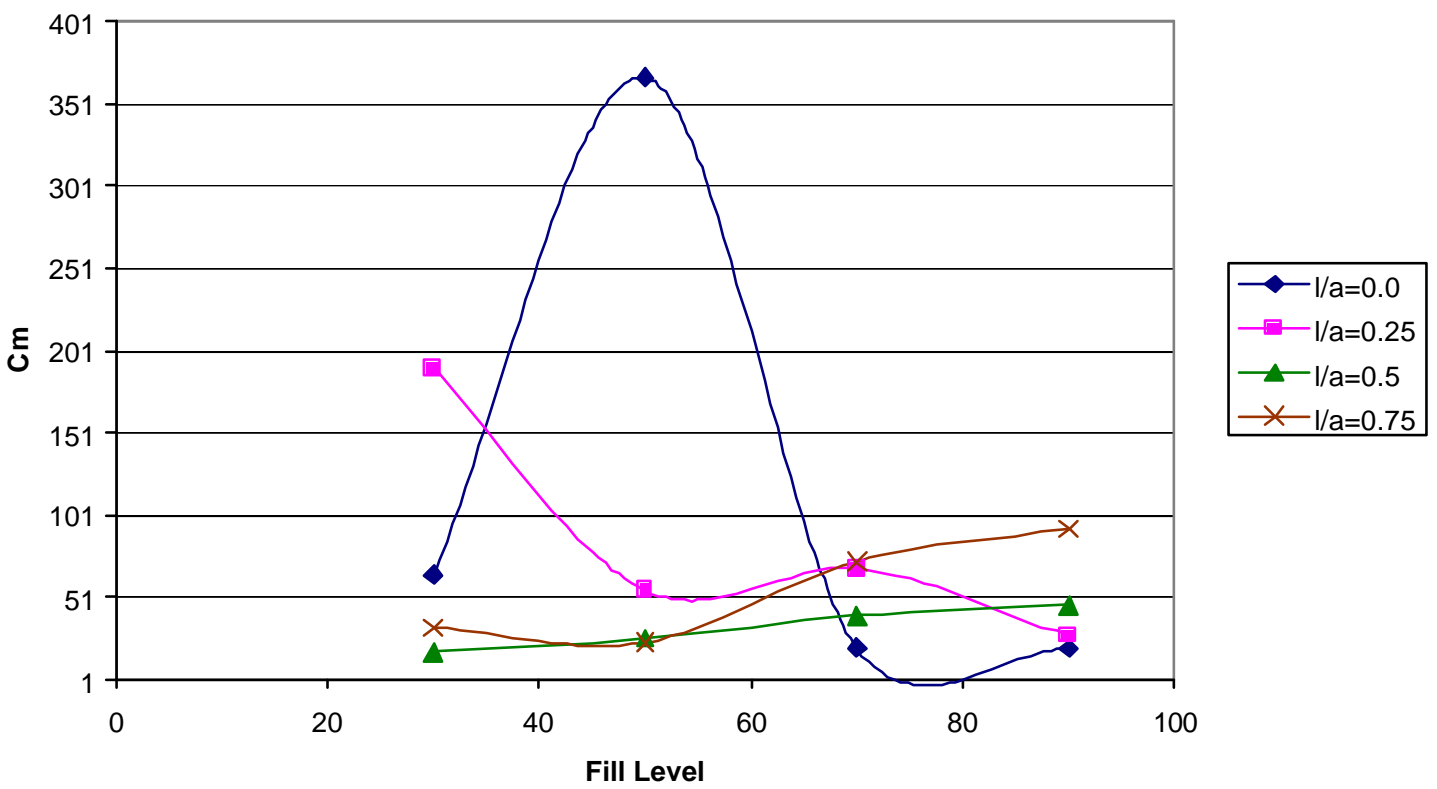

Figure 66. $\mathrm{C}_{\mathrm{m}}$ for circular tank with horizontal baffles in a TOP lane change maneuver 


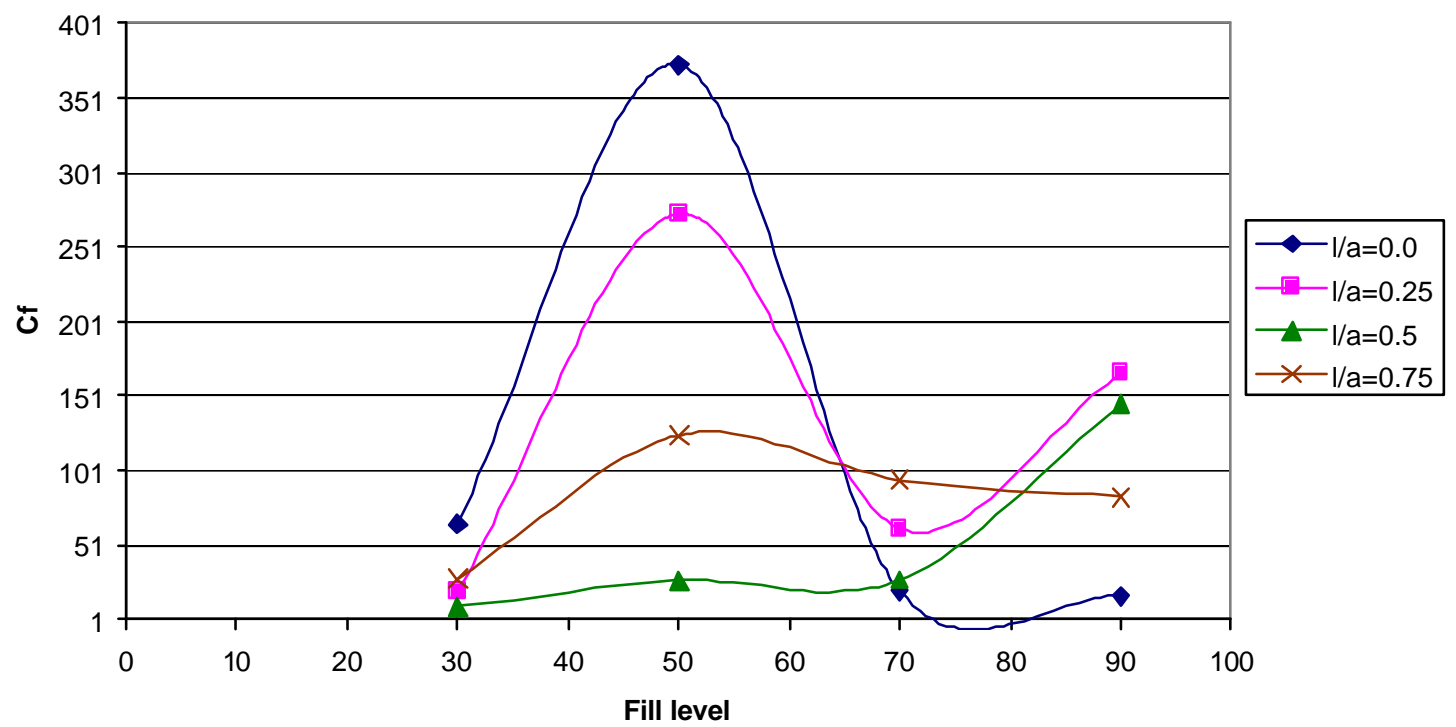

Figure $67 . \mathrm{C}_{\mathrm{f}}$ for circular tank with horizontal baffles in a TOP lane change maneuver

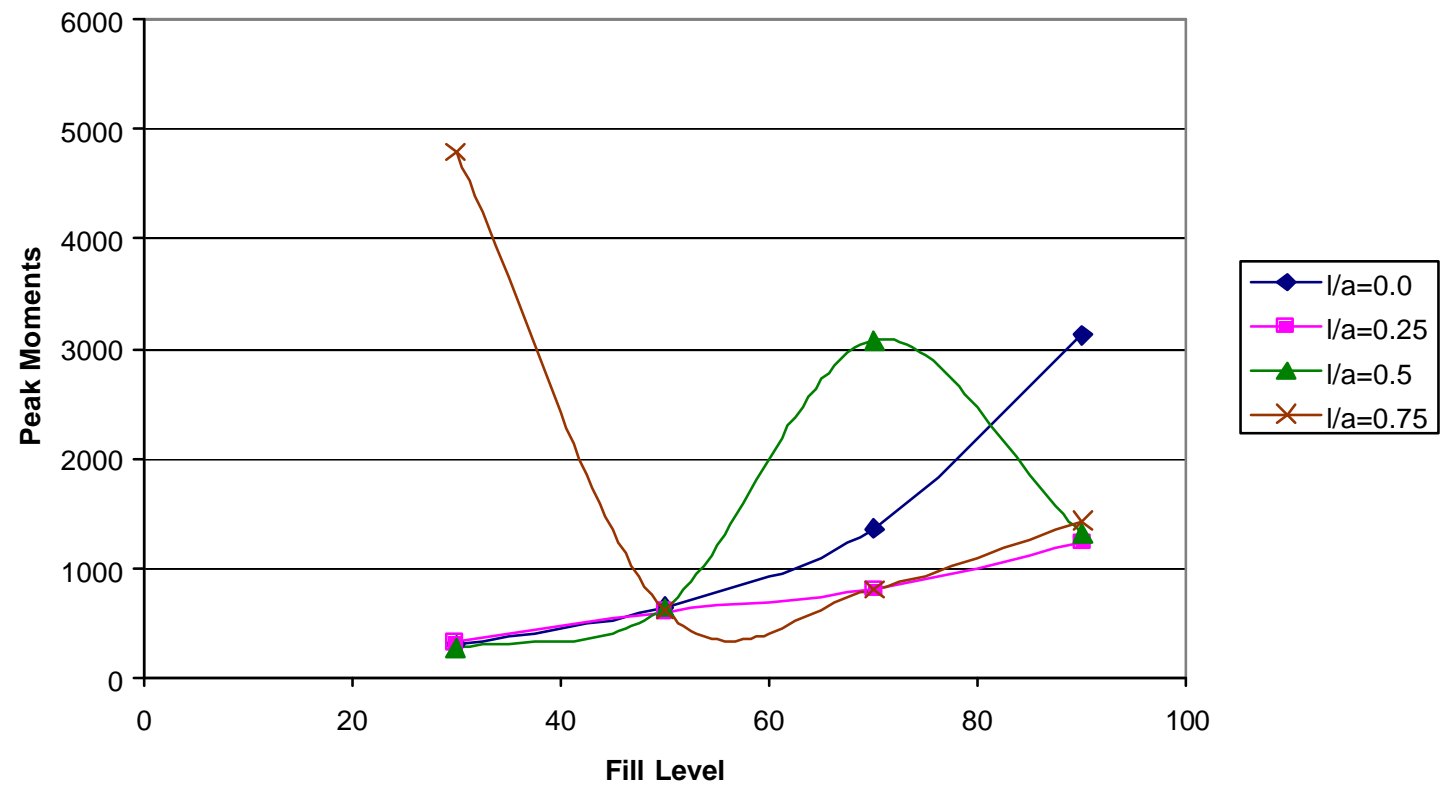

Figure 68. Peak moments for circular tank with vertical baffles in a TOP lane change maneuver 


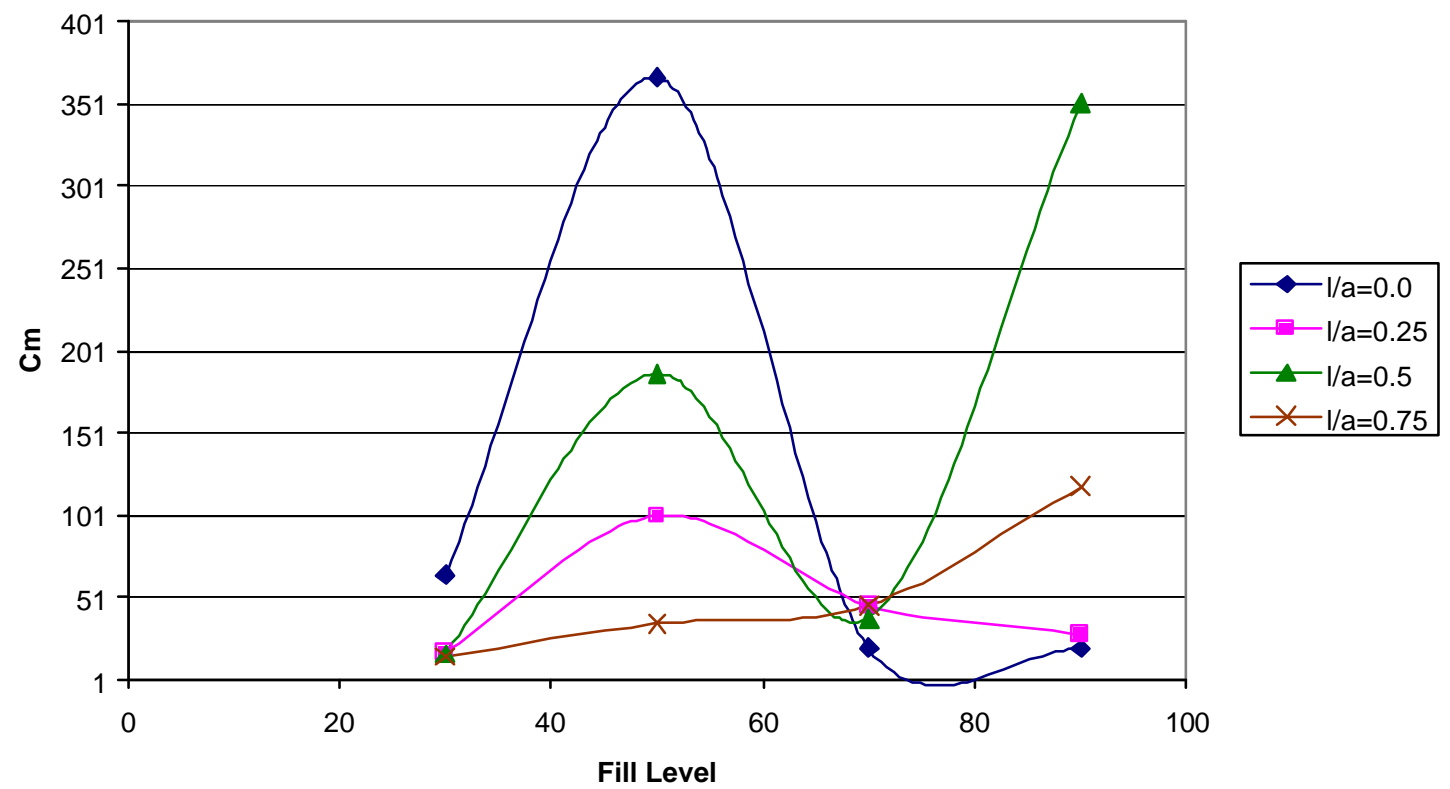

Figure 69. $C_{m}$ for circular tank with vertical baffles in a TOP lane change maneuver

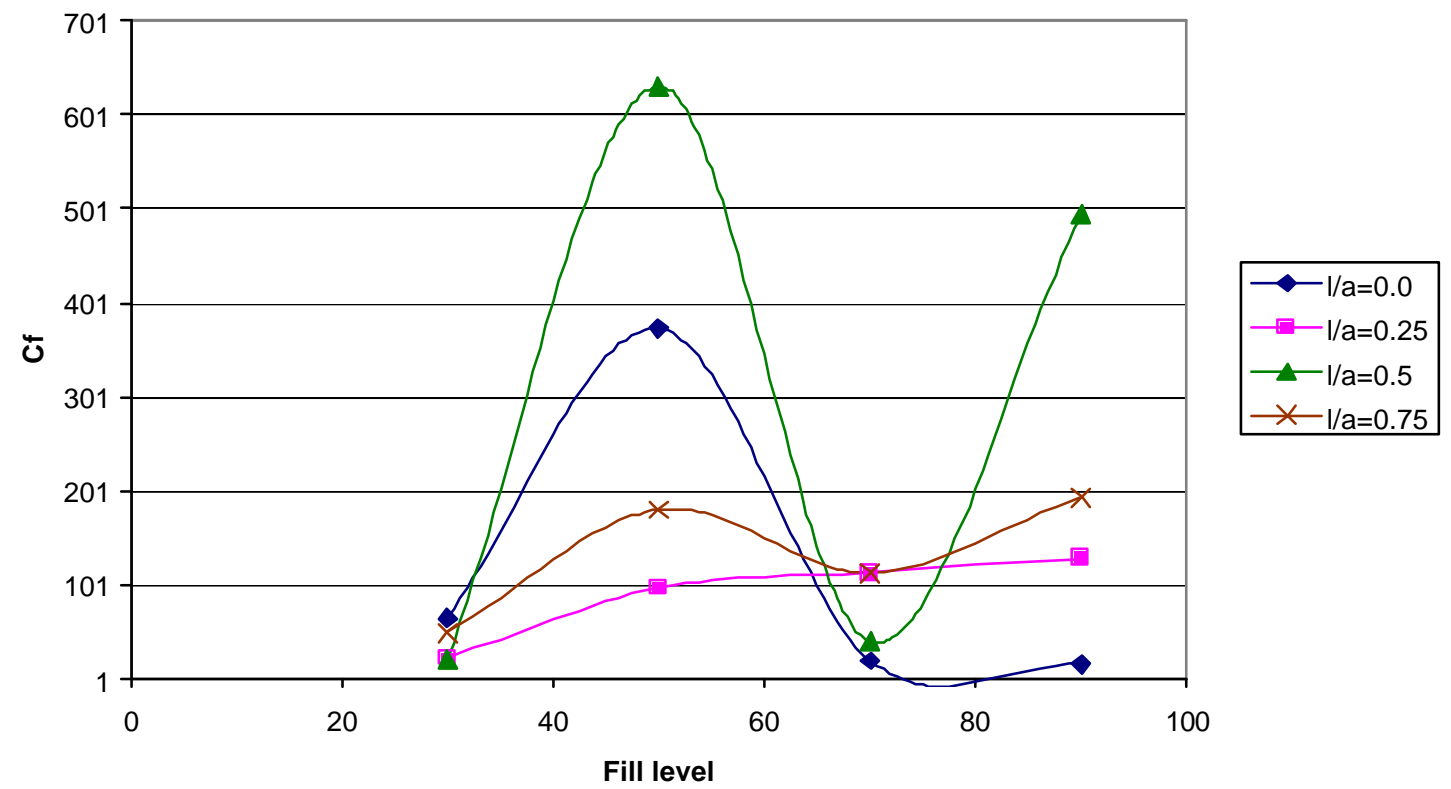

Figure $70 . \mathrm{C}_{\mathrm{f}}$ for circular tank with vertical baffles in a TOP Lane change maneuver 
Figures 68, 69 and 70 show that vertical baffles very much like the horizontal baffles were ineffective in reducing the peak moments for fill levels less than $50 \%$. However, vertical baffles do reduce sloshing effectively at lower levels of fill. Vertical baffles with $1 / \mathrm{a}$ ratios of 0.25 and 0.75 reduced the peak moments quite effectively at fill levels greater than 50\%. Interestingly they yielded a higher reduction at $90 \%$ fill level. A reduction of $54 \%$ was observed at a fill level of $90 \%$ with an 1/a ratio of 0.75 . Vertical baffles with $1 /$ a ratios of 0.25 resulted in a reduction of $60 \%$ at the $90 \%$ fill level.

Neither horizontal nor vertical baffles were found to be suitable for a circular tank in a TOP lane change maneuver. Although, vertical baffles were very effective at $90 \%$ fill level. Results of the horizontal-vertical baffle configuration are discussed next.

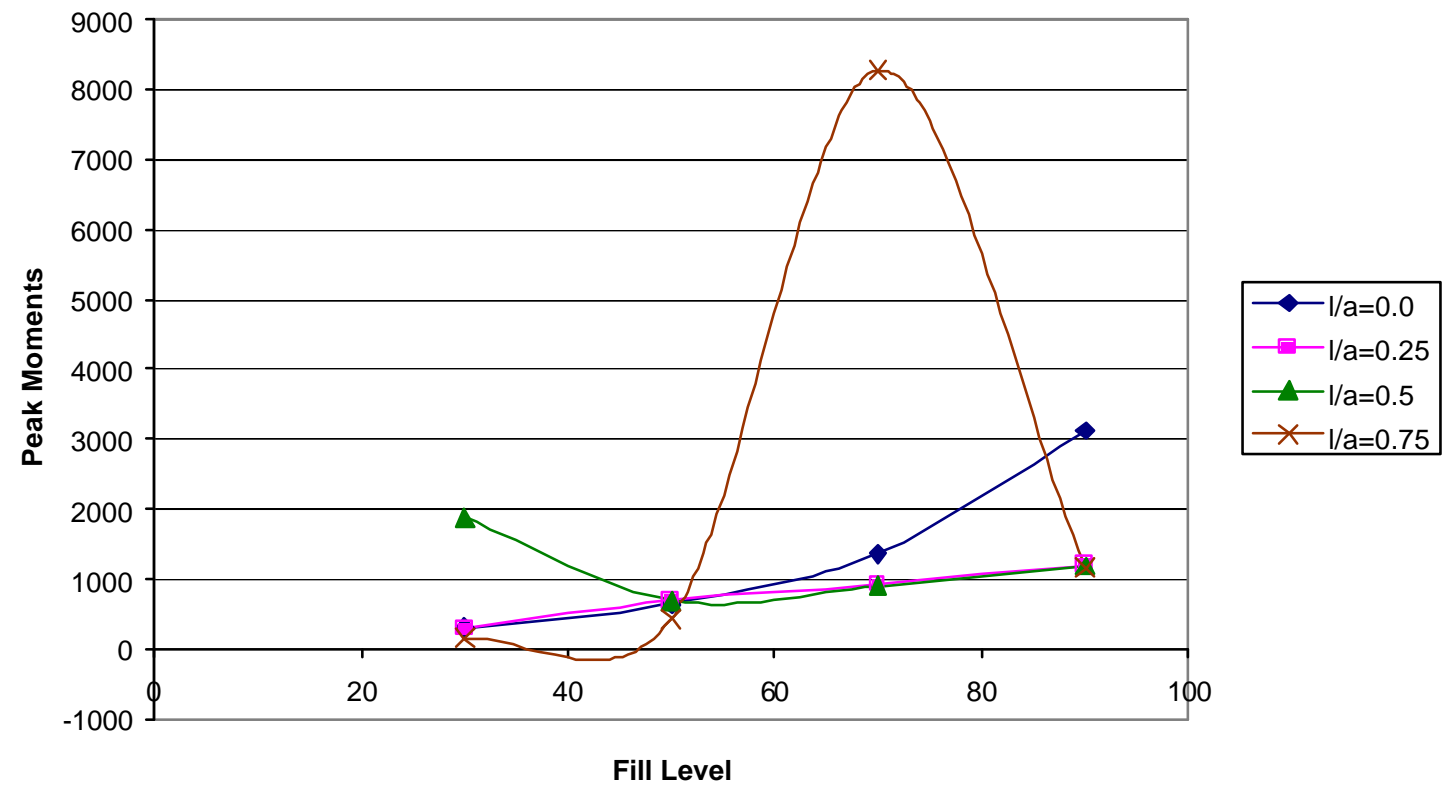

Figure 71. Peak moments for circular tank with horizontal-vertical baffles in a TOP lane change maneuver 


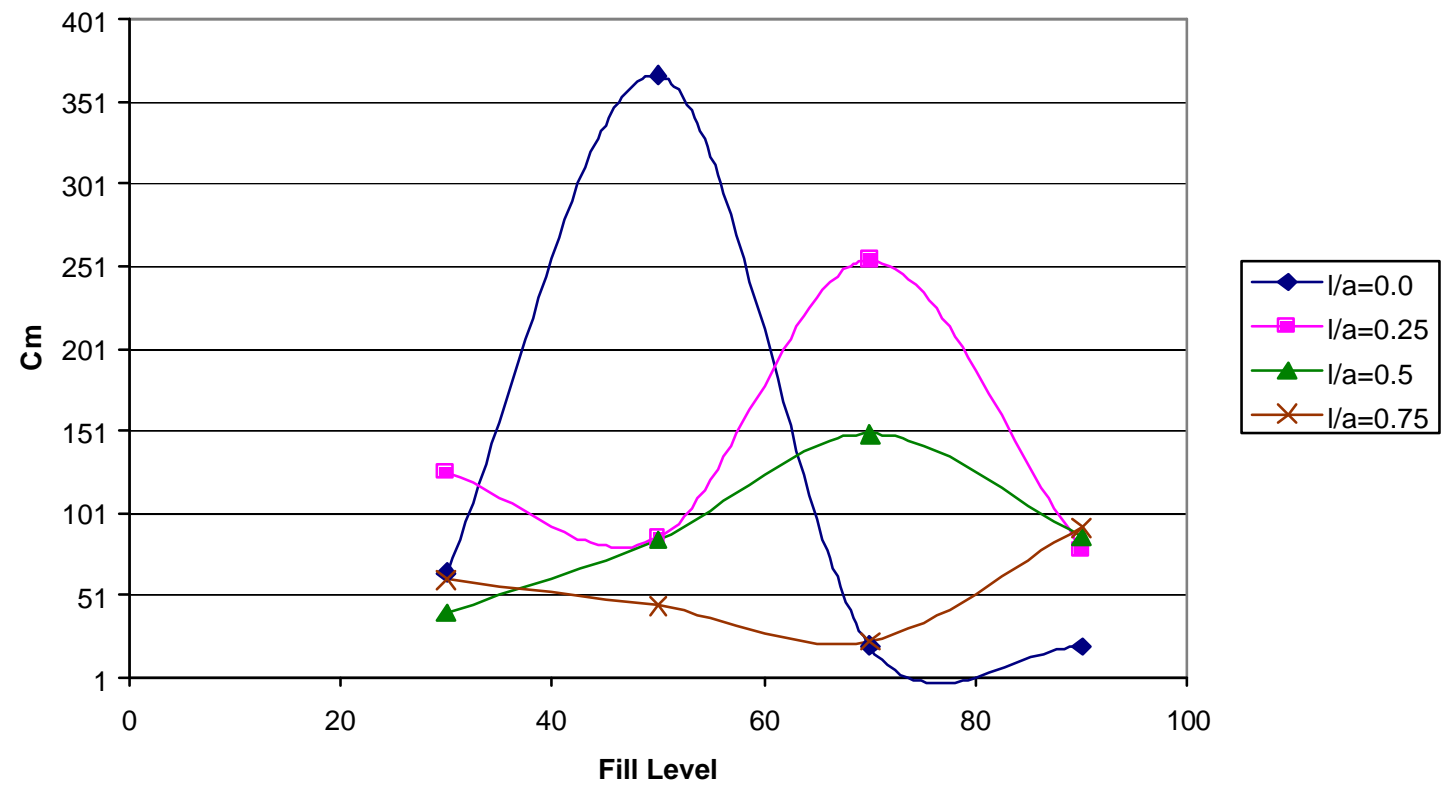

Figure 72. $\mathrm{C}_{\mathrm{m}}$ for circular tank with horizontal-vertical baffles in a TOP lane change maneuver

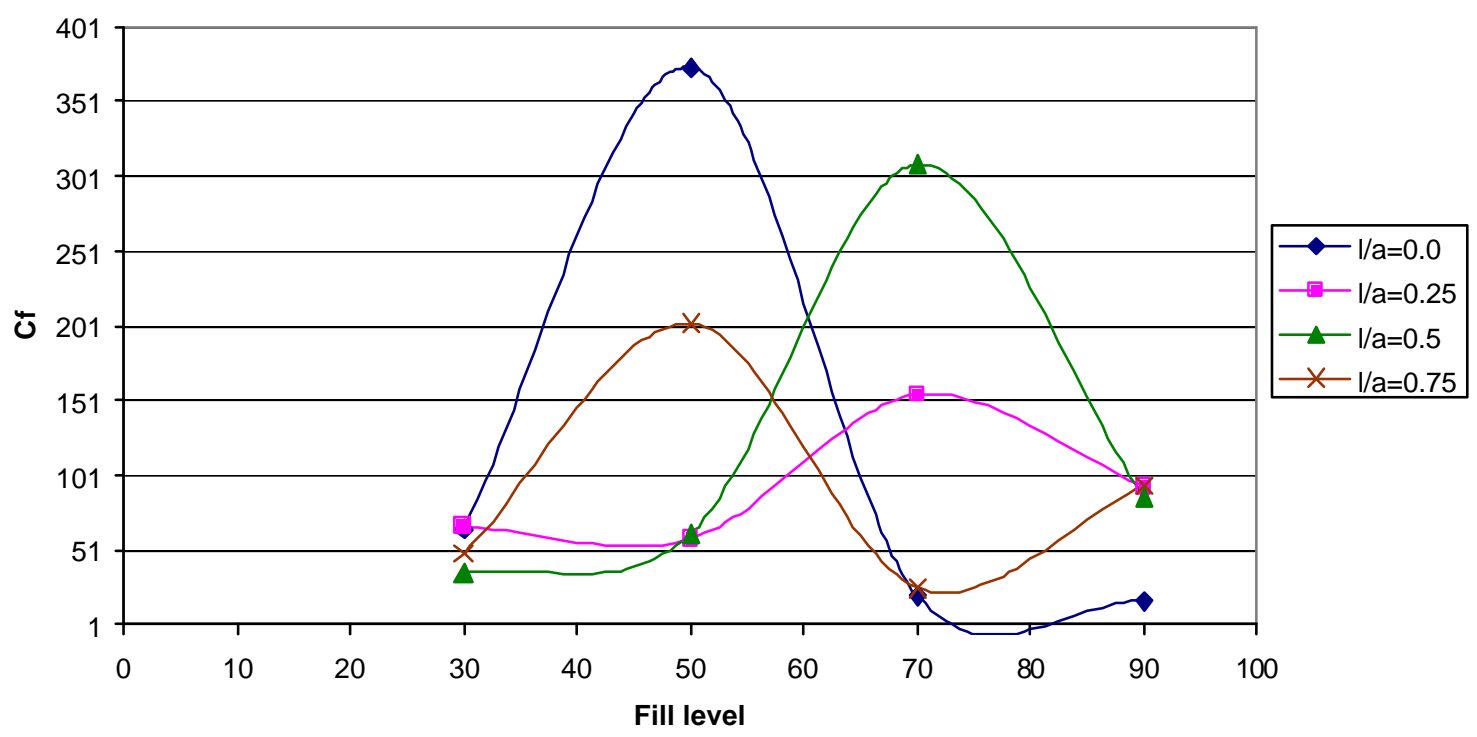

Figure 73. $\mathrm{C}_{\mathrm{f}}$ for circular tank with horizontal-vertical baffles in a TOP lane change maneuver 
The configuration of horizontal-vertical baffles behaved in the same manner as the two configurations discussed earlier. It is evident from figures 71,72 and 73 . There was no advantage at fill levels less than $50 \%$. However, they do seem effective at higher fill levels, especially at the $90 \%$ fill level. It should be noted that this baffle configuration increased sloshing at these higher levels. More important than sloshing is the peak moment, which determines the rollover threshold velocity. Hence the reduction in moments at higher fill levels makes the configuration of horizontal-vertical baffles rather desirable at higher levels of fluid in the case of a circular tank undergoing a TOP lane change maneuver. Figure showing the percentage ratios of peak moments for all the baffle lengths (horizontal-vertical baffles) in this case of an elliptical tank to the peak moments in a tank without any baffles is shown in the Appendix A.

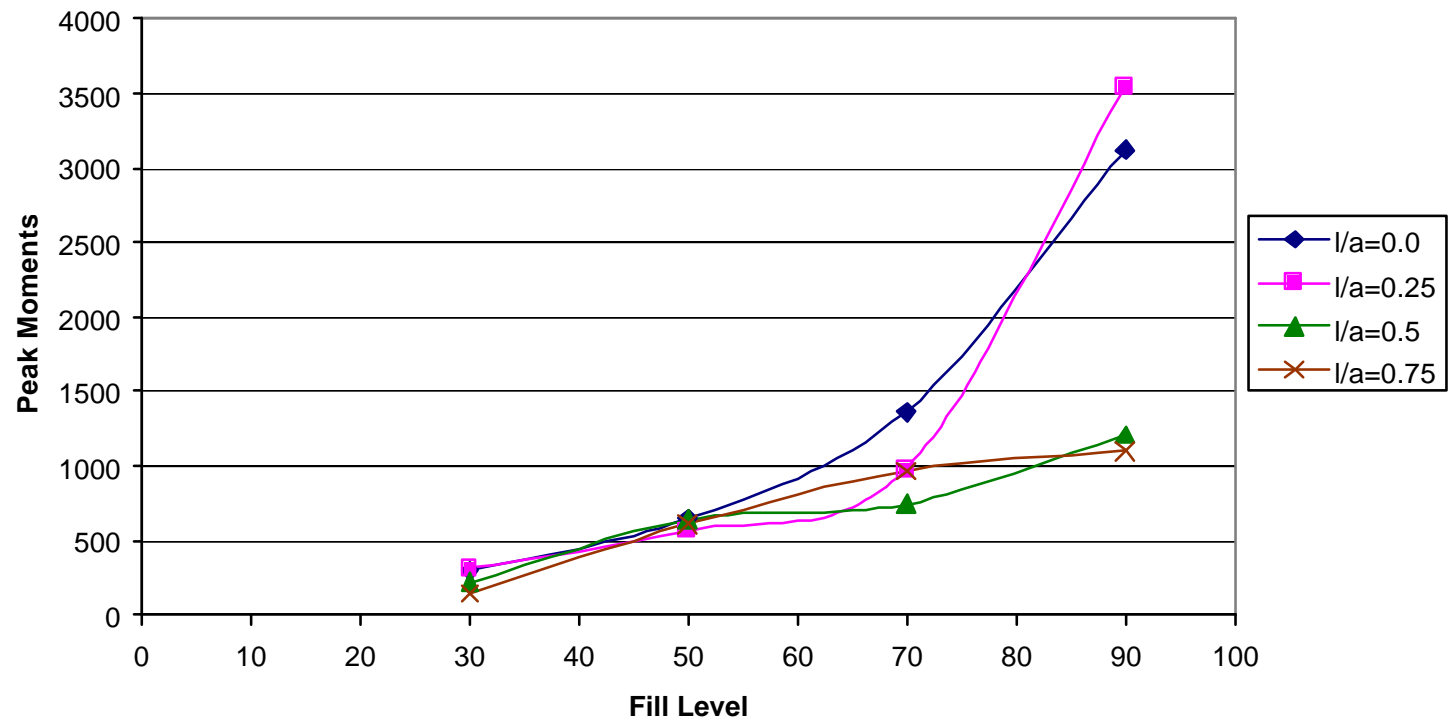

Figure 74. Peak moments for circular tank with diagonal baffles in a TOP lane change maneuver 


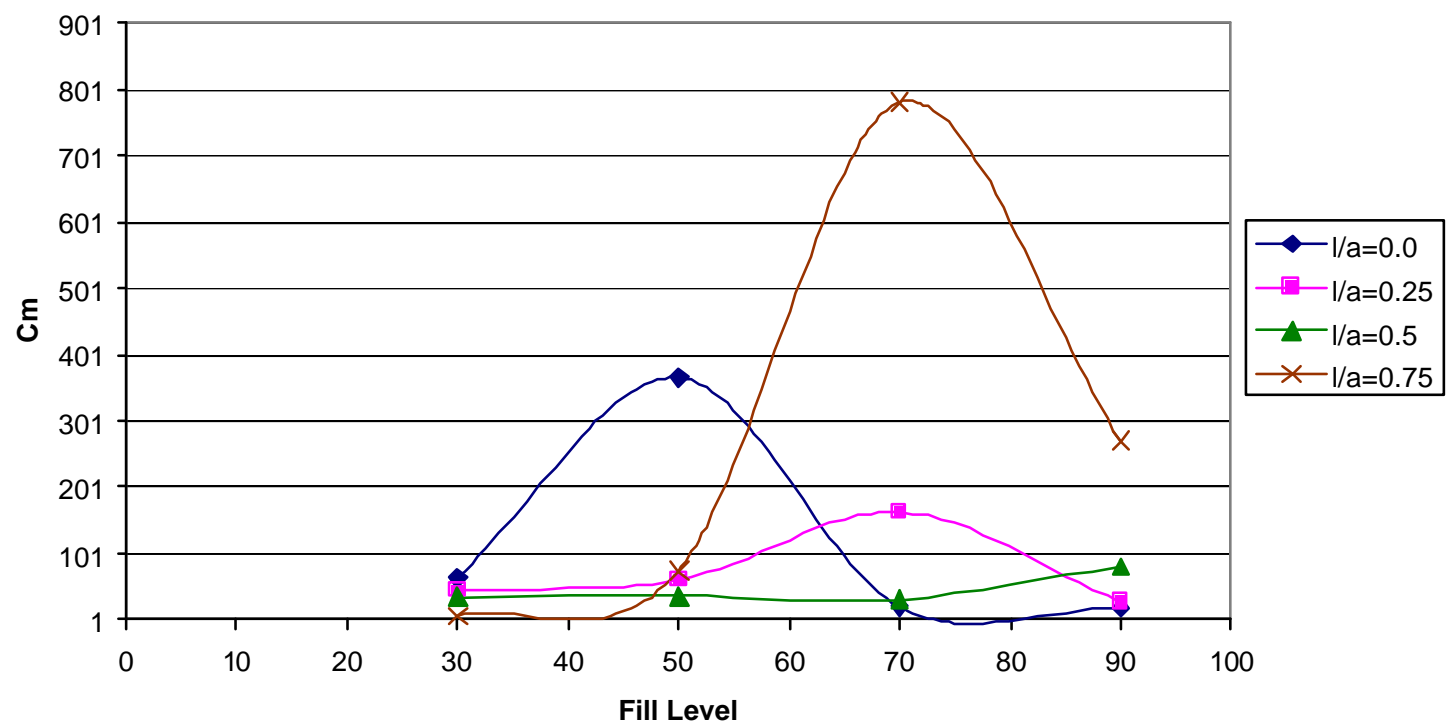

Figure $75 . \mathrm{C}_{\mathrm{m}}$ for circular tank with diagonal baffles in a TOP lane change maneuver

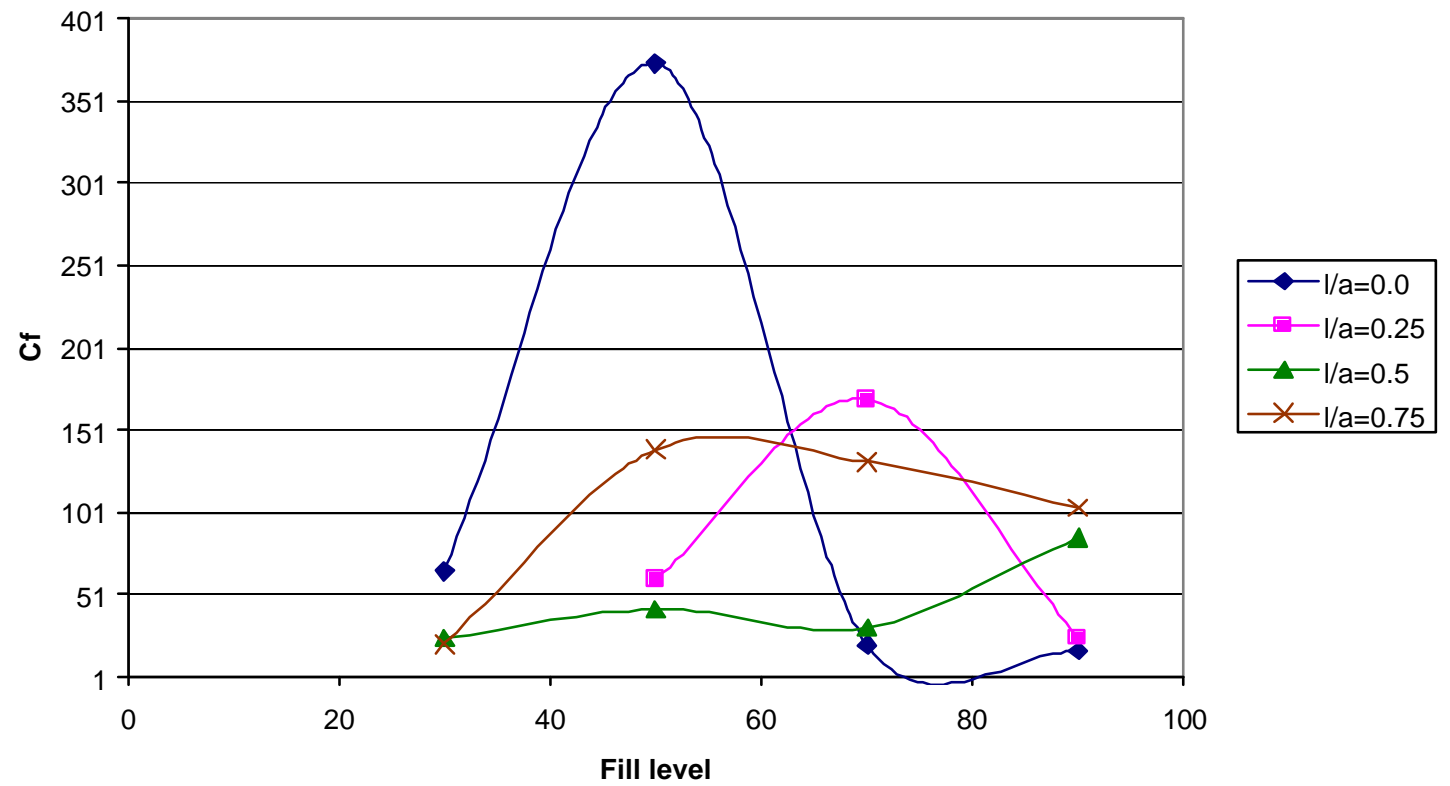

Figure $76 . \mathrm{C}_{\mathrm{f}}$ for circular tank with diagonal baffles in a TOP lane change maneuver

Figures 74, 75 and 76 show the peak moments and dynamic coefficients for a circular tank with diagonal baffles traveling through a TOP lane change maneuver. From 
these figures diagonal baffles were found to be the best configuration of baffles for a circular tank undergoing a TOP lane change maneuver. There is a reduction in the peak moments for all fill levels, although the reduction for fill levels less than $50 \%$ is very negligible. Again, baffles increased sloshing for fill levels greater than 50\%. Baffles with 1/a ratios of 0.5 and 0.75 resulted in a reduction of $64 \%$ in peak moments at a $90 \%$ fill level. The reduction at fill 70 is $28.9 \%$, and this is considered to be a significant reduction.

Diagonal baffles appeared to be a better configuration than horizontal baffles, vertical baffles or horizontal-vertical, for a circular tank.

\subsubsection{ELLIPTICAL TANK}

Figures 77, 78 and 79 show the peak moments and dynamic coefficients for an elliptical tank with horizontal baffles undergoing a TOP lane change maneuver. As can be seen in Figure 77, horizontal baffles had no effect in reducing the moments in an elliptical tank undergoing a TOP lane change maneuver similar to the case of a circular tank. However, 1/a ratios of 0.25 and 0.75 in horizontal baffles did yield some reduction (about 15\%) for fill levels greater than 50\%. An 1/a ratio of 0.5 showed a reduction in peak moments only at $50 \%$ fill level due to the damping at the free surface of the fluid.

Vertical baffles were also found to be ineffective in reducing the peak moments. Figures 80,81 and 82 show the effects of vertical baffles in an elliptical tank undergoing a TOP lane change maneuver. An 1/a ratio of 0.75 was found to be more effective for fill 
levels less than $50 \%$. For fill levels greater than $50 \%$ none of the vertical baffles were effective.

Horizontal-vertical baffles yielded good results with reduction in moments for all fill levels. A reduction of $48 \%$ for a $30 \%$ fill level, $56 \%$ for $50 \%$ fill, $21 \%$ for $70 \%$ fill, and $25 \%$ for $90 \%$ fill level were observed with these baffles. Effects of horizontalvertical baffles are shown in figures 83,84 and 85 .

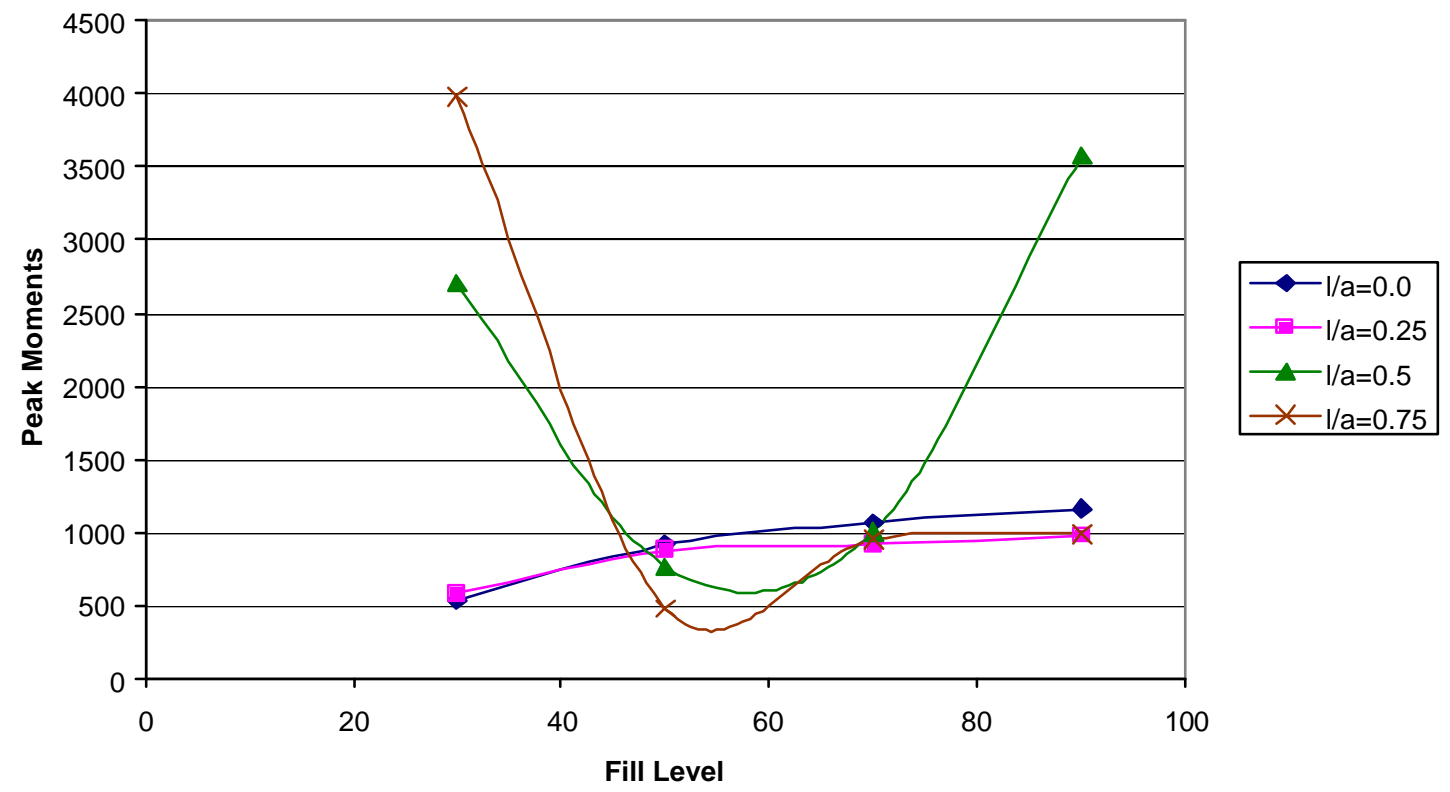

Figure 77. Peak moments for elliptical tank with horizontal baffles in a TOP lane change maneuver 


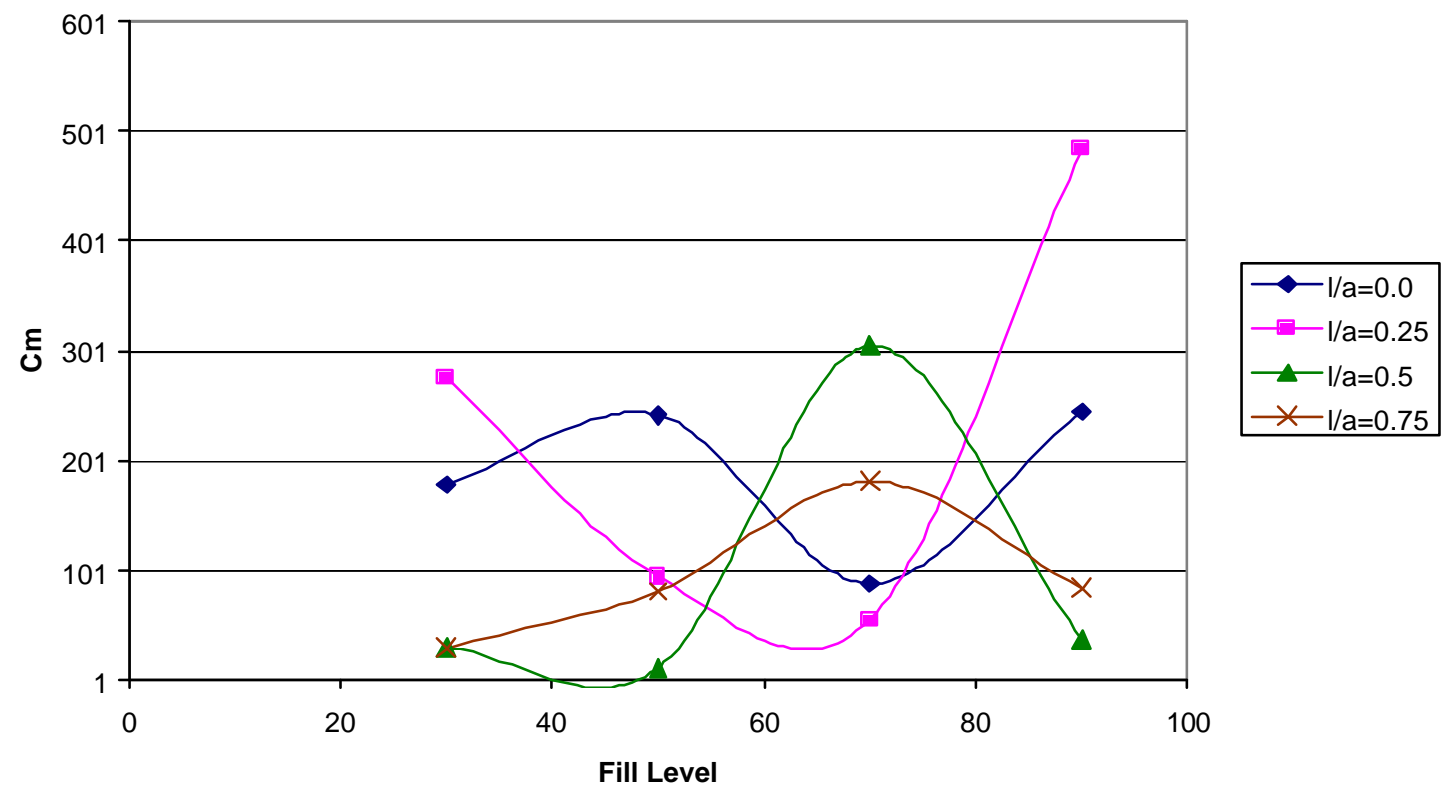

Figure 78. $\mathrm{C}_{\mathrm{m}}$ for elliptical tank with horizontal baffles in a TOP lane change maneuver

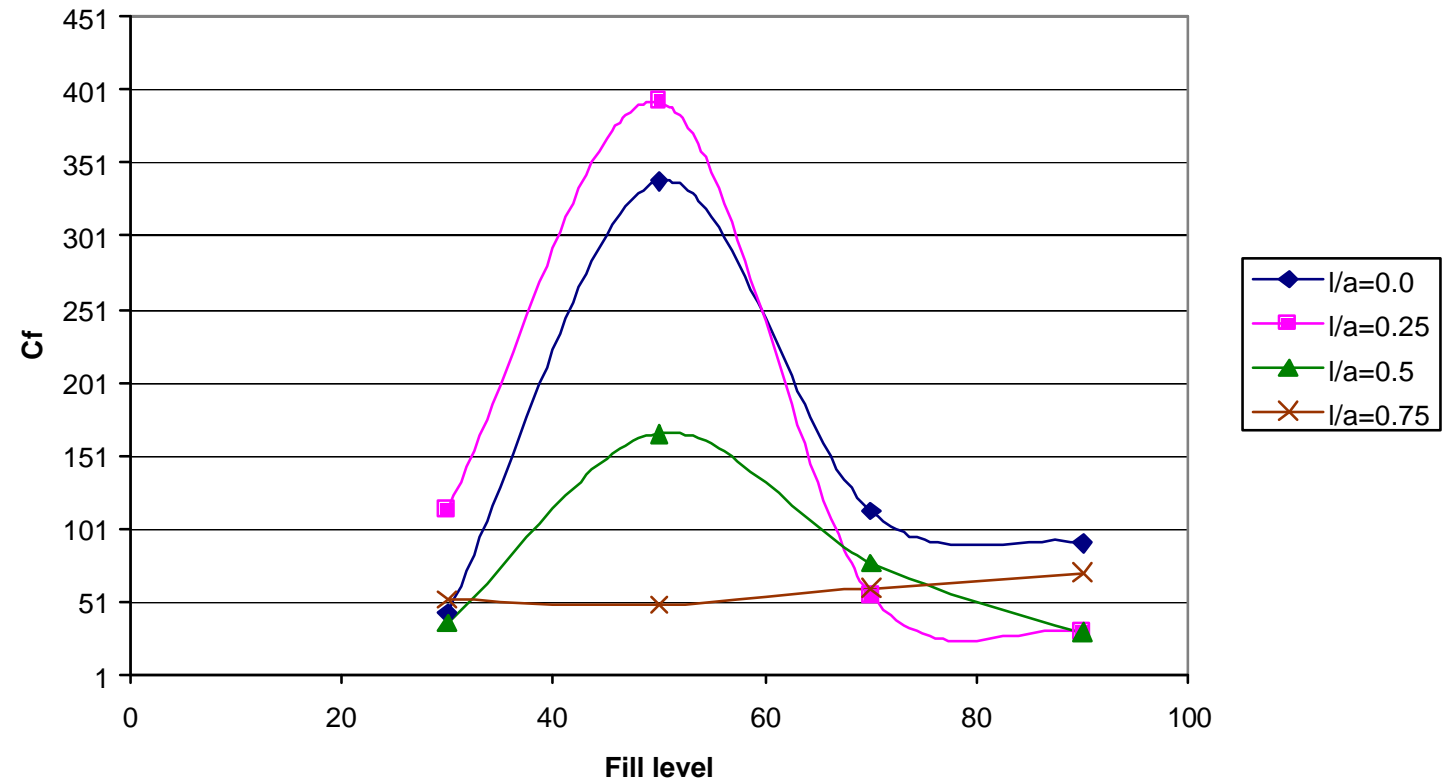

Figure 79. $\mathrm{C}_{\mathrm{f}}$ for elliptical tank with horizontal baffles in a TOP lane change maneuver 


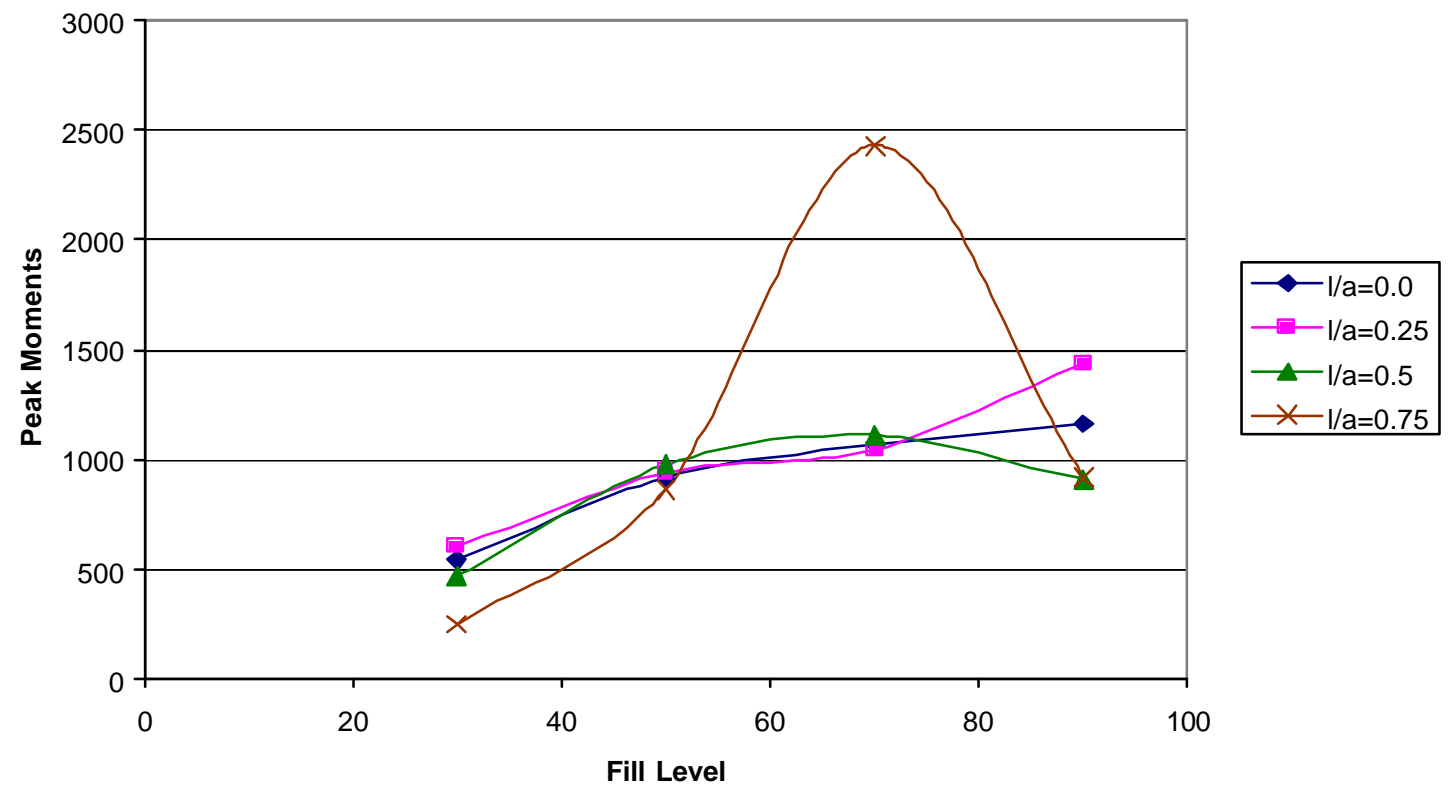

Figure 80. Peak moments for elliptical tank with vertical baffles in a TOP lane change maneuver

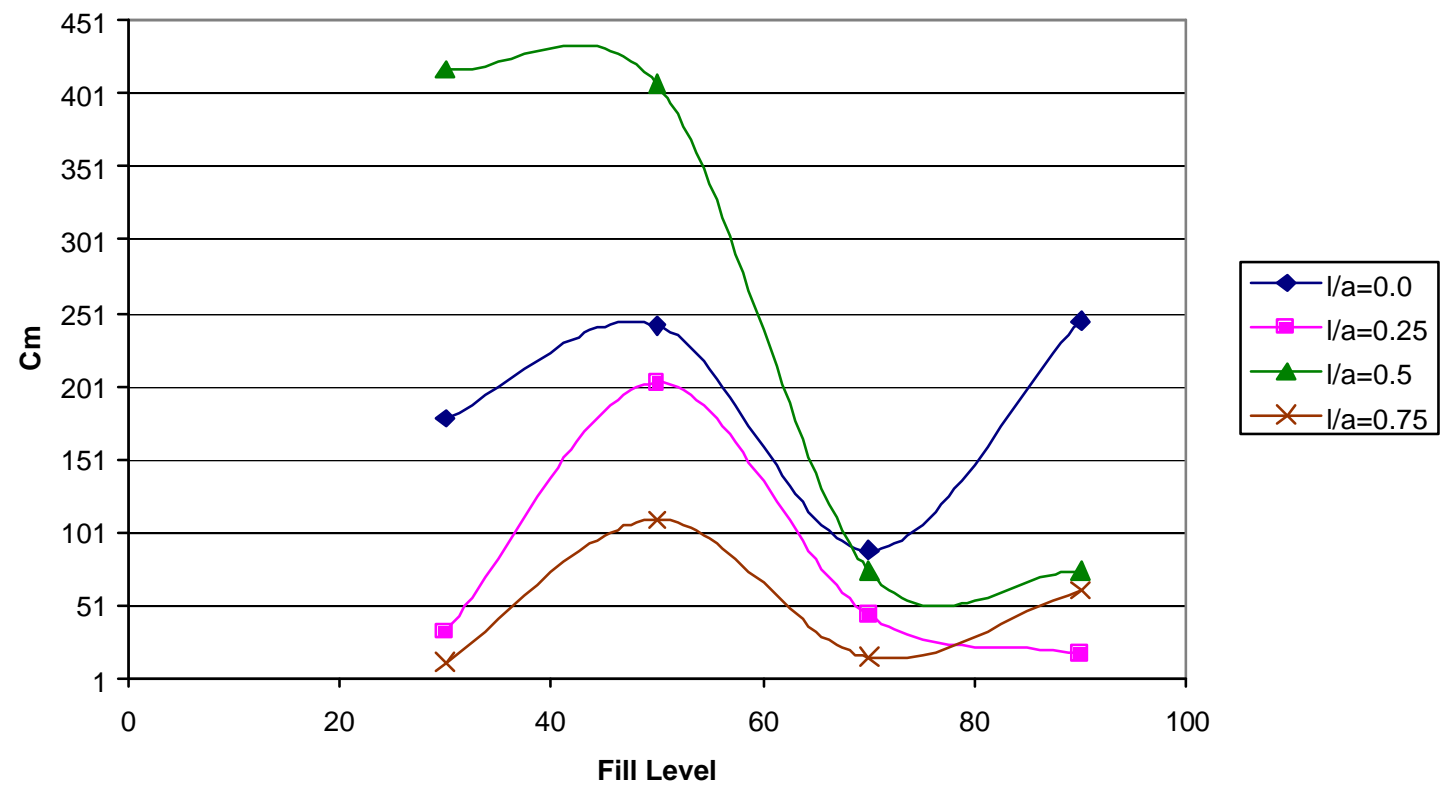

Figure $81 . \mathrm{C}_{\mathrm{m}}$ for elliptical tank with vertical baffles in a TOP lane change maneuver 


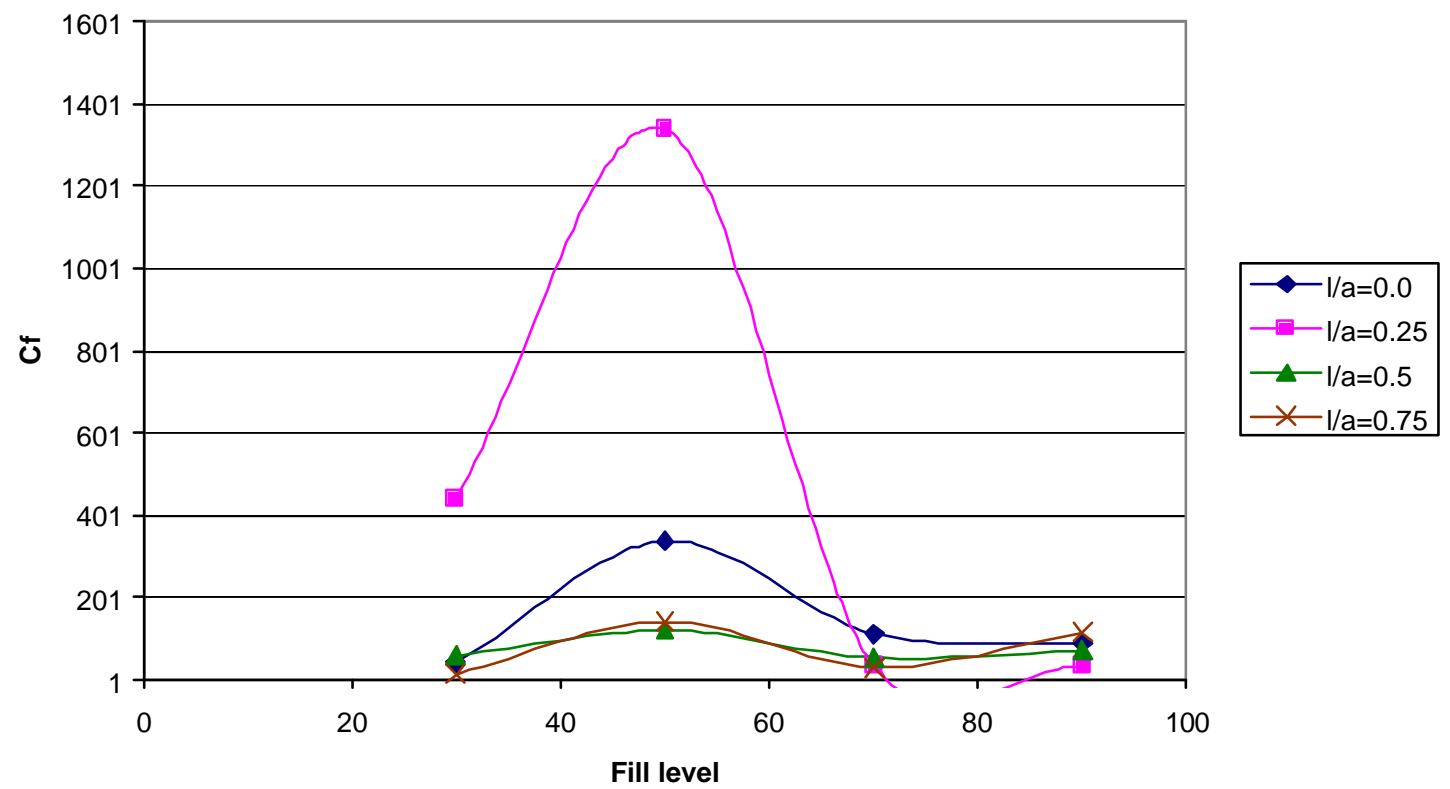

Figure $82 . \mathrm{C}_{\mathrm{f}}$ for elliptical tank with vertical baffles in a TOP lane change maneuver

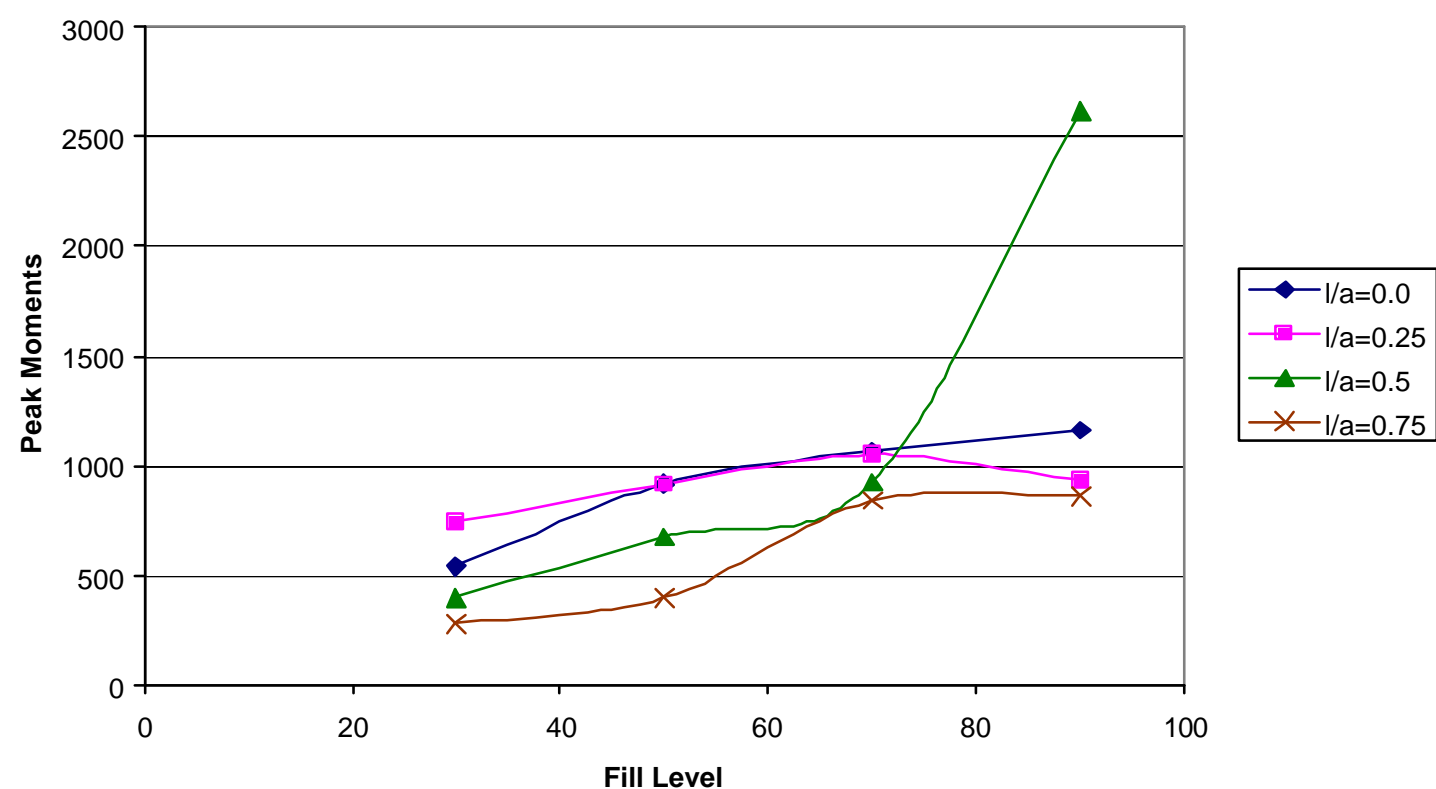

Figure 83. Peak moments for elliptical tank with horizontal-vertical baffles in a TOP lane change maneuver 


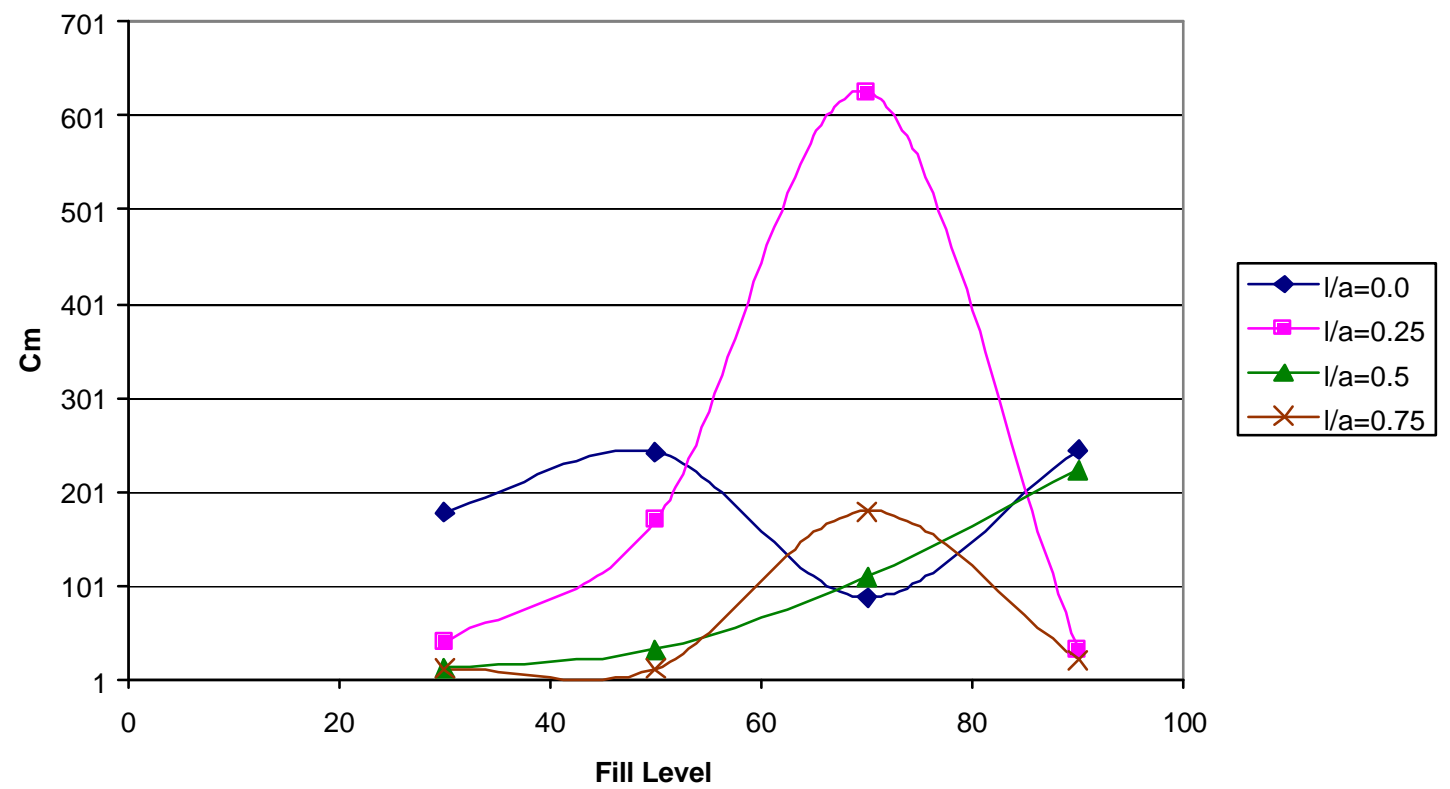

Figure $84 . \mathrm{C}_{\mathrm{m}}$ for elliptical tank with horizontal-vertical baffles in a TOP lane change maneuver

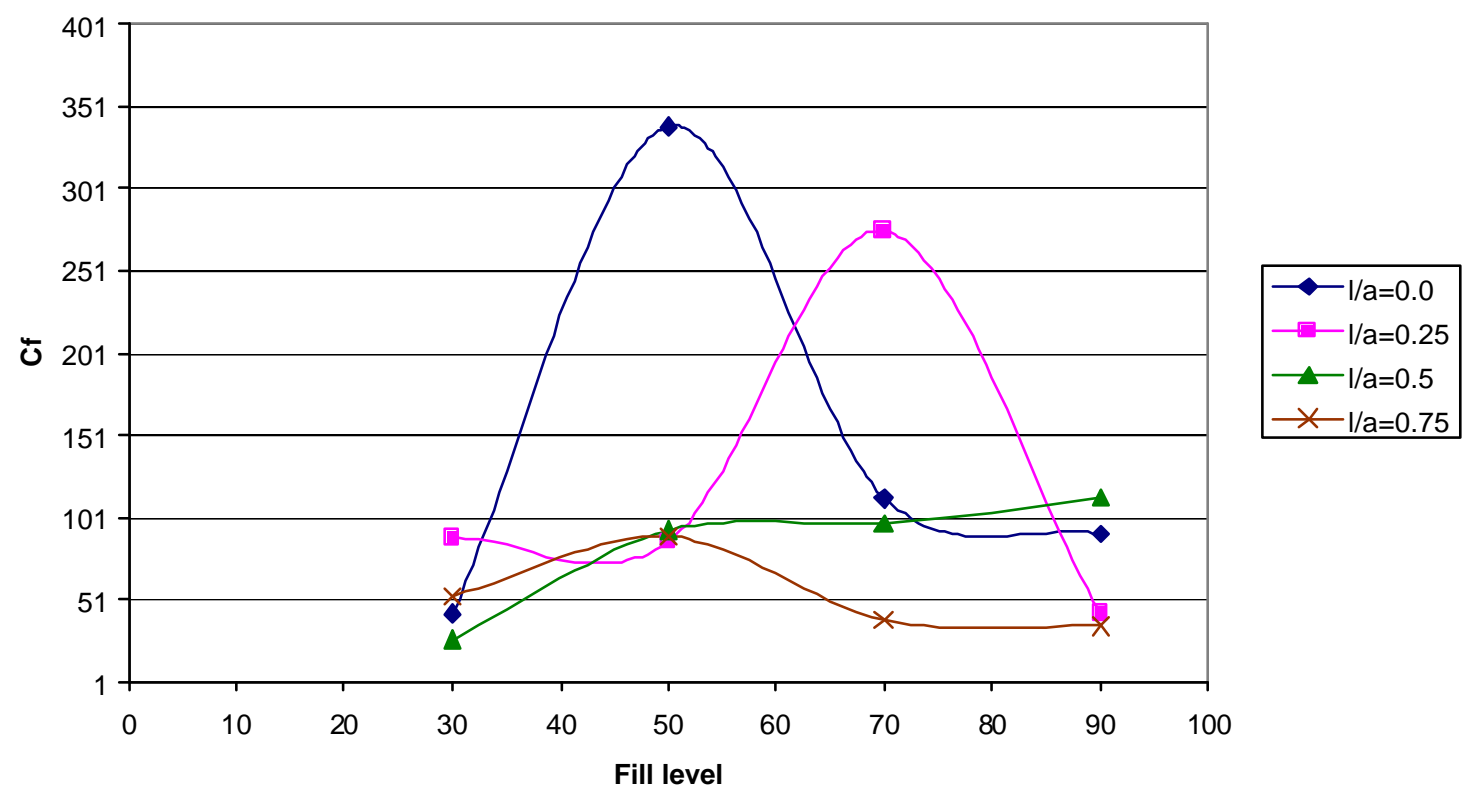

Figure $85 . \mathrm{C}_{\mathrm{f}}$ for elliptical tank with horizontal-vertical baffles in a TOP lane change maneuver 
Effects of diagonal baffles in an elliptical tank undergoing a TOP lane change maneuver are shown in figures 86, 87 and 88. Diagonal baffles effectively reduced sloshing for all fill levels. However, these diagonal baffles were unable to reduce the peak moments for fill levels greater than $50 \%$. For fill levels less than $50 \% 1 / \mathrm{a}$ ratios of 0.5 and 0.75 did yield little reduction in peak moments with maximum reduction of approximately $60 \%$ at a fill level of $50 \%$.

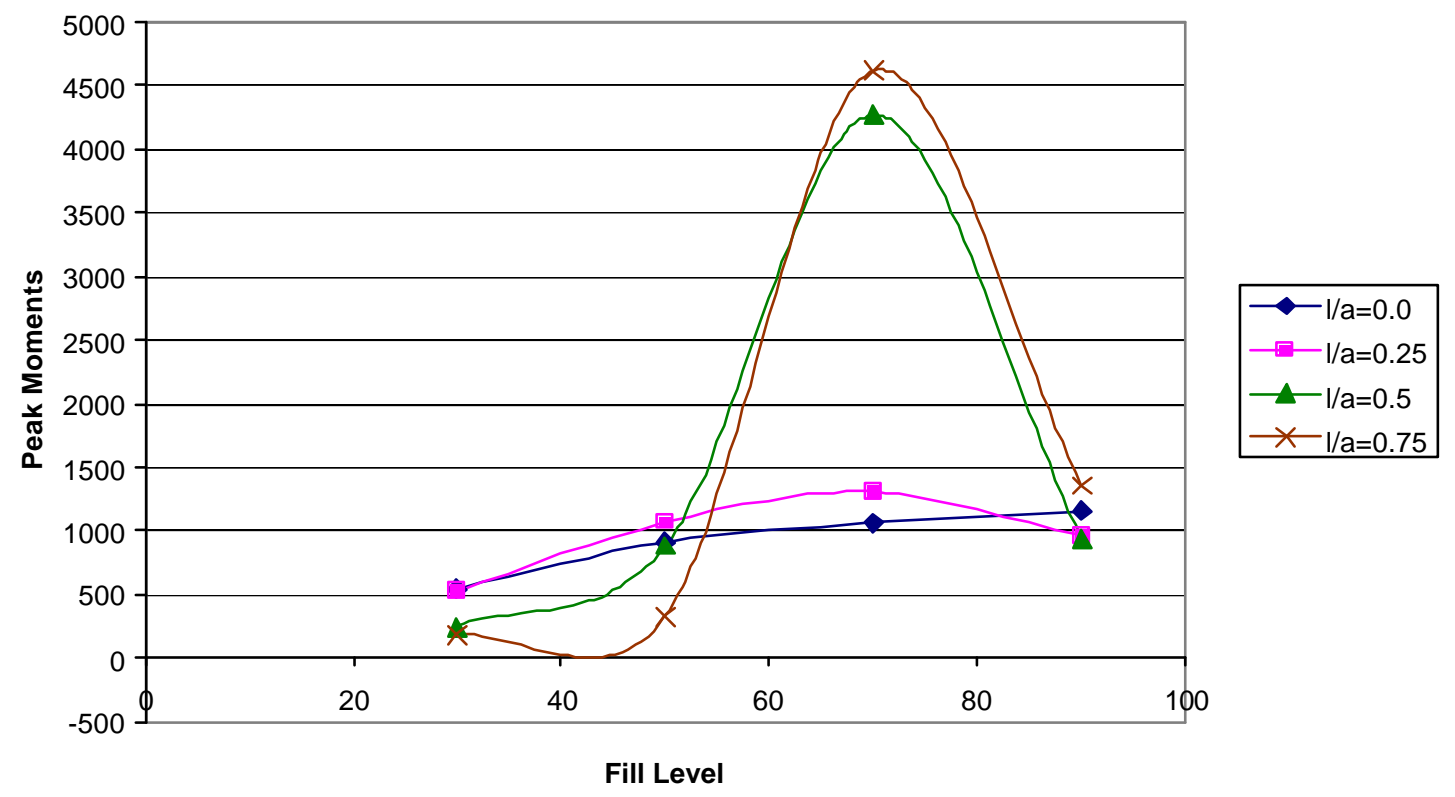

Figure 86. Peak moments for elliptical tank with diagonal baffles in a TOP lane change maneuver 


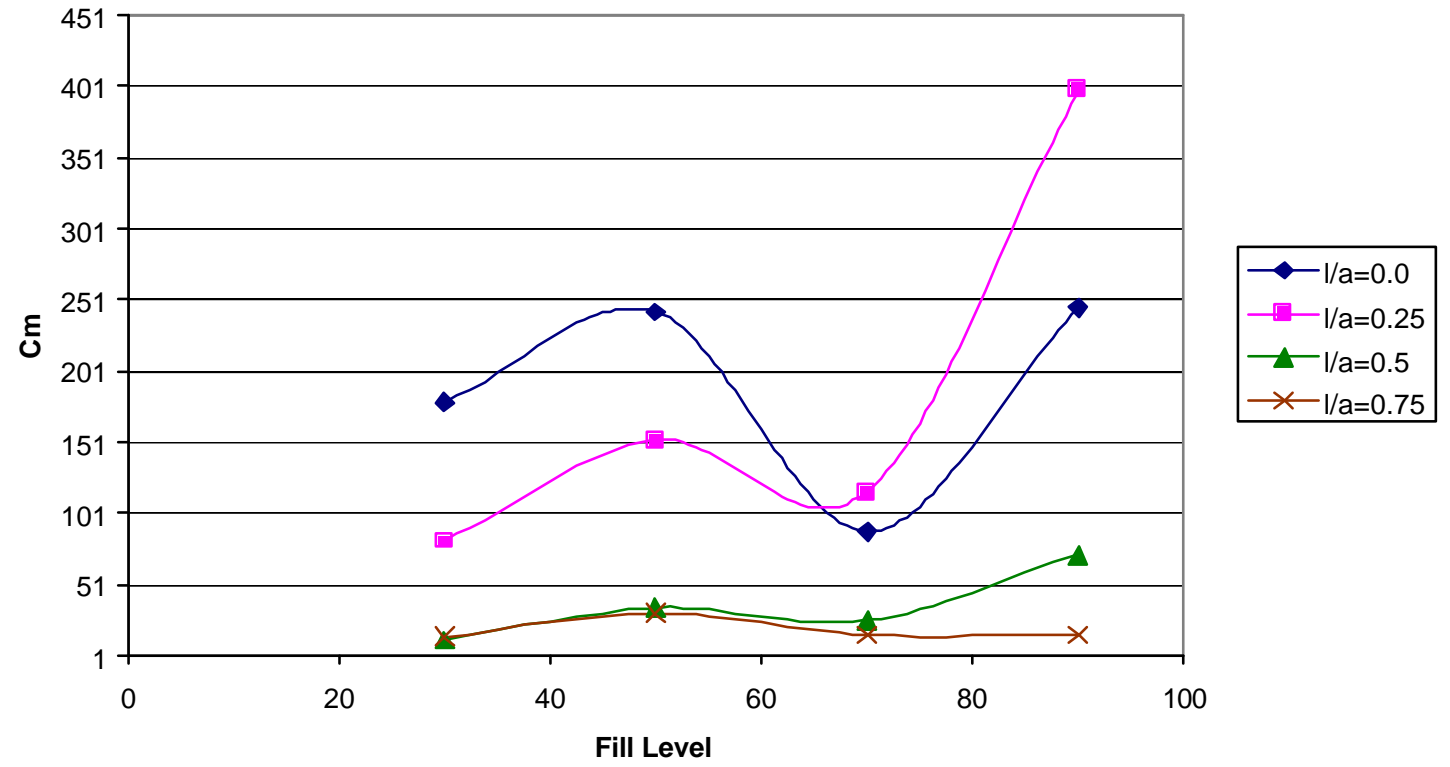

Figure $87 . \mathrm{C}_{\mathrm{m}}$ for elliptical tank with diagonal baffles in a TOP lane change maneuver

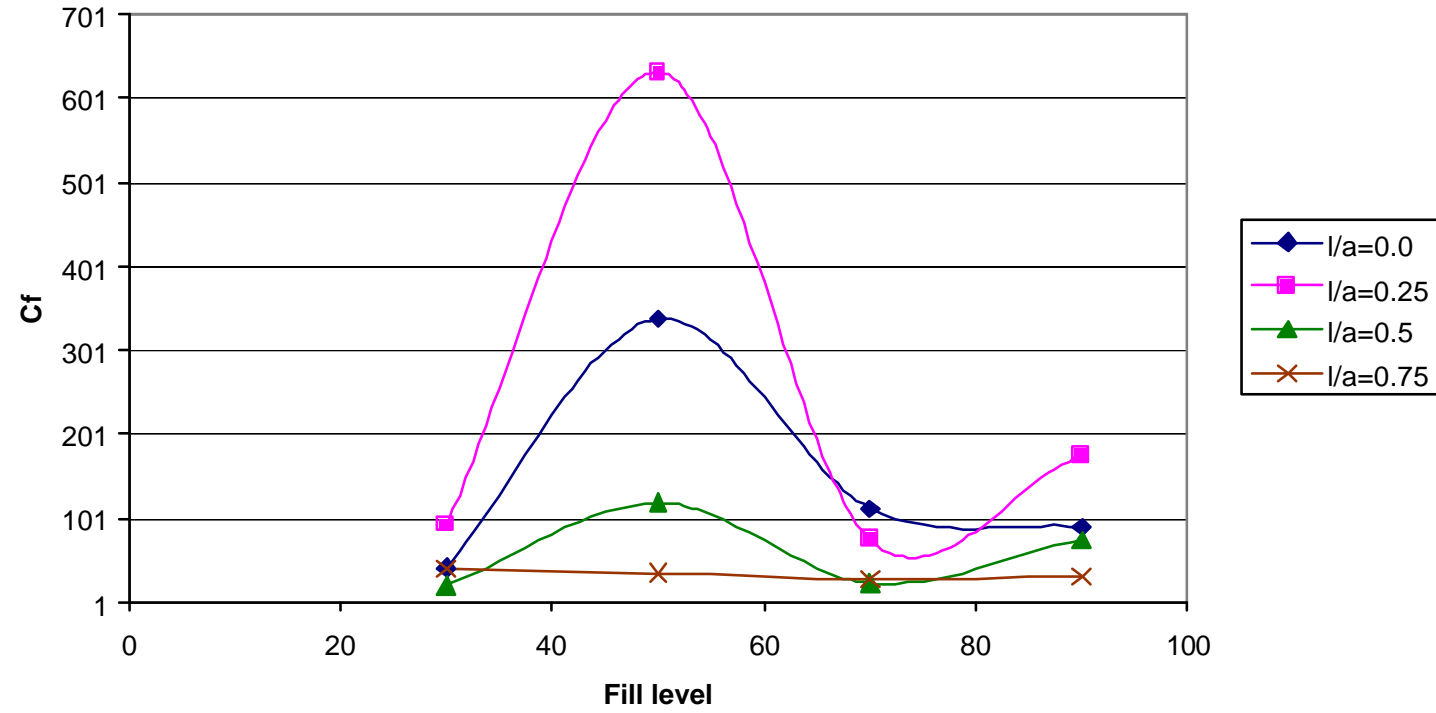

Figure $88 . \mathrm{C}_{\mathrm{f}}$ for elliptical tank with diagonal baffles in a TOP lane change maneuver

As shown in all the above results, diagonal baffles and horizontal-vertical baffles were successful in reducing peak moments and sloshing in most of the cases in a TOP lane. 


\section{CHAPTER 6}

\section{SUMMARY AND CONCLUSIONS}

\subsection{Summary}

In this study, attempts were made to determine the effect of kinematic viscosity of the fluid on damping of fluid sloshing while the tanker truck was undergoing different maneuvers. Attempts were also made to find out the effects of various baffles on damping the lateral sloshing in tankers maneuvering a constant radius turn and TOP lane change, and determine if the rollover threshold velocity would increase.

Computational fluid dynamics, models were developed using FLUENT with different baffles, fill levels and tank shapes. These models were first verified using results presented by Sankar et al. [16] and Salem [18]. These models were then used for all the analyses.

Analyses were run, using the models developed, to determine the effect of fluid kinematic viscosities on damping of the fluid sloshing. Five different liquids, with kinematic viscosities ranging from $7.0 \mathrm{E}-6 \mathrm{~kg} / \mathrm{m}-\mathrm{s}$ through $1.06 \mathrm{~kg} / \mathrm{m}$-s were chosen for this purpose. Circular and elliptical tanks were considered in this study. It was determined that kinematic viscosity had no effect on damping for the range of kinematic viscosity considered in this study. Thus, it was concluded that there was no necessity to include any torsional dampers in trammel pendulum models, if used to simulate the lateral sloshing of liquid in moving tankers. 
Four different baffle designs namely, horizontal baffles, vertical baffles, horizontal-vertical baffles, and diagonal baffles with three different lengths were studied to determine their effect on reducing the sloshing, and forces and moments on the tank walls. Two critical maneuvers, constant radius turn and double lane change maneuvers were investigated.

It was concluded that horizontal-vertical baffles yielded maximum reduction in the peak moments in a circular tank through a constant radius turn. Diagonal baffles yielded good results for fill levels of $30 \%$ and $70 \%$. A reduction of $59 \%$ for fill levels of $30 \%$ and $70 \%$ were observed with horizontal-vertical baffles. Reduction in the case of $50 \%$ fill level was $30 \%$. In the case of elliptical tank maneuvering a constant radius turn, diagonal baffles were found to be effective for fill levels of $30 \%$ and $70 \%$. Horizontalvertical baffles were found to be suitable for other fill levels.

In TOP lane change maneuver none of the baffles produced any significant reduction in peak moments. Diagonal baffles with an $1 / \mathrm{a}$ ratio of 0.75 were effective at reducing peak moments for lower fill levels, and vertical baffles with an 1/a ratio of 0.25 were effective for fill levels greater than $50 \%$ in a circular tank going through a TOP lane change maneuver. In case of an elliptical tank, configuration of horizontal-vertical baffles with an 1/a ratio of 0.75 was found to be the best at reducing the peak moments through a TOP lane change maneuver. 


\subsection{CONCLUSIONS}

1. Kinematic viscosity of the fluid has no effect on damping the fluid sloshing in partially filled heavy-duty tanker trucks.

2. Horizontal and vertical baffles are the best configuration for reducing the peak moments and sloshing when all the four fill levels (30\%, 50\%, 70\%, and 90\%), tank shapes (circular, and elliptical), and different maneuvers (constant radius, and TOP lane change) are considered.

3. Baffles can reduce the peak moments in partially filled heavy-duty tanker trucks by as much as $60 \%$.

4. Damping at the free surface (horizontal baffles in a $50 \%$ fill case) has a greater effect in reducing sloshing and peak moments than damping by any other configuration of baffles.

\subsection{FUTURE WORK}

Four configurations of baffles (horizontal, vertical, horizontal-vertical, and diagonal) were studied in this project. Horizontal baffles were very effective for $50 \%$ fill level and did not make much difference for fill levels higher than $50 \%$. It is quite evident from this that horizontal baffles just over the surface of the fluid or above the free surface are effective in reducing the sloshing and moments. To take care of different fill levels it is required to have horizontal baffles at more than one location (elevation). Baffles at different elevations will take care of different fill levels. This configuration of horizontal 
baffles has to be studied and optimized. In a similar way, vertical baffles also could be located at more than one position and reduce the fluid sloshing further. More than one pair of vertical baffles also has to be studied.

This study, being the first attempt in this direction, discussed the most basic configurations of baffles. Some other complex configurations of baffles such as movable baffles or baffles at an angle could be designed and studied.

Structural aspect of baffles needs to be studied. Nothing has been done in that regard in this research. Baffles discussed in this study will be supported at one end and behave like a cantilever. Chances of failure are more in this case because of the high bending moments at the end where the baffles will be supported. Ways to support the baffles strong enough have to be designed.

This is one of the very few studies towards reducing the lateral fluid sloshing inside heavy-duty tankers. Hopefully more intense research will be done in this regard and make transportation through tankers safer. 


\section{BIBLIOGRAPHY}

[1] SUMNER, IRVING E.; AND STOFAN, ANDREW J.: An Experimental Investigation of the Viscous Damping of Liquid Sloshing in Spherical Tanks. NASA TN D-1991, 1963.

[2] ABRAMSON, H. NORMAN; AND SILVERMAN, SANDOR: The Dynamic Behavior of Liquids in Moving Containers. NASA SP-106, 1966, pp. 105-140.

[3] SILVEIRA, MILTON A.; STEPHENS, DAVID G.; AND LEONARD, H. WAYNE: An Experimental Investigation of the Damping of Liquid Oscillations in Cylindrical Tanks With Various Baffles. NASA TN D-715, 1961.

[4] STOFAN, ANDREW J.; AND PAVLI, ALBERT J.: Experimental Damping of Liquid Oscillations in a Spherical Tank by Positive-Expulsion Bags and Diaphragms. NASA TN D-1311, 1962

[5] STOFAN, ANDREW J.; AND SUMNER, IRVING E.: Experimental Investigation of the Slosh-Damping Effectiveness of Positive-Expulsion Bags and Diaphragms in Spherical Tanks. NASA TN DN-1712, 1963.

[6] EULTZ, WERNER: A Can-Type Device Derived From Basic Slosh Studies. Rept. No. DSD-TR-4-58, Dev. Operations Div., ABMA, Red-stone Arsenal, Ala., Aug. 1958. Available from DDC, AD-203348.

[7] ABRAMSON, H. NORMAN; AND RANSLEBEN, GUIDO E., Jr.: Simulation of Fuel Sloshing Characteristics in Missile Tanks by use of Small Models. ARS J., vol. 30, no. 7, July 1960 , pp. 603-612. 
[8] ABRAMSON, H. NORMAN; AND RANSLEBEN, GUIDO E., Jr.: Some Studies of a Floating Lid Type Device for Suppression of Liquid Sloshing in rigid Cylindrical Tanks. TR No. 10, Contract DA-23-072-ORD-1251, Southwest Research Institute, May 1961.

[9] ABRAMSON, H. NORMAN; AND RANSLEBEN, GUIDO E., Jr.: Liquid Sloshing in Rigid Cylindrical Tanks Undergoing Pitching Motion. TR No. 11, Contract DA23-072-ORD-1251, Southwest Research Institute, May 1961.

[10] BAUER, HELMUT F.: Fluid Oscillations in the Containers of a Space Vehicle and Their Influence Upon Stability. NASA TR R-187, 1964.

[11] BAUER, HELMUT F.: On the Destabilizing effect of Liquids in Various Vehicles. Vehicle System Dynamics 1, 1972.

[12] STRANDBERG, LENNART: Lateral Stability of Road Tankers. National Swedish road and Traffic Research Institute, Sweden. Report No. 138A, 1978.

[13] LIDSTRÖM, MATS: Road Tankers' Overturning Limit - The Steady-State case (In Swedish). National Road and Traffic Research Institute, Sweden, Paper 1976-12-17, 1976.

[14] POPOV, G.; SANKAR, S.; AND SANKAR, T.S.: Dynamics of Liquid Sloshing in Baffled and Compartmented Road Containers. Journal of Fluids and Structures, 1993, 7, pp. 803-821.

[15] IBRAHIM, I.M.: Anti-slosh damper Design for Improving the Roll Dynamic Behavior of Cylindrical Tank Trucks. SAE 1999-01-3729.

[16] POPOV, G.; SANKAR, S.; AND SANKAR, T.S.: Dynamics of Liquid Sloshing in Elliptical Road Containers. Canadian Society of Mechanical Engineering, 1992. 
[17] ACQUARO, M.: Stability Analysis of Partially Filled Tanker Trucks Using a Finite Element Modeling Approach. Masters Thesis, West Virginia University, April 1999.

[18] Salem, I.M.: Rollover Stability of Partially Filled Heavy-Duty Elliptical Tankers Using Trammel Pendulums to Simulate Fluid Sloshing. Ph. D. Dissertation, West Virginia University, March 2000. 


\section{APPENDIX A}

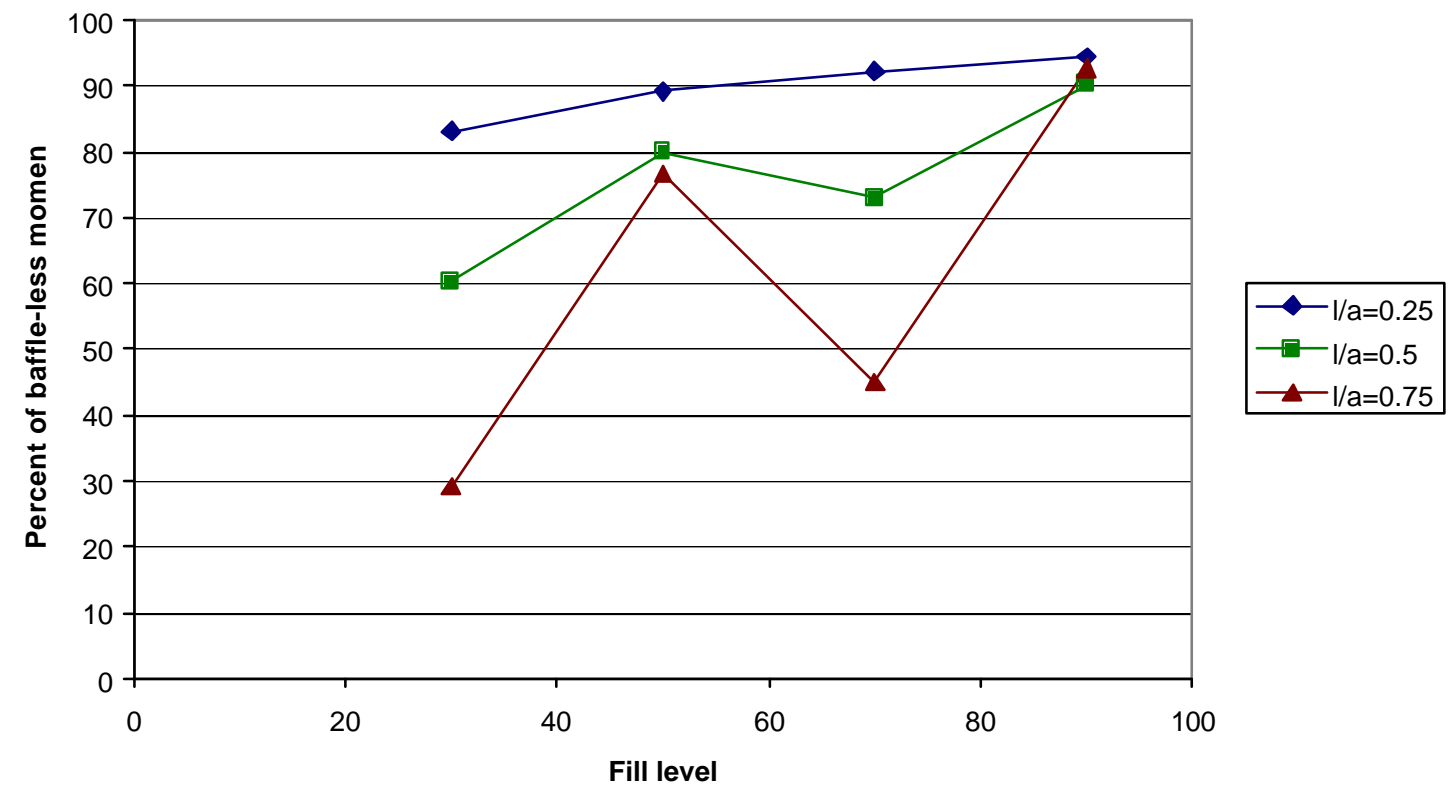

Figure 89. Percentage ratios of peak moments in an elliptical tank with diagonal baffles to peak moments without any baffles

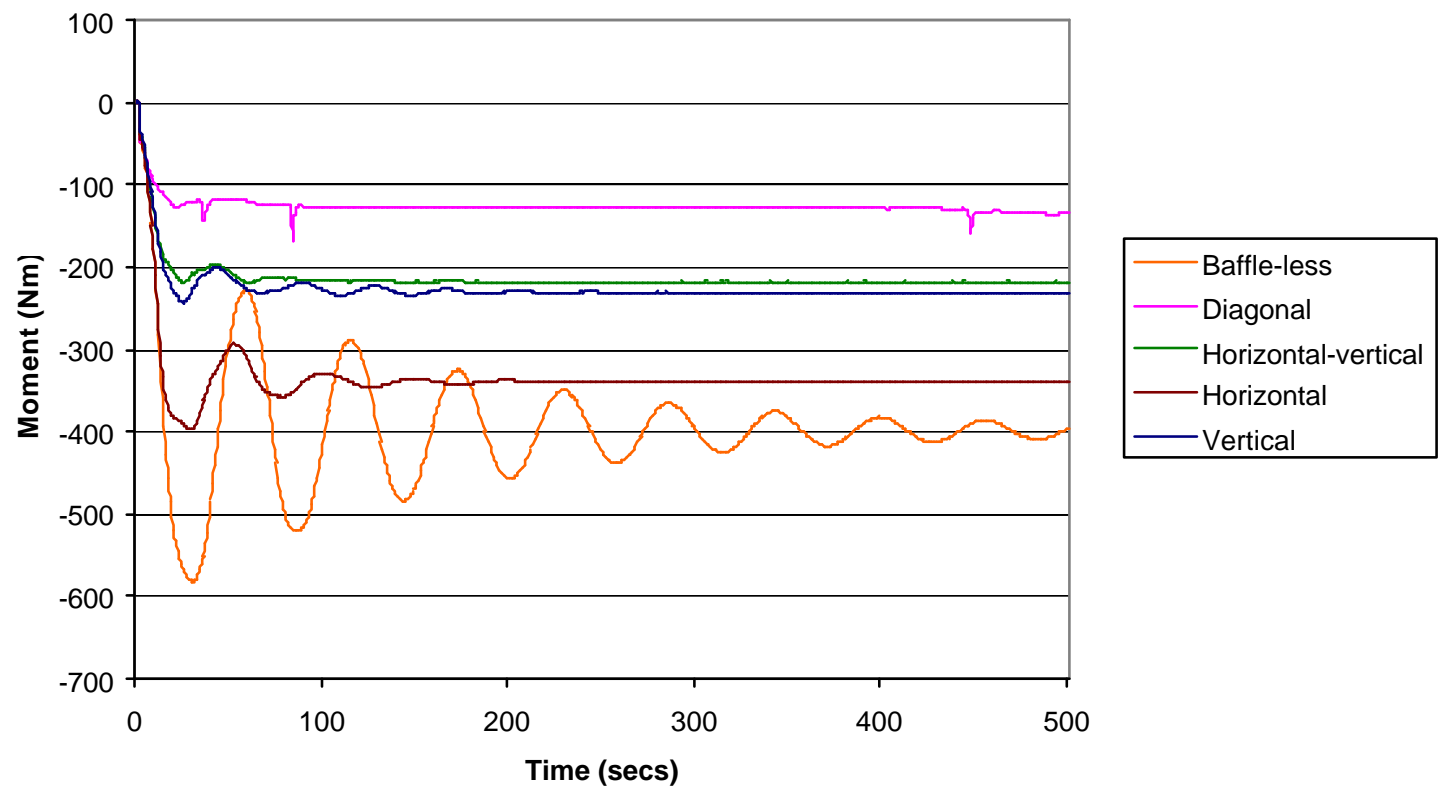

Figure 90. Moment with time for various baffles $(1 / \mathrm{a}=0.75)$ in an elliptical tank with $30 \%$ fill level 


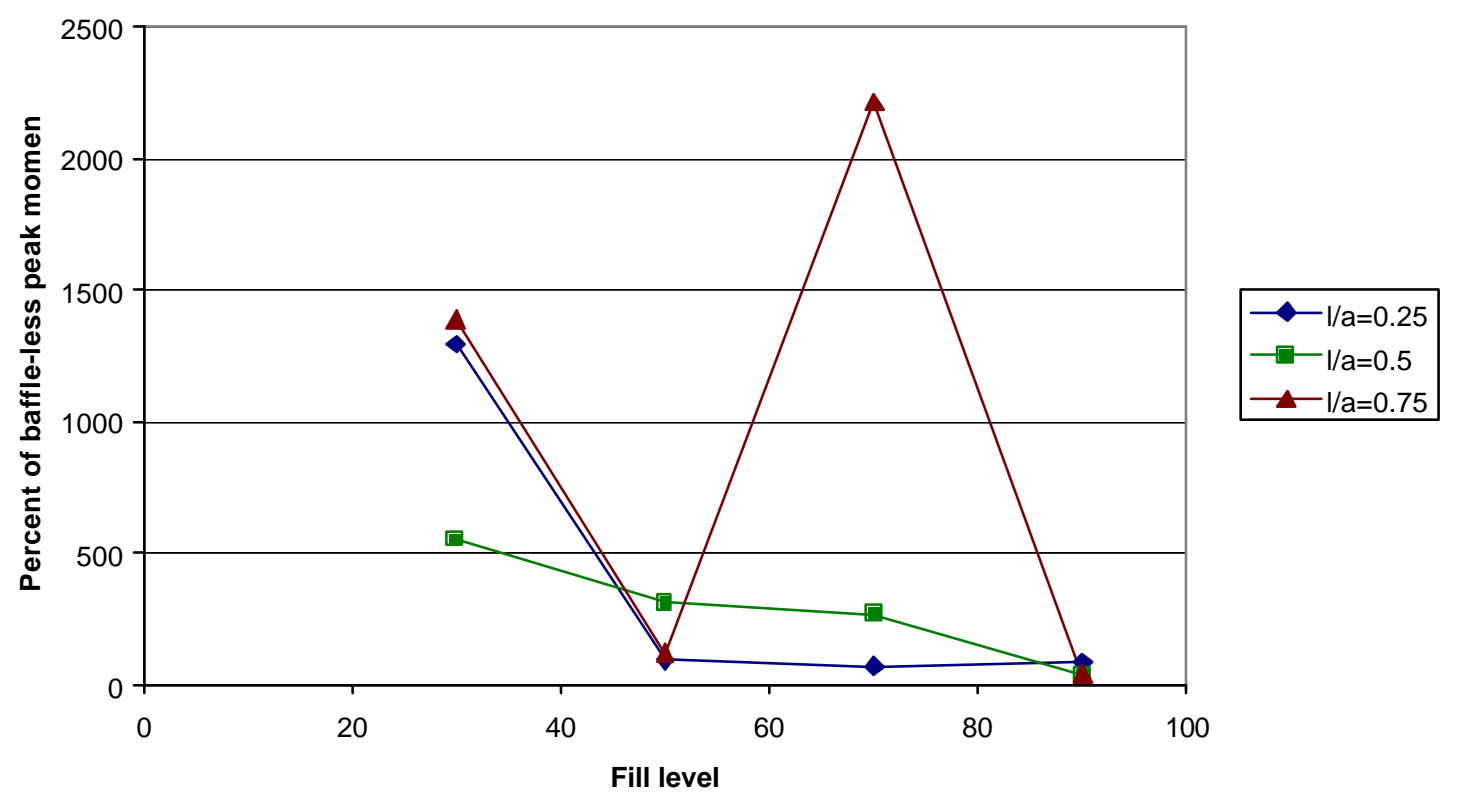

Figure 91. Percentage ratios of peak moments in a circular tank with horizontal baffles to peak moments without any baffles in TOP lane

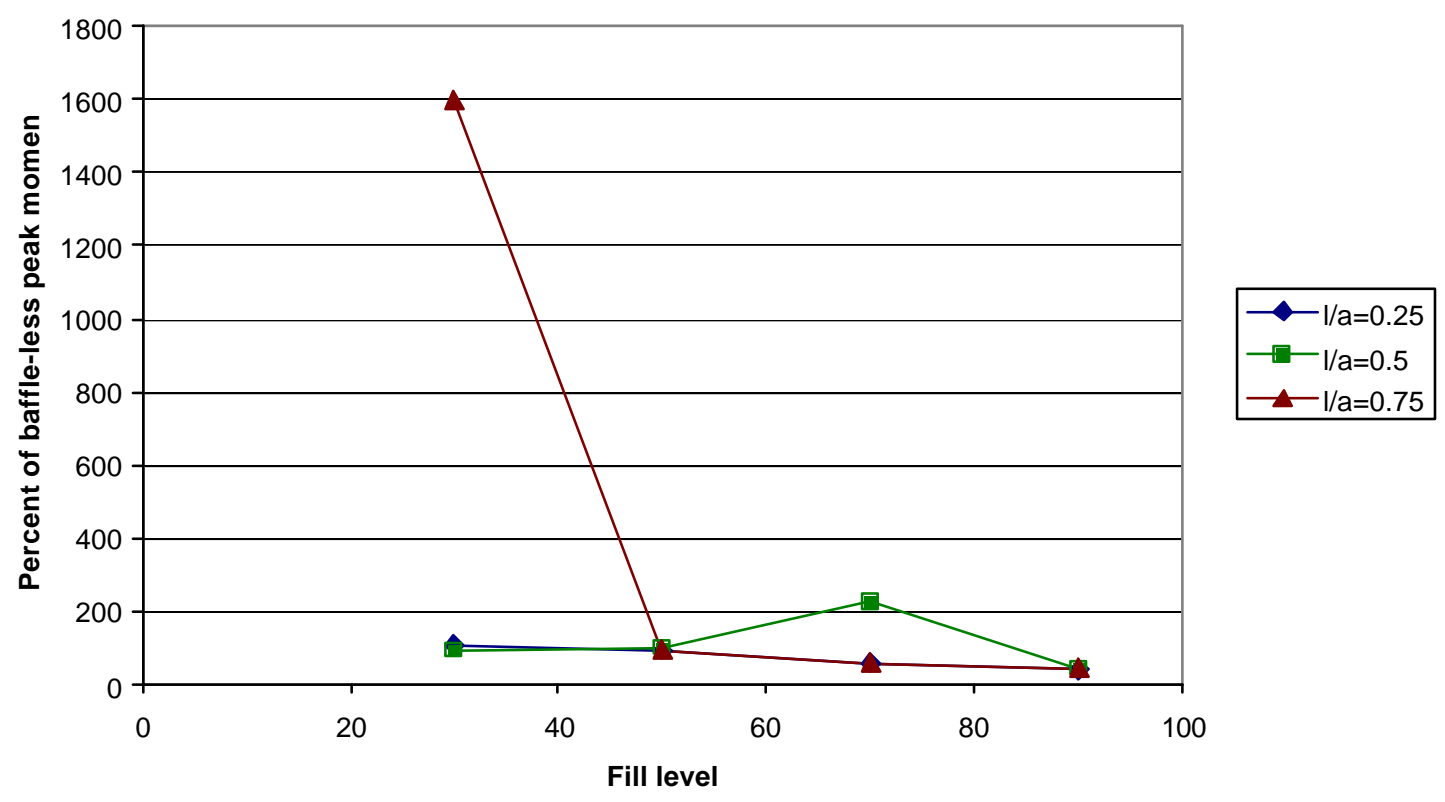

Figure 92. Percentage ratios of peak moments in a circular tank with vertical baffles to peak moments without any baffles in TOP lane 


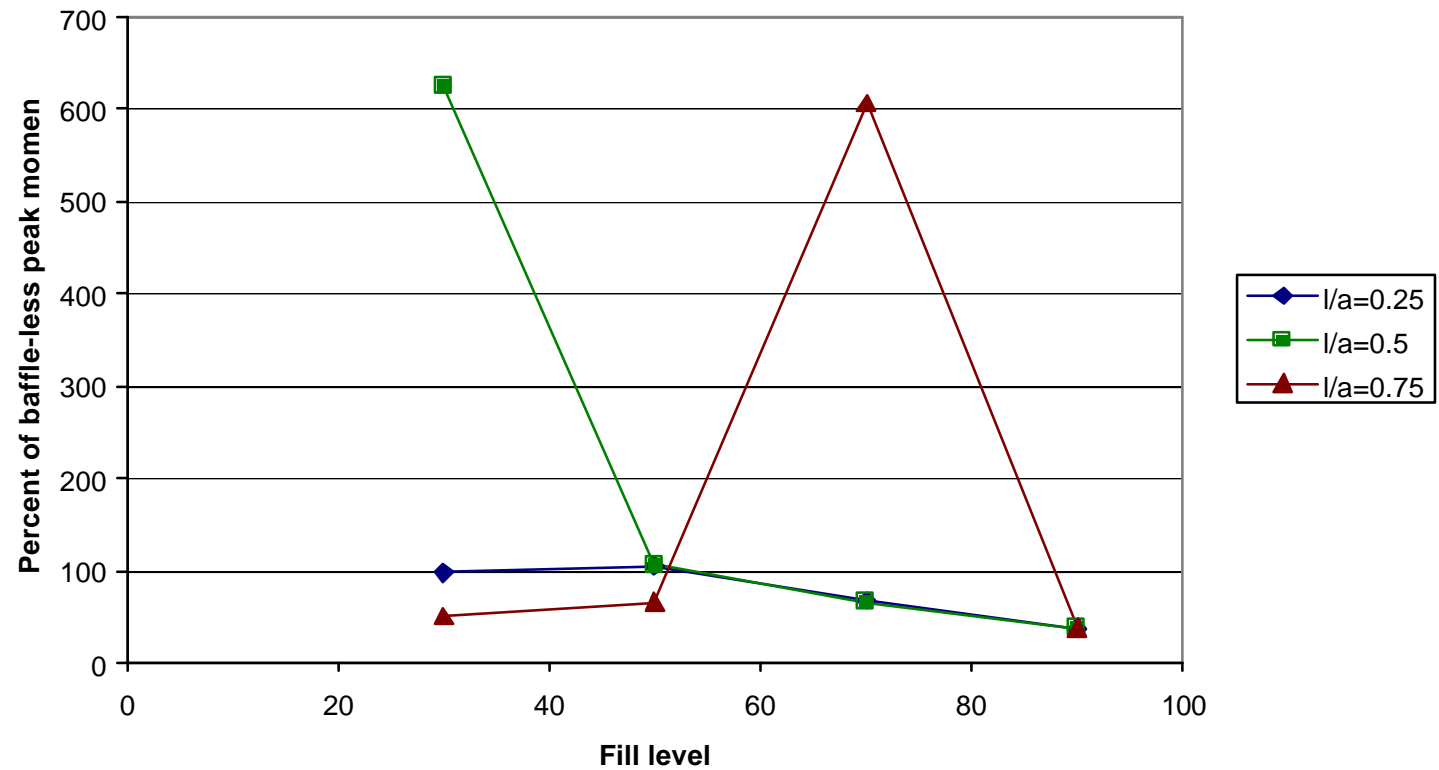

Figure 93. Percentage ratios of peak moments in a circular tank with horizontalvertical baffles to peak moments without any baffles in TOP lane
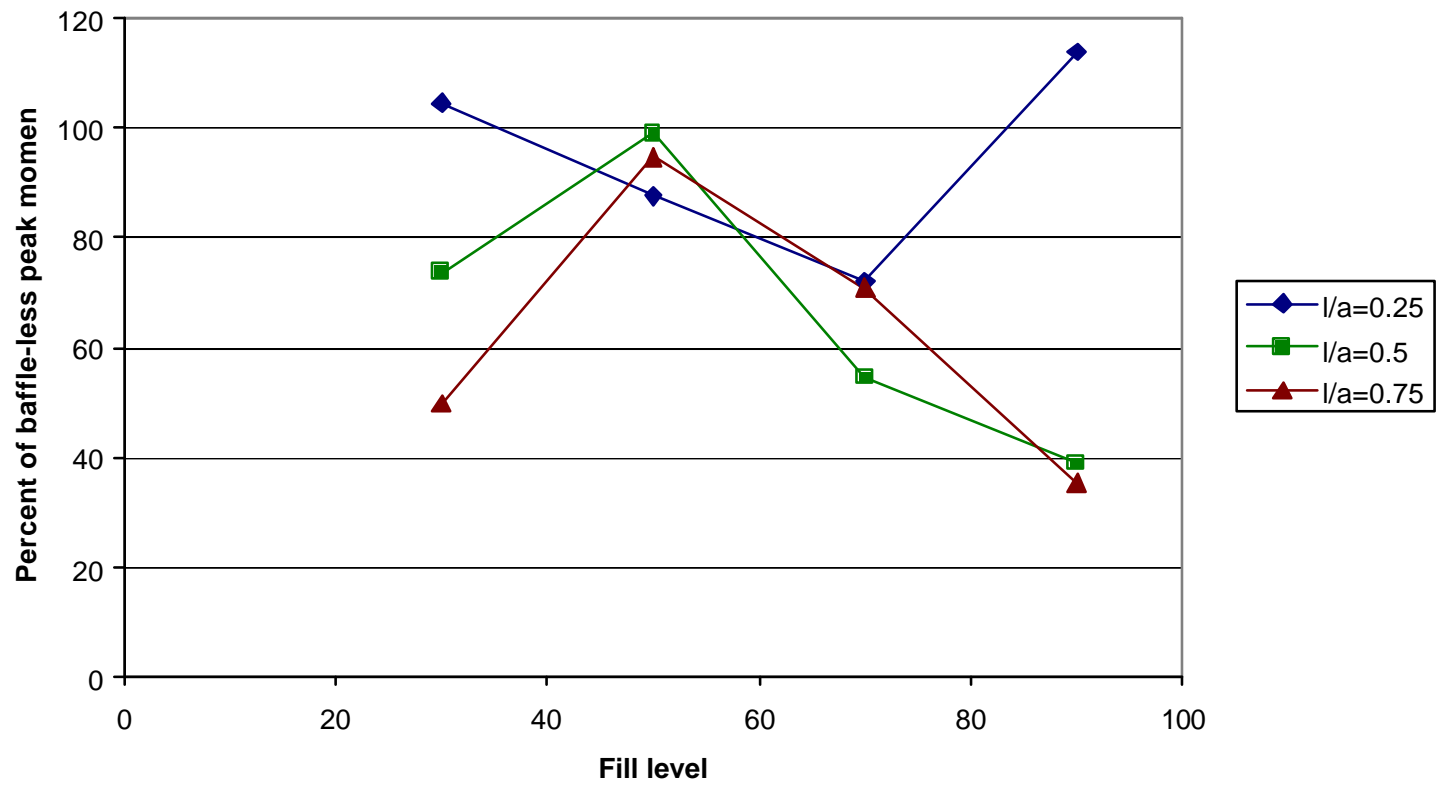

Figure 94. Percentage ratios of peak moments in a circular tank with diagonal baffles to peak moments without any baffles in TOP lane 


\section{APPENDIX B}

User Defined Sub-routine in ' $C$ ' to include time varying acceleration

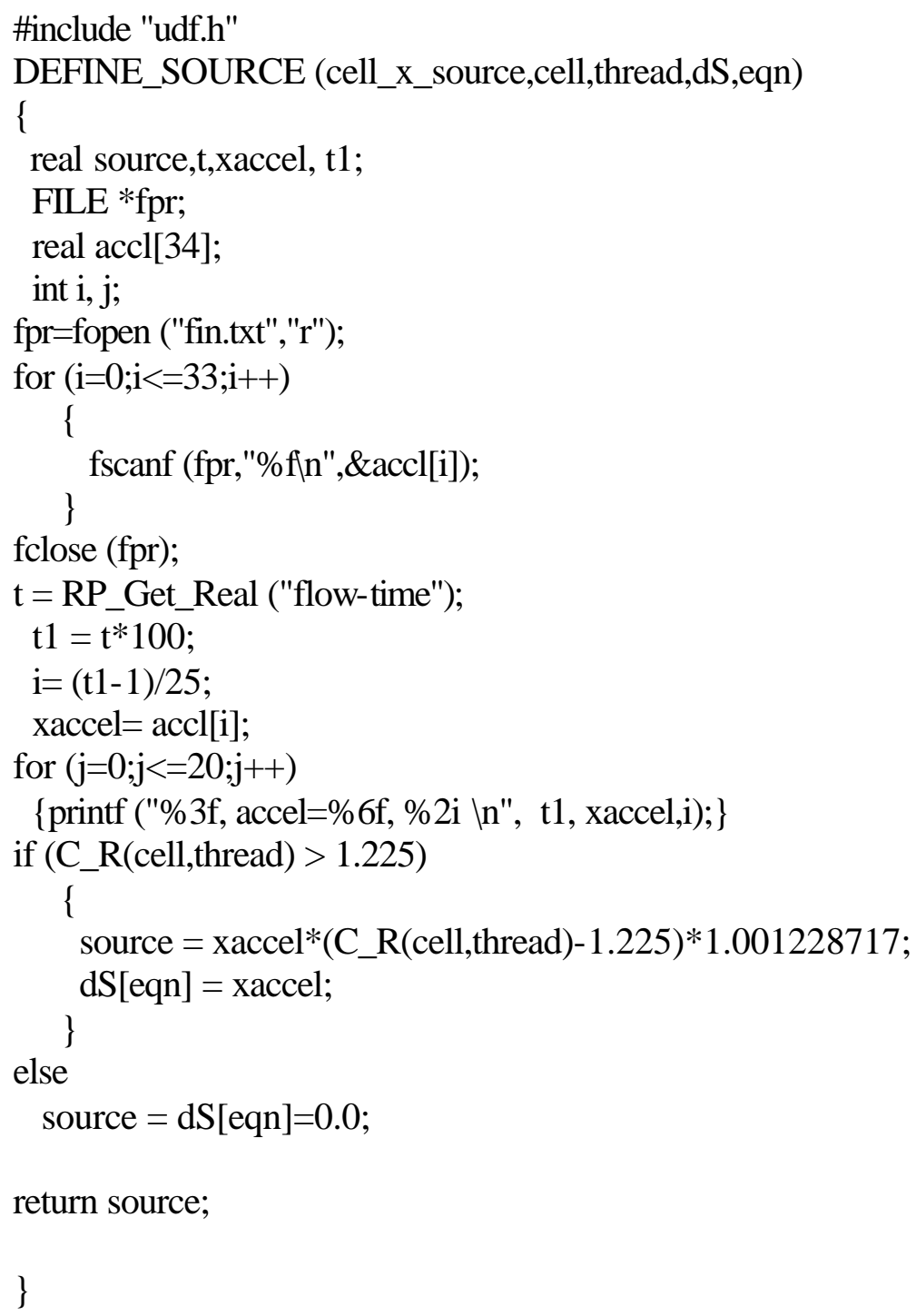


MATLAB Program to calculate the dynamic coefficients of moment and force from the files generated by FLUENT:

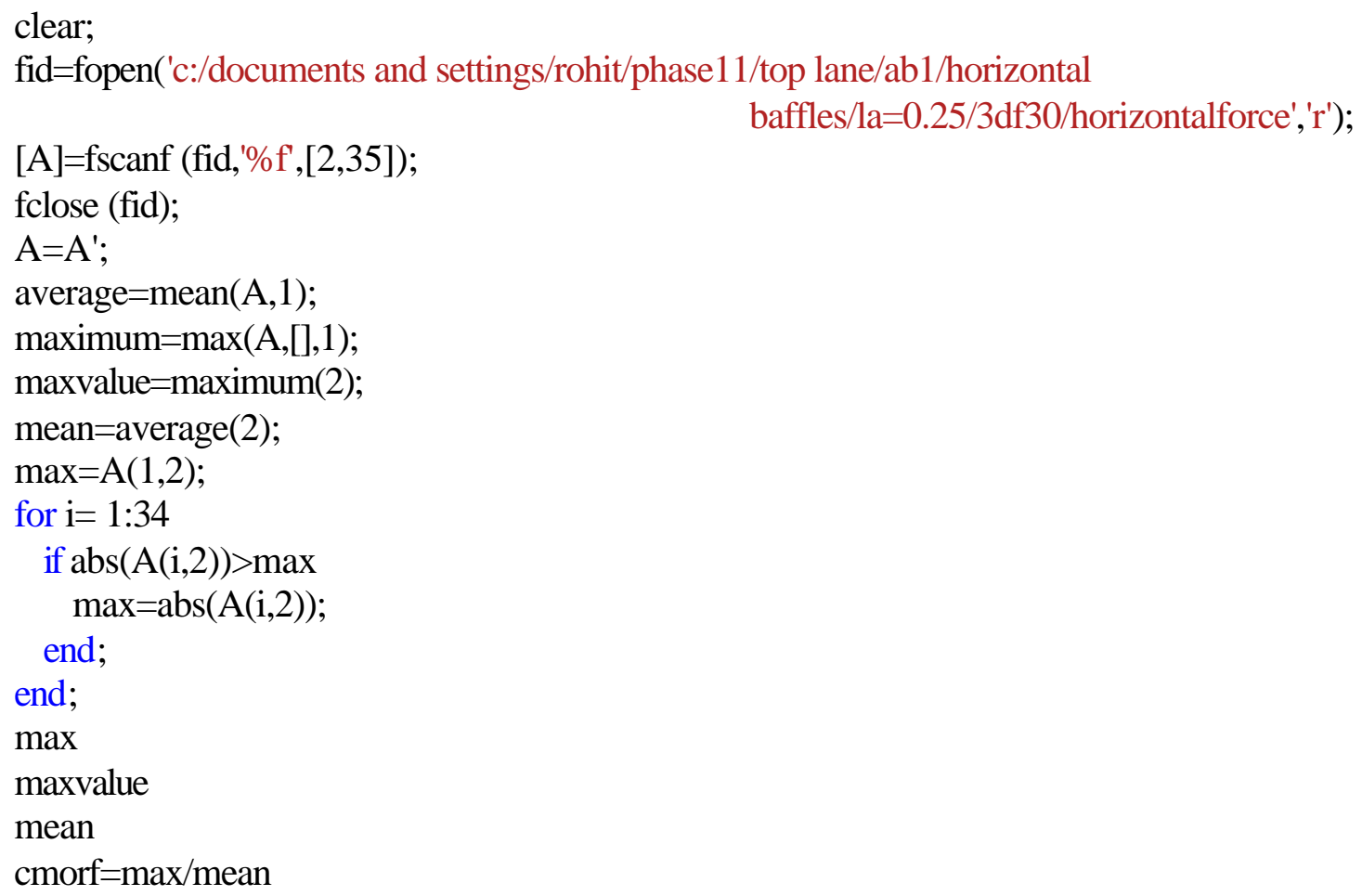

